\section{A) Check for updates}

Cite this: Inorg. Chem. Front., 2020, 7, 3805

\title{
Superactive NiFe-LDH/graphene nanocomposites as competent catalysts for water splitting reactions
}

\author{
Susanginee Nayak (D) * and Kulamani Parida (D) *
}

$\mathrm{NiFe}$-LDH has been recognized as the most efficient and cost-effective material for wider applications in electrocatalytic, photoelectrocatalytic, and photocatalytic water splitting, with supercapacitors and adsorbents, owing to their inimitable physicochemical properties. It is well known that standalone NiFe-LDH executes poor electrical conductivity, sluggish mass transfer, and low activity, which put a question mark on their catalytic efficiency and other applications that require superior electrical conductivity and exciton pair separation efficiency. Most importantly, this constraint creates a hindrance to their superior performance in the area of electrocatalytic and photochemical water splitting. To avoid these shortcomings, the coupled structure of NiFe-LDH/graphene has the potential to reflect properties of both NiFe-LDHs and conductive graphene, which completely overcome the shortcomings of counterparts, ensuring better performance and stability. This review aims to summarize the structural impact of NiFe-LDHs, with the interfacial role of graphene/graphene oxide (GO) by establishing a relationship between their structure and activity. Moreover, the emphasis has been laid on the latest development in NiFe-LDH/GO-based materials, along with attention to synthetic methods targeting the creation of a hierarchal porous nature in the materials with different growth approaches to NiFe-LDH on graphene for applications in electrocatalytic, photoelectrocatalytic, and photocatalytic water splitting activities. The latest research and development in this field using NiFe-LDH/graphene with a sensible intermixing of active sites and conductive framework is explored.

Received 16th June 2020 Accepted 19th August 2020

DOI: 10.1039/d0qi00700e rsc.li/frontiers-inorganic and is a green technology that can combat all these energy hurdles with minimal greenhouse gas emissions. ${ }^{4,5}$ As a product of water splitting, natural $\mathrm{H}_{2}$ and $\mathrm{O}_{2}$ gas can be produced from $\mathrm{H}_{2} \mathrm{O}$ by the electrocatalytic (EC) method using lowcost electricity, or the photoelectrochemical (PEC) method, or the photocatalytic (PC) method, which might provide the effective use of solar energy. ${ }^{6-8}$ The EC and PEC water splitting in modern-era electrolyzers are versatile methods for the essential manufacture of green and clean fuels by using sustainable energy resources. In these electrolyzers, the half-cell reaction at the anode is the oxygen evolution reaction (OER, $2 \mathrm{H}_{2} \mathrm{O} \rightarrow \mathrm{O}_{2} \uparrow+4 \mathrm{H}^{+}+4 \mathrm{e}^{-}$) and the product $\mathrm{O}_{2}$ gas, is unconfined. ${ }^{9,10}$ However, extensive overpotential is required in the OER to overcome its sluggish reaction kinetics. ${ }^{11}$ Similarly, the cathodic half-reaction is the hydrogen evolution reaction (HER, $2 \mathrm{H}^{+}+2 \mathrm{e}^{-} \rightarrow \mathrm{H}_{2}$ ). In the overall water splitting of $\mathrm{H}_{2} \mathrm{O}$ into $\mathrm{H}_{2}$ and $\mathrm{O}_{2}$, gas crossover may be possible in the long run, which raises the issue of safety along with a decrease in energy conversion efficiency due to the back reaction to $\mathrm{H}_{2} \mathrm{O}$ in acidic, or $\mathrm{OH}^{-}$ions in alkaline media. ${ }^{12} \mathrm{~A}$ key objective of a good electrocatalyst for electrochemical reactions depends on (i) good electrically active sites for conductivity, (ii) surface area, (iii)
Centre for Nano Science and Nano Technology, Siksha 'O' Anusandhan Deemed to be University, Bhubaneswar-751030, Odisha, India.E-mail: susanginee@gmail.com, kulamaniparida@soauniversity.ac.in,paridakulamani@yahoo.com;

Fax: +91-674-2350642; Tel: +91-674-2351777 
gas adsorption sites, and (iv) stability. ${ }^{13}$ PEC research strives for the advancement of novel and efficient photoelectrodes. In PEC, solitary photoelectrodes are being utilized for both water electrolysis and solar energy conversion; the photocathode (p-type semiconductor) or photoanode (n-type semiconductor) or both electrodes involve several reaction steps such as (i) the ability to exceptionally harvest light, (ii) easy charge separation and transfer, and (iii) fast OER kinetics. ${ }^{14}$ In PEC, band bending occurs at the interface of both electrodes and the electrolyte, which is the dynamic of the force responsible for the separation and transfer of charge carriers. The photoinduced holes transported at the photoanode are responsible for the OER, and electrons at the surface of the photocathode are responsible for the HER. In a heterogeneous photocatalytic water splitting process, photocatalysts are used in powder form and dispersed in polar solvents, and this simple method is more suitable than the PEC method as the economy and reaction conditions using external bias are the main prerequisites in a PEC reaction. ${ }^{15,16}$ The photocatalytic route or lightdriven water splitting to generate molecular $\mathrm{H}_{2}$ and $\mathrm{O}_{2}$ using a powdered photocatalyst has tremendous potential for the conversion of solar energy to chemical energy. Under the irradiation of light energy greater than or equal to the energy of the bandgap barrier of a semiconductor, electronic excitation takes place from the valence band (VB) to the conduction band (CB) and leaves behind holes in the VB. The CB electrons can reduce protons to produce $\mathrm{H}_{2}$, whereas the holes produced in the VB can oxidize $\mathrm{H}_{2} \mathrm{O}$ to generate $\mathrm{O}_{2}$ gas. ${ }^{17}$ An efficient photocatalytic semiconductor mainly depends upon three factors: (i) light absorption capability, (ii) separation and movement of photoinduced charges, (iii) reduction and oxidation reactions occurring on the surface of a catalyst. ${ }^{18}$ An inclusive deliberation of the above is the input for addressing the confinement of emerging materials and related energy issues.

Achieving superactive catalysts possessing all these characteristics of high-density active sites with energy generation $\left(\mathrm{H}_{2}\right.$ and $\mathrm{O}_{2}$ ) by the electrochemical, photochemical or photocatalytic route is a critical research area, and a variety of noblemetal and noble-metal-free catalysts have been explored to fulfill these requirements. ${ }^{9,15} \mathrm{Pt}$ is the best catalyst used for HER but its limitations include stability-related issues and high cost. Similarly, Au and Pd have also been used in electrocatalytic $\mathrm{HER}$, photo/electrocatalytic $\mathrm{H}_{2}$ evolution applications. Noble metal oxides like $\mathrm{RuO}_{2}$ and $\mathrm{IrO}_{2}$ are established as exceptionally active OER electrocatalysts both in alkaline and acidic media. However, their high cost and limited resources hinder their industrial applications. To overcome these limitations, much attention has been devoted to transition metal (TM) phosphides ${ }^{19}$ nitrides, ${ }^{20}$ and sulfides ${ }^{21}$ but these oxygenfamily-based TMs possess some drawbacks i.e., corrosion and instability. It is therefore an urgent task to develop catalysts made up of earth-abundant elements of low price, high stability and high efficiency. Recently, layered double hydroxides

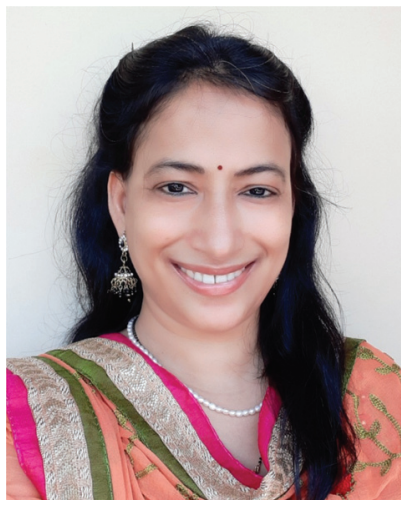

Susanginee Nayak
Dr Susanginee Nayak completed her Master of Science (M.Sc.) in 2004 at Sambalpur University, Odisha, India, and then obtained an M.Phil in Chemistry in 2009, from the University of Delhi. She worked as a Junior Research Fellow at ANDC College, University of Delhi, and Senior Project Fellow at CSIR-IMMT, Bhubaneswar. She then completed her Ph.D. in 2019 at Siksha 'O' Anusandhan (Deemed to be University) under the guidance of Prof. Kulamani Parida. She is currently working as a Post-Doctoral Research fellow at the Centre for Nano Science and Nano Technology (CNSNT), Dept. of Chemistry, Institute of Technical Education and Research, Siksha ' $O$ ' Anusandhan (Deemed to be University). She is the author of 10 international journal articles, with 1 review article and has filed three patents. Her research activities are mainly focused on the synthesis of modified layered double hydroxides (LDHs) based nanocomposite materials towards energy conversion and environmental organic pollutant removal.

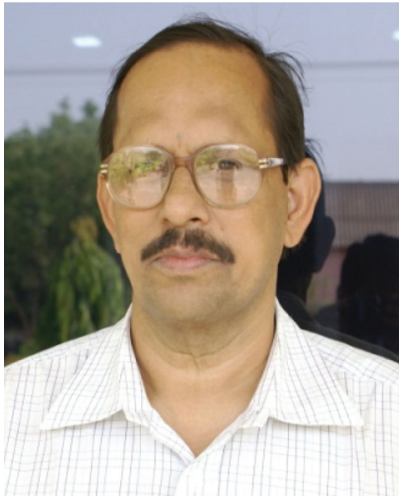

Kulamani Parida
Prof. Kulamani Parida is a distinguished Professor in Chemistry and the Director of the Centre for Nano Science and Nano Technology, Siksha O Anusandhan, Deemed to be University, Bhubaneswar, Odisha, India. Before joining at SOA, Deemed to be University, he worked 37 years at CSIR-IMMT, Bhubaneswar in different capacities and retired from the position of Chief Scientist and Head of Colloids and Materials Chemistry. Dr. Parida has made significant contributions toward exploring the chemistry related to structural, morphological, optical, electronic and electrochemical properties of a number of wide bandgap and narrow bandgap semiconductors, doped metal oxides, metal carbides, metal sulphides and nitrides for the effective visible light absorption and charge separation required for efficient photocatalytic water splitting, degradation of pollutants and synthesis of fine chemicals. He has published around 400 papers in high impact factor journals, 7 book chapters, filed 35 patents and supervised 50 Ph.D. students. The important and current relevance of his work is amply reflected in the large number of citations ( 18000$)$ of his work and the H index of 72. 
(LDHs) containing 1st-row earth-abundant TMs (Ni, Fe, Co, $\mathrm{Mn}$, etc.) have been identified for catalyzing the alkaline OER. ${ }^{22}$ LDHs involve the isomorphous substitution of a divalent metal cation by a trivalent metal cation, forming brucitelike host layers of $\mathrm{Mg}(\mathrm{OH})_{2}$. The metal cations are octahedrally coordinated by hydroxyl groups forming $\mathrm{MO}_{6}$ octahedra, where vertexes of the $\mathrm{O}-\mathrm{H}$ groups are perpendicular to the plane of the host layers that connect to form infinite $2 \mathrm{D}$ sheets. LDHs can accommodate charge-compensating organic or inorganic anions and water molecules inside the interlayer in order to compensate for the excess positive charge developed due to the isomorphous substitution. ${ }^{23}$ LDHs were first discovered in the form of mineral hydrotalcite as $\mathrm{Mg}_{6} \mathrm{Al}_{2}\left(\mathrm{CO}_{3}\right)$ $(\mathrm{OH})_{16} \cdot 4\left(\mathrm{H}_{2} \mathrm{O}\right)$, where the $\mathrm{Al}^{3+}$ is substituted for $\mathrm{Mg}^{2+}$ in brucite-like layers of $\mathrm{Mg}(\mathrm{OH})_{2}$, with $\mathrm{CO}_{3}{ }^{2-}$ and water in the interlayer. ${ }^{24-26}$ The chemical formula of $\mathrm{LDH}$ is usually represented as $\left[\mathrm{M}(\mathrm{II})_{1-x} \mathrm{M}(\mathrm{III})_{x}(\mathrm{OH})_{2}\right]^{x+}\left[\mathrm{A}^{n-}{ }_{x / n} \cdot m \mathrm{H}_{2} \mathrm{O}\right]^{x-}$, where $\mathrm{M}$ (II) is divalent and $\mathrm{M}$ (III) is a trivalent metal cation. ' $\mathrm{A}$ ' is the interlayer organic or inorganic anion that compensates for the excessive increase of positive charge in the brucite-like layers. ' $n$ ' is the charge on the interlayer anion, $m$ represents the amount of water, ${ }^{27,28} x$ is the molar ratio of the trivalent cation, and the density of the surface charge is proportional to the ratio of the trivalent metal cations, $x=\mathrm{M}(\mathrm{III}) /[\mathrm{M}(\mathrm{II})+\mathrm{M}(\mathrm{III})]$. Generally, the compositional range of LDHs resides within 0.2 $\leq x \leq 0.33$. The wide variety of metal cations, metallic ratios $\mathrm{M}(\mathrm{II}) / \mathrm{M}$ (III), and anions associated with $\mathrm{LDH}$ are easily exchangeable and create flexibility in the electronic structure and composition. ${ }^{23}$ On the atomic scale, diverse metal cations can be used to build stable LDHs; for example: $\mathrm{Fe}^{2+}, \mathrm{Mg}^{2+}$, $\mathrm{Ni}^{2+}, \mathrm{Co}^{2+}, \mathrm{Cu}^{2+}, \mathrm{Mn}^{2+}, \mathrm{Zn}^{2+}, \mathrm{Cd}^{2+}, \mathrm{Pd}^{2+}$ and $\mathrm{Ca}^{2+}$ are divalent cations and $\mathrm{Co}^{3+}, \mathrm{Al}^{3+}, \mathrm{Mn}^{3+}, \mathrm{Fe}^{3+}, \mathrm{Cr}^{3+}, \mathrm{Ga}^{3+}, \mathrm{V}^{3+}$ and $\mathrm{Tb}^{3+}$ are trivalent cations. Tetravalent cations, such as $\mathrm{Ti}^{4+}(0.61 \AA), \mathrm{Zr}^{4+}$ $(0.72 \AA)$ and $\mathrm{Sn}^{4+}(0.69 \AA)$ can be used to build stable LDHs. The most unique property of $\mathrm{LDH}$ is that on heat treatment, their hexagonal phase structure is destroyed and they form the mixed metal oxide phase. However, the original LDH structure can be reconstructed by the hydration process of the mixed metal oxide, and these properties are known as the structural memory effect. Additionally, the bulk manufacture of LDHs can be achieved by applying the latest synthetic strategy, followed by their applications in the areas of catalysis, photocatalysis, adsorbents, photochemistry, biomedical science, electrochemistry, magnetisation, and polymerisation. ${ }^{27-29,30}$ The authors, along with other groups, have demonstrated the potential of various pristine or modified binary and ternary LDHs, cation and anion-doped LDH and composites of LDH towards superior energy conversion or energy storage applications. These include using NiFe-LDH, ${ }^{27,31-34} \mathrm{MgAl}-\mathrm{LDH},{ }^{28}$ MgCr-LDH, ${ }^{35}$ ZnCr-LDH, ${ }^{36-38}$ CoFe-LDH, ${ }^{39,40}$ NiLa-LDH, ${ }^{41}$ NiMn-LDH, ${ }^{42}$ CoMn-LDH, ${ }^{43}$ S-doped NiCoFe-LDH, ${ }^{44} \mathrm{Ni} / \mathrm{ZnCr}$ $\mathrm{LDH}^{45}{ }^{45} \mathrm{MgAlFe}-\mathrm{LDH},{ }^{46}$ etc. Specifically, the authors recently reported various NiFe-LDH-based heterostructured materials for excellent PC water splitting activities. ${ }^{27,33,34}$

In the early years, $\mathrm{Ni}$ and $\mathrm{Ni}$-based oxides were mostly engaged in the OER under basic media. ${ }^{47,48}$ Subsequently, combinations of $\mathrm{Ni}$ with $\mathrm{Fe}$ as impurities in $\mathrm{Ni}(\mathrm{OH})_{2}$ were noted to lower the overpotential of OER in Ni-based alkaline batteries. ${ }^{49-51}$ The incorporation of Fe impurities as $0.01 \%$ in NiFe has a strong effect in lowering the overpotential of the OER. ${ }^{49-51}$ Generally, NiFe-based materials such as NiFe alloys, ${ }^{52-54}$ oxides, ${ }^{54}$ and (oxy) hydroxides, ${ }^{54-56}$ are used as earth-abundant catalysts that are effective for the OER under basic media. Active surface areas containing copious amounts of active sites are among the major strategies for fabricating OER electrocatalysts, whereas, in this race, the morphological alteration of NiFe electrocatalysts could fulfill these strategies. Among the TM-based LDHs, NiFe-LDHs is the best-studied material owing to its unique lamellar structures, tunable compositions, easy intercalation, cost-effectiveness, low toxicity, and eco-friendly nature. ${ }^{57} \mathrm{~A}$ recent study of the OER performances of Mn, Fe, Co and Ni-based (oxy) hydroxides, revealed that the corresponding catalytic performances depend on the $\mathrm{OH}-\mathrm{M}^{2+\delta}$ bond strength of the order $\mathrm{Ni}<\mathrm{Co}<\mathrm{Fe}<\mathrm{Mn} .{ }^{58}$ As a result, extremely strong or weak $\mathrm{M}-\mathrm{OH}$ bonds can slow down the OER activity. Similarly, a first-principles study revealed that $\mathrm{O}_{2}$ on NiFe-LDHs can be easily generated from $\mathrm{OOH}^{*}$ species and the $\mathrm{Fe}(\mathrm{OH})_{3}$ sites are potentially more active than the $\mathrm{Ni}$ $(\mathrm{OH})_{2}$ sites. In this aspect, NiFe-LDHs possess inherent catalytic activity towards electrochemical and photoelectrochemical OER in comparison to other TM and noble metal catalysts such as $\mathrm{IrO}_{2}$ and $\mathrm{RuO}_{2} \cdot{ }^{33,34,59-61}$ From the perspective of a photocatalyst, NiFe-LDH possesses a suitable bandgap (2.2 $\mathrm{eV}$ ), band edge potentials ( $\mathrm{CB}=-0.01 \mathrm{~V}, \mathrm{VB}=+2.19 \mathrm{~V} v s$. NHE) and intrinsic absorption properties of electronic charge transitions through excited inter-and intra-electronic paths, such as oxo-bridged $\left(\mathrm{Ni}^{2+}-\mathrm{O}-\mathrm{Fe}^{3+}\right), \mathrm{d}-\mathrm{d}$ transitions in $\mathrm{Ni}^{2+}$ cations and ligand to metal charge transfer (LMCT) in $\mathrm{O} \rightarrow \mathrm{Ni}^{2+} / \mathrm{Fe}^{3+}$, which are suitable for PC water splitting activities. ${ }^{27,33,34,62}$ However, the conventional NiFe-LDH with bulky structure suffers from meager photo/electrical conductivity and mass transfer due to deficient active sites and electronic charge transport, and solubility product differences among the metal precursors $\left(\left(\mathrm{Ni}(\mathrm{OH})_{2}, K_{\mathrm{sp}}=5.5 \times 10^{-16}\right.\right.$ and $\mathrm{Fe}(\mathrm{OH})_{3}, K_{\mathrm{sp}}=2.8 \times$ $\left.10^{-39}\right)$. Optimal parameters for controlling the structure and composition of LDHs are vital factors for electrochemical and photocatalytic applications..$^{33,34,59,60,63,64}$ Yin et al. reported NiFe-LDH/C that displayed superior OER performances. ${ }^{65}$ These NiFe-LDH/C-coupled systems displayed higher binding energies for $\mathrm{Ni} 2 \mathrm{p}$ and Fe $2 \mathrm{p}$ as compared to NiFe-LDH, which was confirmed by the inflections in the local electronic environments of $\mathrm{Ni}$ and Fe cations. ${ }^{65}$ The coupling reaction between carbon and NiFe-LDH caused distortions in the electronic environment and formed the $\mathrm{Ni} / \mathrm{Fe}-\mathrm{O}-\mathrm{C}$ bond, which resulted in a decrease in the crystal size of $\mathrm{NiFe}-\mathrm{LDH}$ in the NiFe-LDH/C system. ${ }^{66}$ Hence, conductive substrates or stabilizers like layered carbon materials, such as graphene, could be united to activate the active sites of NiFe-LDH and are together capable of overall water splitting efficiency. ${ }^{67-69}$

Graphene is packed with carbon monolayers that are arranged atomically in a honeycomb-like structure with superior electron mobility, high conductivity, and an elevated 
specific surface area. The conductive carbon lattice of graphene is the anchoring site for nanomaterials to form a variety of functional materials. Importantly, the graphene-based materials, for example, GO, possess unique physicochemical properties and have been widely used in adsorption, electrocatalysis, photoelectrocatalysis and photocatalysis. $^{70,71}$ In addition to the excellent conductivity and large surface area of $\sim 2630 \mathrm{~m}^{2} \mathrm{~g}^{-1}$, the GO matrix contains oxygen functionalities such as hydroxyl, epoxide and carboxylic groups, which can form epoxide-assisted metal oxide networks with NiFe-LDH under ambient conditions. Alternatively, the rich density of the $\mathrm{sp}^{2}$ bonded carbon present in rGO is anticipated to maintain the stability of the coupled catalytic system under sluggish OER reactions. The strong metal-rGO bonding interactions, in terms of the overlapping of the dsp and $\mathrm{sp}^{2}$ orbitals of rGO with $\mathrm{Ni}(\mathrm{OH})_{2}$ and the $\mathrm{Fe}(\mathrm{OH})_{3}$ layer of NiFe-LDH, are responsible for the photocatalytic and electrocatalytic activities. ${ }^{72,73}$ Similarly, NiFe-LDH in combination with heteroatom-doped graphene, ${ }^{74}$ and graphene hydrogel ${ }^{75}$ were reported to exhibit superior water splitting performances. Yet, heteroatom-doped graphene is advantageous because of its chemistry containing defective sites for binding the transition metal cations of LDH. $^{74}$ Hence, NiFe-LDH/graphene could provide equilibration between current collection and the active sites, even altering the electronic environment of NiFe-LDH and, on the whole, increase the kinetics of the charge transfer path. ${ }^{65,67}$

In general, two-dimensional (2D) nanomaterials give rise to a larger specific surface area and, due to forced dimensional orientation, the electron or charge or ion-pair movement is smoother as compared to bulk materials, and is responsible for the superior electrocatalytic and photocatalytic processes. Motivated along these lines, there are various advanced techniques such as solvothermal/hydrothermal, spin-coating, electrodeposition, ion-exchange, and microwave-assisted synthetic approaches that have been identified for the successful preparation of NiFe-LDH/2D-graphene. ${ }^{13}$ In most cases, NiFe-LDH/ 2D-graphene can be prepared by a one-step spontaneous coprecipitation method, in which the oxidation of the divalent metal cations to trivalent or higher valence takes place due to the presence of air or $\mathrm{O}_{2}$ dissolved in solution. A detailed discussion of the synthetic strategy of NiFe-LDH/2D-graphene is provided in the Preparation section below. Moreover, the 3D graphene or GO structure possesses a more axial orientation as compared to the $2 \mathrm{D}$ graphene structure, and properties like hierarchical porosity with entangled structure, multidirectional electron mobility, and enhanced conductivity, benefit superior catalytic performances. ${ }^{76}$ The hierarchical porous structure of $3 \mathrm{D}$ graphene is a potential substrate that can be jammed with large quantities of catalytically active sites. The combination of NiFe-LDH with 3D graphene (NiFe-LDH/3D graphene) provides a larger surface area, accompanied by electrical conductivity and a hierarchical porous structure for detecting the diffusion of charge carriers in electrocatalytic processes. ${ }^{13}$ In particular, the freeze-drying method has been established as the synthetic process for NiFe-LDH/3D graphene since it generates homogeneous porosity, which causes the elevated mechanical stability of the interconnected graphene network. ${ }^{76} \mathrm{NiFe}-\mathrm{LDH} / 3 \mathrm{D}$ graphene has been widely used in various electrocatalytic, ${ }^{76-78}$ photoelectrochemical, ${ }^{60,79}$ and adsorption processes, ${ }^{80}$ but there have been no reports on photocatalytic water splitting activity.

Alternatively, the evolution of the structure and chemical composition can reveal the development of NiFe-LDH/graphene for superior water splitting. In terms of composition, $\mathrm{NiFe-LDH/graphene} \mathrm{can} \mathrm{best} \mathrm{be} \mathrm{compared} \mathrm{with} \mathrm{its} \mathrm{previous}$ types of derivative materials such as metal oxides, ${ }^{81}$ hydroxides $^{82}$ oxyhydroxides, ${ }^{55}$ and bimetallic alloys, etc. ${ }^{55}$ These LDH-based derivative materials inherit the advantages and inherent properties of a 2D layered structure that exhibits competitive electrocatalytic properties. The structural evolution of $\mathrm{NiFe}-\mathrm{LDH} /$ graphene can also start from the bulk NiFe-LDH, ${ }^{27}$ to multifaceted nanostructured morphology including nanosheets (NS), ${ }^{83}$ hollow microspheres, ${ }^{84}$ and hollow nanoprisms, ${ }^{85}$ etc., with more exposed active sites, which appear to control the agglomeration, and enhance the specific surface area. Alternatively, the hierarchical structure of NiFe-LDH including nanoarrays (NAs), ${ }^{86}$ core-shell arrays, ${ }^{87}$ and pyramidal NAs, ${ }^{88}$ are ideal models with enormous mass and electron transportation properties towards water splitting. Therefore, the structural and compositional transition from the bulk $\mathrm{NiFe}-\mathrm{LDH}$ to the NiFe-LDH/graphene nanocomposite could demonstrate superior water splitting activities due to the exposure of new active sites and synergistic interactions among components. Fig. 1 provides insight into the latest update in the development of NiFe-LDH/graphene-based materials for the OER and overall water splitting.

Among the vast investigations in these diverse fields of water splitting, ${ }^{89-91} \mathrm{NiFe}-\mathrm{LDH} / 2 \mathrm{D}$-graphene and 3D-graphene composites have shown their potential towards activities in the electrocatalytic OER, as an anode material in PEC, and in a few cases as a photocatalyst for $\mathrm{H}_{2}$ and $\mathrm{O}_{2}$ evolution. ${ }^{22,33,49-56,59}$ In general, the $\mathrm{NiFe}-\mathrm{LDH} /$ graphene combination gives deep insight into the structural impact of NiFe-LDHs, with the interfacial function of graphene by establishing a relationship between structure and activity. In recent years, there have been reports work based on either NiFe-LDH or NiFe-LDH/graphene for its oxygen electrochemistry; ${ }^{83,92,93}$ however, much emphasis was placed on the distortion of the lattice structure, synthetic methods, morphology and catalytic studies. Fig. 2 shows the systematic trend in the advancements in NiFe-LDH/graphene-based materials, signifying the most recent synthetic methodologies based on different growth approaches and applications in EC, PEC and PC.

\section{Preparation of NiFe-LDH/graphene nanocatalysts}

This section covers the preparation of NiFe-LDH/graphene, based on different growth approaches for superior performance. The growth strategy of NiFe-LDH/graphene is broadly divided into two different approaches: (a) the in situ growth of 
(a)

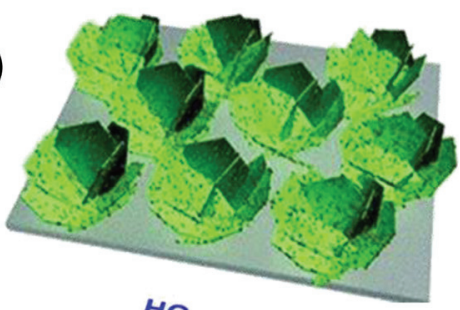

(d)

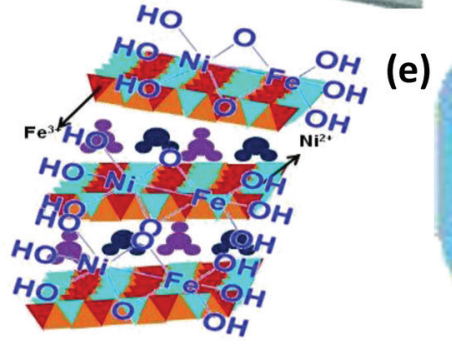

(b)
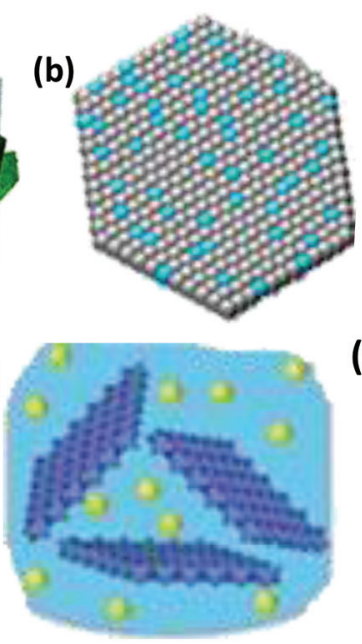

(c)

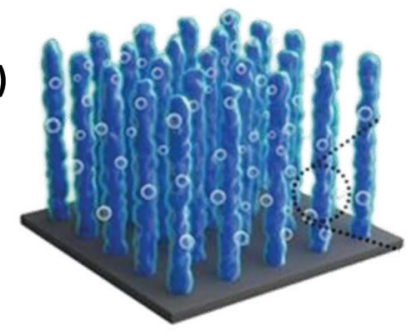

(f)

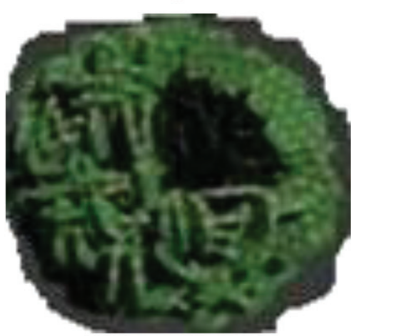

(g)
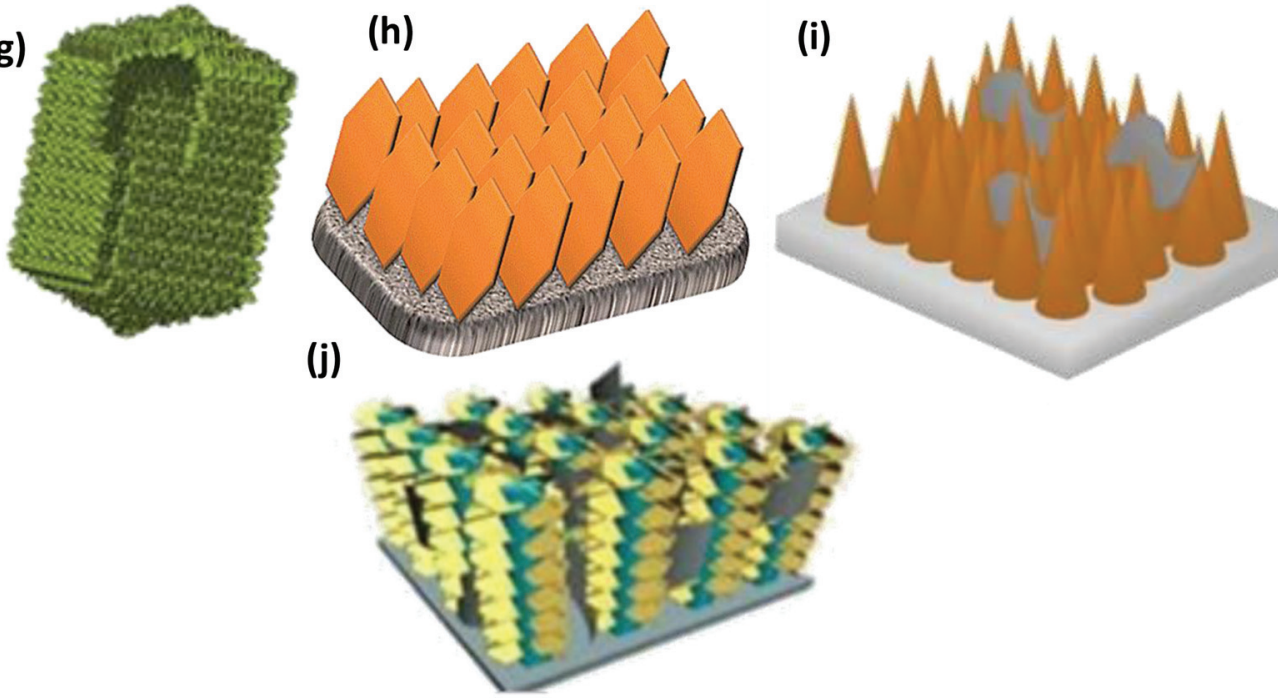

Fig. 1 The structural development of the NiFe-LDH/graphene nanocomposite from the following: (a) metal oxide (e.g., Fe-V@NiO/NF), adapted from ref. 81 with permission from The Royal Society of Chemistry. (b) Hydroxides (e.g. Ni/Ni(OH) $)_{2}$, adapted with permission from ref. 82 , Copyright American Chemical Society. (c) NiFe-oxyhydroxide@NiFe alloy nanowires, adapted from ref. 55 with permission from The Royal Society of Chemistry. (d) Bulk NiFe-LDHs, adapted from ref. 27 with permission from The Royal Society of Chemistry. (e) NiFe-LDH NS, adapted with permission from ref. 83, Copyright American Chemical Society. Nanostructured materials including (f) NiFe-LDH microspheres, adapted with permission from ref. 84, Copyright American Chemical Society. (g) Hollow nanoprisms, reproduced and modified with permission from ref. 85. Copyright 2018, Wiley VCH. (g) NiFe-LDH NS arrays, adapted from ref. 86 with permission from The Royal Society of Chemistry. (h) TiO $2 / \mathrm{rGO} / \mathrm{NiFe}-\mathrm{LDH}$ core-shell NAs, adapted from ref. 87 with permission from The Royal Society of Chemistry. (i) $\mathrm{BiVO}_{4} / \mathrm{rGO} / \mathrm{NiFe}$ pyramidal NAs, adapted from ref. 88 with permission from The Royal Society of Chemistry.

NiFe LDH on graphene support, and (b) the heterostructure assembly of NiFe LDH/graphene.

\section{(a) The in situ growth of NiFe-LDH on graphene support}

The lamellar surface of GO contains functional groups like $-\mathrm{COOH}$ and $-\mathrm{OH}$, which offer an appropriate platform for the in situ growth of NiFe-LDH. ${ }^{94-96}$ The $\mathrm{Ni}^{2+}$ and $\mathrm{Fe}^{3+}$ metal cations as the metal precursors of NiFe-LDH possess a strong affinity for, and bond to, the functional groups on the GO surface. Consequently, adsorption and adhesion of metal cations, then nucleation followed by the growth of NiFe-LDH proceeded by the addition of appropriate precipitating agents.
In situ growth can strengthen the linkage between NiFe-LDH and graphene, forming different morphologies. ${ }^{97}$ In the majority of cases, NiFe-LDH/GO originates from a one-step chemical deposition (solvothermal/hydrothermal method). ${ }^{33,34,64,98}$ It is notable that the morphological and structural aspects of NiFe-LDHs fully depend upon the $\mathrm{OH}^{-}$ concentration through the nucleation step, and nanometric sizes with tuned morphologies of NiFe-LDH/GO can be obtained by controlling the reaction time and temperature. ${ }^{98}$ Lee $e t a l$. were the first to report the one-pot growth approach by growing NiFe-LDH on rGO (NiFe/rGO) by a step-wise solvothermal reaction at $120{ }^{\circ} \mathrm{C}$ for $18 \mathrm{~h}$, and $160^{\circ} \mathrm{C}$ for $2 \mathrm{~h}$ 


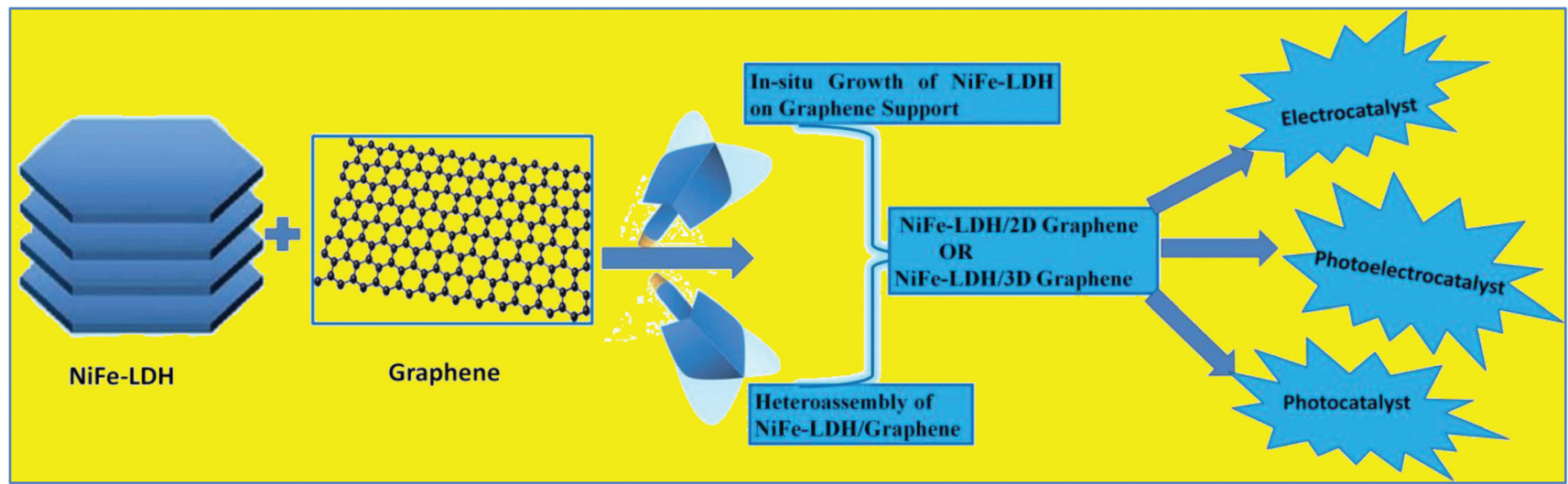

Fig. 2 Scheme of the hierarchical NiFe-LDH/graphene hybrid catalyst with strategies for the synthetic methodology based on two different growth approaches and applications in EC, PEC and PC.

(Fig. 3(a)). ${ }^{99}$ NiFe-LDH was easily crystallized and GO was simultaneously reduced to rGO during the solvothermal reactions. In this approach, NiFe-LDH crystal plates were selectively deposited onto the oxygen functional groups of the rGO layer with higher conductivity and larger surface area. These GOintegrated NiFe-LDH exhibited superior performances in the electrochemical OER and significantly increased the activity of a $\mathrm{Fe}_{2} \mathrm{O}_{3}$ photoanode in PEC activity with high stability. Later on, Kang et al. explored a related work with the $\mathrm{Ni} / \mathrm{Fe}$ cation molar ratio of $5: 1$ and incorporated in GO by using the solvothermal technique (Fig. 3(b)). ${ }^{100}$ However, Kang's group reported more effective OER activity than that reported by Lee

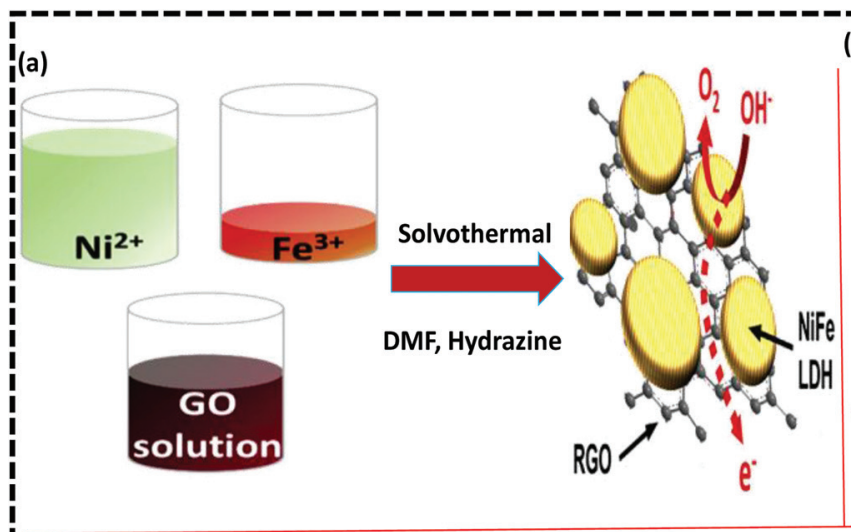

(b)
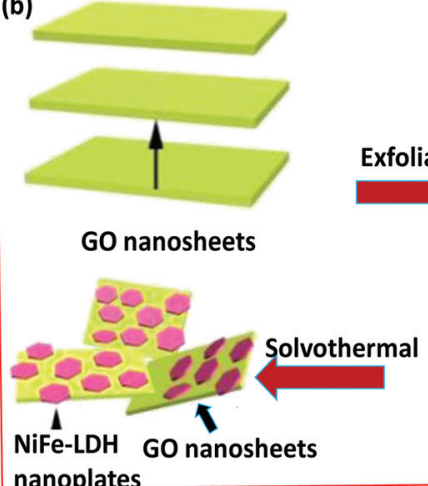
nanoplates
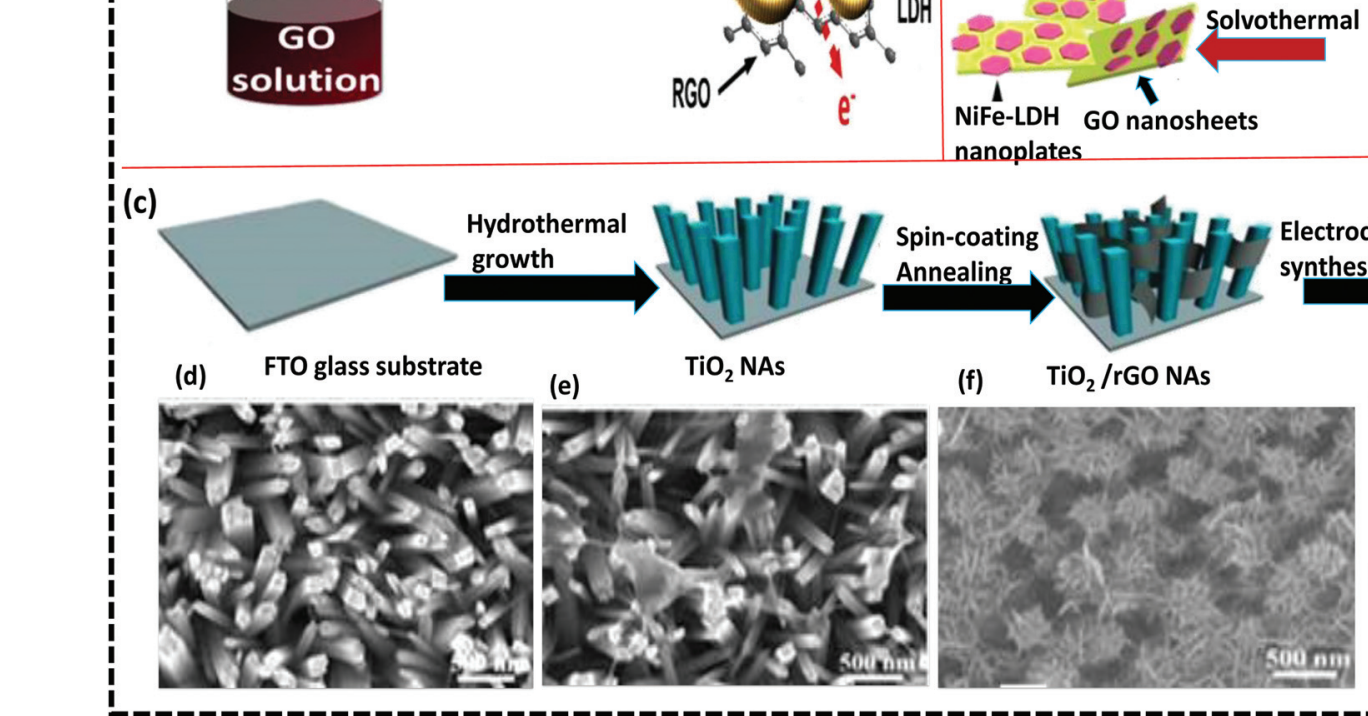

$\mathrm{TiO}_{2} / \mathrm{rGO} / \mathrm{NiFe}-\mathrm{LDH}$ NAs

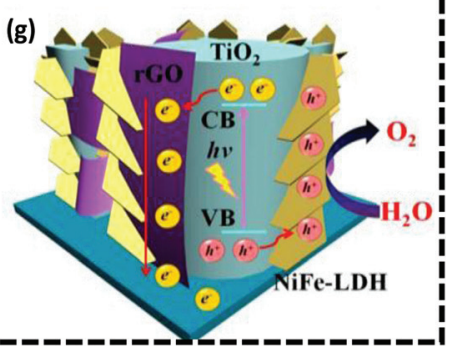

Fig. 3 (a) Illustration of the in situ growth of NiFe LDH on the rGO support for the OER. Reproduced with permission. ${ }^{99}$ Copyright 2015 , Elsevier. (b) The procedure for the formation of $\mathrm{rGO} / \mathrm{NiFe}-\mathrm{LDH}$. Reproduced with permission. ${ }^{100}$ Copyright 2016, Elsevier. (c) The design process of the TiO ${ }_{2} /$ $\mathrm{rGO} / \mathrm{NiFe}-\mathrm{LDH}$ core-shell NAs. (d-f) SEM images of $\mathrm{TiO}_{2}, \mathrm{TiO}_{2} / \mathrm{rGO}$ and $\mathrm{TiO}_{2} / \mathrm{rGO} / \mathrm{NiFe}-\mathrm{LDH}$ NAs. (g) The mechanism of PEC water oxidation in $\mathrm{TiO}_{2} / \mathrm{rGO} / \mathrm{NiFe}-\mathrm{LDH}$ core-shell NAs. ${ }^{87}$ Adapted with permission from the Royal Society of Chemistry. 
et al. The electrophoretic method can also be applied to the in situ growth of NiFe-LDH over the GO surface. Generally, a conductive substrate is used for cathodic electrodeposition using $\mathrm{Ni}^{2+}$ and $\mathrm{Fe}^{3+}$ salts as electrolytes at a constant potential. ${ }^{101}$ The substrate has a strong impact on the nucleation process. The electrophoretic method is an easy, time-saving, and green method, with a prominent path to building 3D LDH NAs. Interestingly, Shao et al. prepared a NA assembly of a photoanode by spin-coating GO NS over the surface of $\mathrm{TiO}_{2}$ NAs; the successive electrodeposition of NiFe-LDH nanoplatelets gave rise to the hierarchical core-shell structure of $\mathrm{TiO}_{2} /$ rGO/NiFe-LDH as shown in Fig. 3(c). ${ }^{87}$ This multistep synthetic approach is reasonably excellent in controlling the composition and morphology by homogeneously anchoring rGO and NiFe-LDH nanoplatelets over the $\mathrm{TiO}_{2}$ NA surface. The scanning electron microscopy (SEM) image reveals a complete fitting of NiFe-LDH nanoplatelets on the total NAs assembly (Fig. 3(d-f)). These ternary $\mathrm{TiO}_{2} / \mathrm{rGO} / \mathrm{NiFe}-\mathrm{LDH}$ NAs exhibited excellent PEC water splitting activities with a maximum current density of $1.74 \mathrm{~mA} \mathrm{~cm}^{-2}$ at $0.6 \mathrm{~V}$ and an efficiency of $0.58 \%$, at $0.13 \mathrm{~V}$. The enhanced PEC activities might be attributed to the synergistic result of strong chemical binding interactions among $\mathrm{TiO}_{2}$, rGO, and NiFe-LDH. Fig. 3(g) displays the mechanism of PEC water oxidation using ternary NAs, which reveals that rGO with a high electron storage tendency, accepts photoinduced electrons of $\mathrm{TiO}_{2}$ and holes are trapped by the NiFe-LDH. Consequently, NiFe-LDH is the active site for water oxidation. Alternatively, the superior electron mobility nature of rGO causes the electrons to rapidly migrate to the PEC current collector. Hence, the role of graphene in this ternary heterostructure is to act like an ideal electron sink, which effectively reduces the bulk recombination and the synergistic effects that enhance the PEC photoconversion efficiency.

The literature study revealed that heteroatom (N, P, S, and B)-doping is an effective approach for increasing the catalytic activities of graphene materials. ${ }^{102}$ Generally, melamine and glucose are used as the nitrogen sources for preparing $\mathrm{N}$-doped graphene. ${ }^{103}$ In our reported work, we have also used melamine as a source for doping $\mathrm{N}$ in graphene and forming g- $\mathrm{C}_{3} \mathrm{~N}_{4}$ in heterostructured $\mathrm{g}-\mathrm{C}_{3} \mathrm{~N}_{4} / \mathrm{N}-\mathrm{rGO} / \mathrm{NiFe}-\mathrm{LDH}{ }^{33}$ These heteroatoms are called "super-hot spots" (SHS) over the surface of graphene. It was proved that these SHS create topology-induced defects, as well as porosity in graphene. It was also revealed that the SHS contributed to the adsorption of metal cations $\left(\mathrm{Ni}^{2+}, \mathrm{Fe}^{3+}\right)$, followed by the in-plane mesoporous graphene matrix acting as a nanoreactor for the spatially confined growth of NiFe-LDH crystals. ${ }^{104,105}$ Benefitting from the advantages of the porous N-doped graphene framework (NGF), Zhang et al. fabricated NiFe-LDH/NGF in a spatial confinement strategy via a one-step urea-assisted precipitation by using $\mathrm{NH}_{3}$ as the source of $\mathrm{N}$, and $\mathrm{MgO}$ as the template for the deposition of NGF (Fig. 4(a)). ${ }^{106}$ The conductive NGF substrate consisting of a mesoporous framework acts as a medium for the in situ growth of ultra nanostructured NiFe-LDH (nNiFe-LDH). It was ascertained that the $\mathrm{N}$-doped graphene induces topological defects that contribute to the adhesion of $\mathrm{Ni}^{2+}$ and $\mathrm{Fe}^{3+}$ metal cations and promote nucleation and thereby, the mesoporous

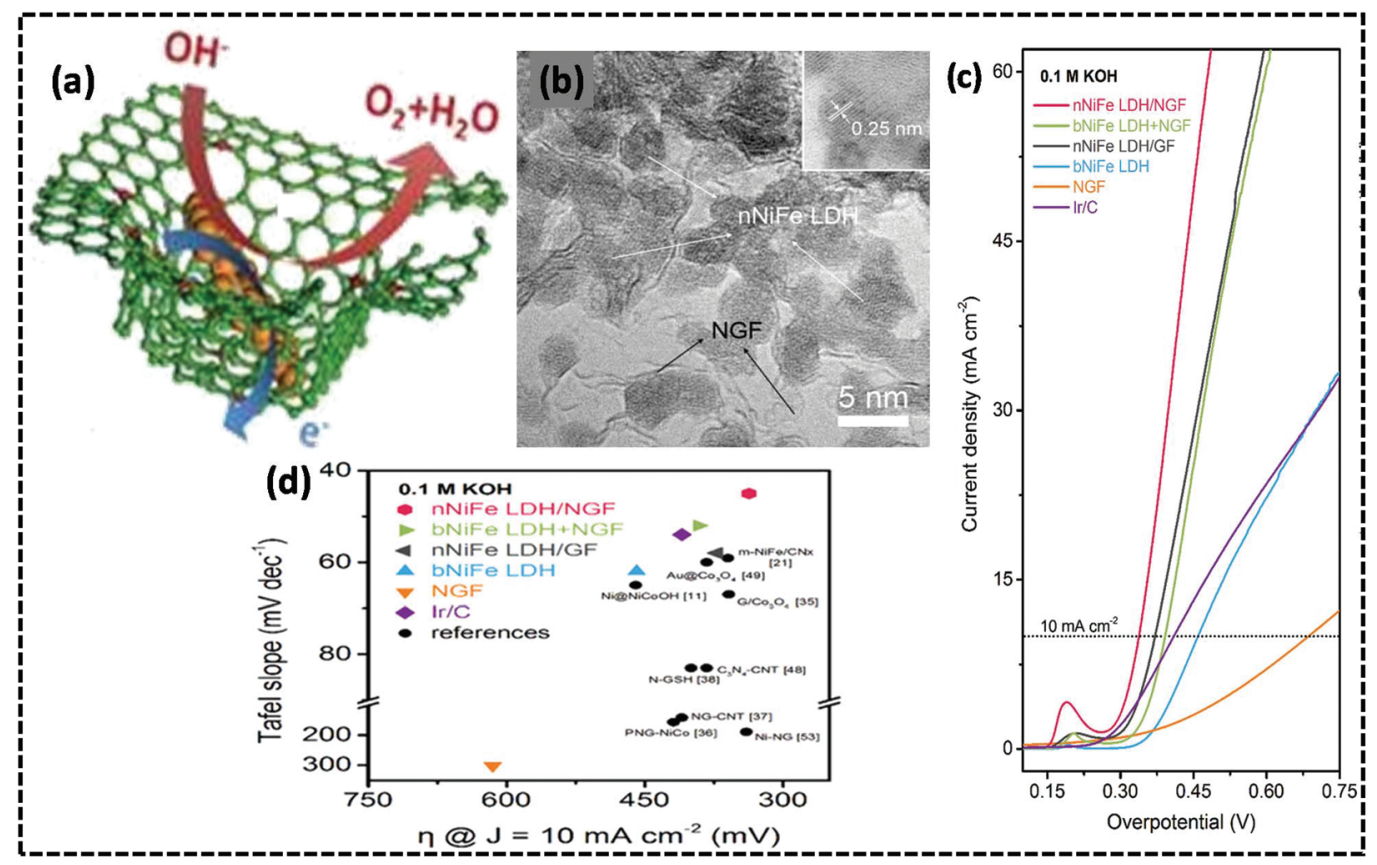

Fig. 4 (a) Diagrammatic representation of the spatially confined hybrid structure of nNiFe-LDH/NGF. (b) HRTEM image of nNiFe LDH/NGF hybrids displaying the homogeneous deposition of nNiFe-LDHs in the NGF matrix. (c) LSV curves of the nNiFe-LDH/NGF series and related materials in $0.1 \mathrm{M}$ $\mathrm{KOH}$ electrolyte. (d) The subsequent Tafel plots. Reproduced and modified with permission from ref. 106. Copyright 2015, Wiley VCH. 
in-plane graphene acts as a nanoreactor to perform the spatially confined growth and homogeneous dispersion of nNiFe-LDH on the support of the mesoporous graphene framework. ${ }^{102}$ The confined growth of nNiFe-LDH within the mesopores of NGF leads to NS of size less than $10 \mathrm{~nm}$ (Fig. 4(b)). This process signifies the controlled hybridization of the active phases of nanometric NiFe-LDH into the conductive NGF substrate to form the $\mathrm{nNiFe}-\mathrm{LDH} / \mathrm{NGF}$ hybrid with strong interfacial interactions, which fully exposes the electrochemically active surface area for to facilitate the OER activities (Fig. 4(c)). It is the NGF that acts as a conducting substrate with a high potential for superior charge transport in electrocatalytic reactions. The X-ray photoelectron spectroscopy (XPS) data of $\mathrm{nNiFe}-\mathrm{LDH} / \mathrm{NGF}$ reveals a higher binding energy shift of Ni $2 \mathrm{p}$ (ca. $0.7 \mathrm{eV}$ ), signifying the linkage of the $\mathrm{C}-\mathrm{N} \cdots \mathrm{Ni}-\mathrm{O}$ bond and strong local electronic coupling between NiFe-LDHs and graphene will occur. Consequently, the intrinsic catalytic activities of NiFe-LDHs are tuned with the exposure of active phases like $\gamma-\mathrm{Ni}_{1-x} \mathrm{Fe}_{x} \mathrm{OOH}$ or $\gamma$-NiOOH, which promotes intermediate adsorption for the OER performance. ${ }^{55,56}$ Accordingly, the hierarchical structure of the NiFe-LDH/NGF displayed a more kinetically active OER catalyst than Ir/C and other reported materials, with extensive durability in alkaline medium. Important OER parameters include a lower Tafel slope (45 mV $\mathrm{dec}^{-1}$, Fig. 4(d)), small overpotential (337 mV) at boosting the current density of $10 \mathrm{~mA} \mathrm{~cm} \mathrm{~cm}^{-2}$ in $0.10 \mathrm{M} \mathrm{KOH}$. Overall, the better OER activity of nNiFe-LDH/NGF could be due to the synergistic effect of LDH and NGF with unique structural features due to the involvement of the conductive mesoporous NGF, which facilitates electron and mass transfer through multiple electronic paths during the OER. Most importantly, the spatially confined growth of nanosized NiFe-LDHs forming a strong hybrid with graphene and generating open coordinate sites and lengthening the edges with exposed surface active sites is responsible for the superior OER performance.

In addition to the above advantages including the high conductivity of the $\mathrm{N}$-doped graphene, another approach is the design of various hierarchical porous structures (such as sphere mesopores, wrinkled mesopores, and strutted macropores) by using a surfactant to improve the potential in the OER. ${ }^{107}$ By utilizing the benefit of the porosity of a material for superior catalytic activities, Zhan et al. reported mesoporous $\mathrm{NiFe}-\mathrm{LDH} / \mathrm{NrGO}$ nanospheres by using protonated $\mathrm{g}-\mathrm{C}_{3} \mathrm{~N}_{4} \mathrm{NS}$ ( $\mathrm{p}$-CNNS) as a rich source for $\mathrm{N}$-doping and sodium dodecyl sulfonate (SDS) as a structure-directing agent in a one-pot solvothermal template method (Fig. 5(a)). ${ }^{72}$ The creation of the hierarchical mesoporous/nanospheres could be mainly credited to the reverse micelle effect. The overall catalytic activities of NiFe-LDH/N-rGO in the ORR and OER confirmed its tolerance for long-term durability. The creation of $3 \mathrm{D}$ porous nanospheres in NiFe-LDH/N-rGO bestowed it with superior activity due to the increase in the number of active sites and faster mass transfer. Graphene aerogels (GA) also have the advantages of multidimensional electronic channeling and tangled hierarchical porous block structure (mesoporous, and microporous) with high specific surface area, appropriate for the growth of NiFe-LDH. ${ }^{107}$ GA also possesses high electrical conductivity and better mechanical properties, which facilitate rapid mass transport of opposite charges, and easy catalyst
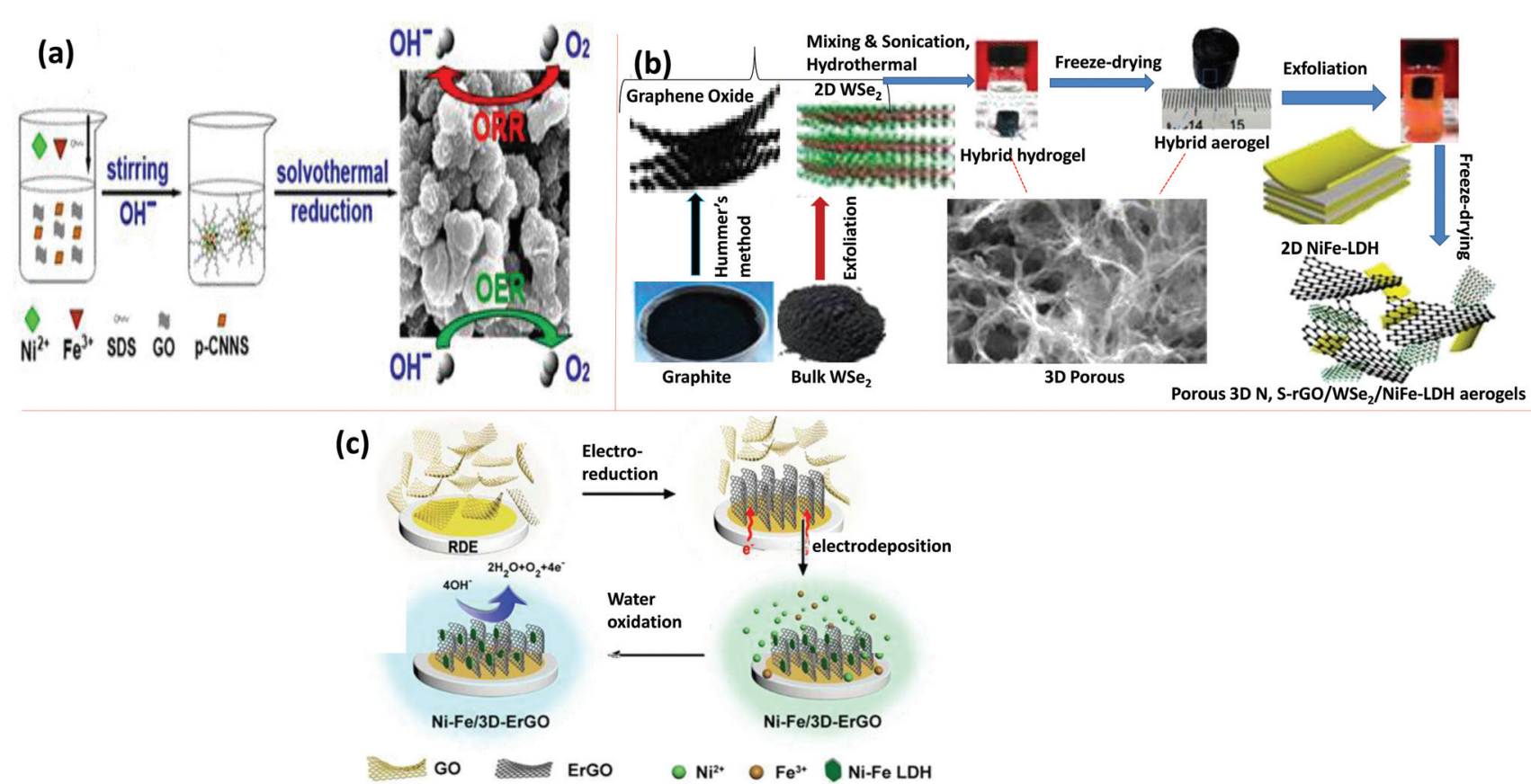

Fig. 5 (a) Schematic diagram for the synthesis of the NiFe-LDH/NrGO catalyst. Reproduced with permission. ${ }^{72}$ Copyright 2017, Elsevier. (b) Synthetic steps of N,S-rGO/WSe $2 / \mathrm{NiFe}-\mathrm{LDH}$. Adapted with permission from ref. 79 a Copyright 2017, American Chemical Society. (c) The design process and application of the NiFe/3D-ErGO electrode. ${ }^{78}$ Adapted with permission from the Royal Society of Chemistry. 
recyclability is another merit. $\mathrm{Xu}$ et al. prepared porous $3 \mathrm{D} \mathrm{N}$, $\mathrm{S}-\mathrm{rGO} / \mathrm{WSe}_{2} / \mathrm{NiFe}-\mathrm{LDH}$ aerogels by assembling NiFe-LDH NS with GA via a simple hydrothermal and electrostatic selfassembly technique as shown in Fig. 5(b). ${ }^{79}$ Along these lines, scientists are also capable of designing the one-step synthesis of NiFe-LDH/GA. Despite the exciting experiments in the design of various catalysts for use in electrocatalytic and PEC applications, the higher overpotential, durability and specific current density are the key factors for determining the catalyst potential at specific reactions. The in situ deposition of material over a 3D conductive substrate to design a catalyst or directly depositing the catalyst over a film or a conductive substrate is expected to be more reactive than directly dripping materials on GC electrodes during the activity test. In nanocomposites, the buffering of the electrolyte in a 3D hierarchically macroporous structure increases the ion mobility and utilizes the active species more frequently to facilitate the diffusion of gas bubbles. The most efficient 3D conductive substrates for growing materials are Ni foam, ${ }^{107}$ graphene hydrogels, ${ }^{108} \mathrm{CFP},{ }^{109}$ and the Au-rotating disk electrode (Au-RDE), ${ }^{78}$ or some other metal foil/mesh with carbonaceous substrate were also taken in the precursor metal salt solution, so that the corresponding NiFe-LDH could be developed on it. Shi et al. designed a novel electrode by using NiFe-LDH and 3D electrochemically active rGO (NiFe-LDH/ 3DErGO), supported on Au-RDE by electrochemical reduction of dispersed GO sheets in aqueous electrolyte, and next electro- chemical deposition of NiFe-LDH nanoplates on the 3D-ErGO substrate (Fig. 5(c)). ${ }^{78}$

Besides the effects of the heteroatom-doped porous graphene, graphene aerogel and free-standing electrodes, the coupling of NiFe-LDH with GO hydrogel is another novel strategy for boosting the electrocatalytic OER performances. Gu et al. used the GO hydrogel matrix supported over Ni foam by the doctor blade method, which was subsequently freezedried, to produce the 3D-porous GO matrix (Fig. 6). ${ }^{75} \mathrm{NiFe}$ $\mathrm{LDH}$ nanoflakes were attached to the 3D-GO matrix followed by the in situ reduction in a hydrothermal reaction to form 3Dporous rGO/NiFe-LDH supported on $\mathrm{Ni}$ foam (NiFe-LDHs/ NF@3D-rGO). The material prepared in this method had a high degree of porosity along with good mechanical strength. The designed 3D-rGO/NiFe-LDH demonstrated excellent OER performance under alkaline medium at the current density of $20 \mathrm{~mA} \mathrm{~cm}^{-2}$, with a small Tafel slope of $57 \mathrm{mV} \mathrm{dec}^{-1}$, and overpotential of $170 \mathrm{mV}$.

\section{(b) Heteroassembly of NiFe-LDH/graphene}

The heterostructure is the interface formed due to band alignment among two or more semiconductors with unequal band structure, which possesses intrinsic properties of the countercomponents with considerably enhanced performances. In particular, the heterostructure coupling of NiFe-LDH/graphene synergistically played a vital role in manipulating the band

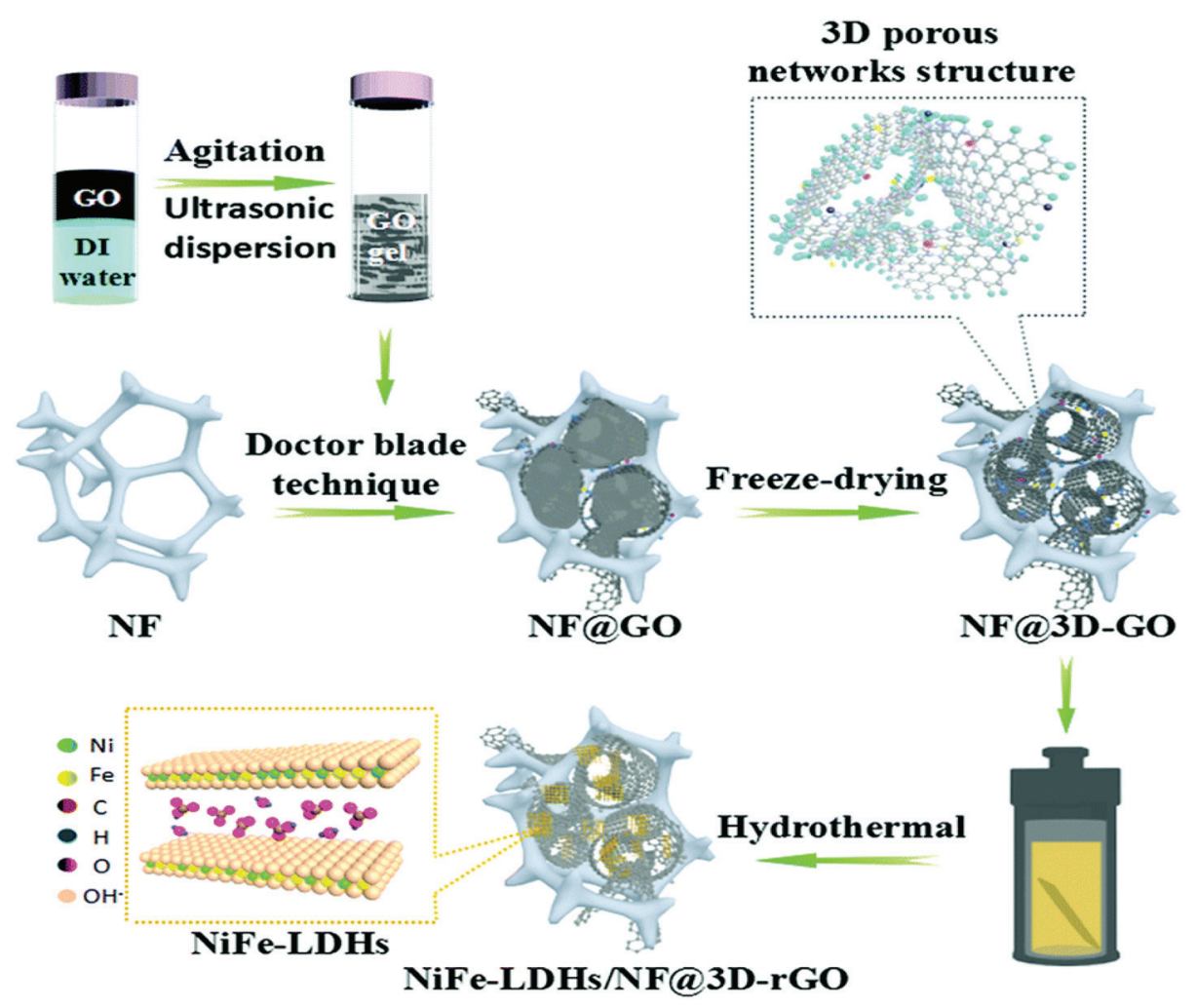

Fig. 6 Illustration of the preparatory steps of NiFe-LDHs nanoflakes anchored on a 3D porous rGO framework supported on Ni foam (NiFe-LDHs/ NF(a3D-rGO). Reproduced from ref. 75 with permission from the Centre National de la Recherche Scientifique (CNRS) and the Royal Society of Chemistry. 
alignment and electronic band structure and facilitated the excessive electron transfer through an interconnected electron highway that promoted water splitting reactions. ${ }^{110}$ In recent years, exfoliation has been recognized to be a simple method for generating thin layer NS with a theoretically calculated higher specific surface area of $1000 \mathrm{~m}^{2} \mathrm{~g}^{-1}$, which is associated with huge quantities of exposed active sites for enhanced catalytic efficiency. Solid NiFe-LDH, possesses strong interplane chemical bonds but weaker out-of-plane van der Waals forces. ${ }^{111} \mathrm{NiFe}-\mathrm{LDH}$ can be exfoliated and self-assembled with other layered materials like graphene, such as $\mathrm{g}-\mathrm{C}_{3} \mathrm{~N}_{4}$ and $\mathrm{MoS}_{2}$, to form heterostructured materials. We have also reported different heterostructured materials of NiFe-LDH by the exfoliation strategy, composited with other layered materials such as in $\mathrm{g}-\mathrm{C}_{3} \mathrm{~N}_{4} / \mathrm{NiFe}-\mathrm{LDH},{ }^{27} \mathrm{Ag} @ \mathrm{Ag}_{3} \mathrm{PO}_{4} / \mathrm{g}-\mathrm{C}_{3} \mathrm{~N}_{4} /$

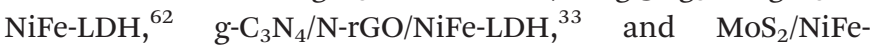
LDH. $^{34}$ The simplistic exfoliation strategy of NiFe-LDHs can alter the electronic band structure and generate more active sites with higher conductivity, thus radically increasing the potential catalytic activity and stability. In the past years, various methods for exfoliating a material have been cited in the literature, such as liquid-phase, plasma-induced, and solid-phase exfoliation, etc. ${ }^{7}$ Our recent work also demonstrated the caliber of the exfoliation technique in the design of the ternary $\mathrm{NiFe}-\mathrm{LDH} / \mathrm{N}-\mathrm{rGO} / \mathrm{g}-\mathrm{C}_{3} \mathrm{~N}_{4}$ heterostructure. This ternary heterostructure was synthesized by multistep calcination-electrostatic self-assembly and hydrothermal routes. ${ }^{33}$ The HRTEM image manifests the intercalation of $\mathrm{N}$-rGO at the interface of the NiFe-LDH/g- $\mathrm{C}_{3} \mathrm{~N}_{4}$ nanohybrid (Fig. 7). ${ }^{33}$ The extraordinary photocatalytic activity of this ternary hetero- structure towards $\mathrm{PC} \mathrm{H}_{2}$ and $\mathrm{O}_{2}$ evolution along with effectiveness towards the degradation of RhB dye and phenol might be due to the synergistic electrostatic coupling interactions of the constituent semiconductors and the unique role of N-rGO as a mediator or electron transport bridge in the effective charge separation through the $Z$-scheme mechanistic pathway.

As stated above, the electrostatic self-assembly method is a viable technique generally used for the construction of the $\mathrm{NiFe-LDH/graphene} \mathrm{heterostructure.} \mathrm{It} \mathrm{utilizes} \mathrm{the} \mathrm{opposite}$ surface charge of two positively and negatively charged materials bonded through electrostatic forces of interaction. The aqueous suspension of graphene is negatively charged, while the positively charged host layer of NiFe-LDHs is built up with an orderly arrangement of metal cations and these are the fundamental properties for designing the heteroassembly of NiFe-LDH/graphene via electrostatic self-assembly. ${ }^{33}$ In 2014, Yang and Long et al. reported the strongly coupled NiFe$\mathrm{LDH} / \mathrm{GO}$ heterostructure by the anion $\left(\mathrm{CO}_{3}{ }^{2-}\right.$ or $\left.\mathrm{Cl}^{-}\right)$intercalation method to enlarge the interlayer space of NiFe-LDH, followed by exfoliation and self-assembly with GO sheets. ${ }^{112}$ The opposite stacking of NiFe-LDH and GO NS resulted in enormous exposure of the large active surface with higher conductivity (Fig. 8(a)). Further progress was acquired by the reduction of the heteroassembled GO to rGO (named FeNi-GO/ rGO LDH) for the high electrocatalytic OER performance. The SEM morphologies of FeNi-Cl LDH and FeNi-GO LDHs revealed rough surfaces and smaller platelets as compared to FeNi-CO $\mathrm{CO}_{3} \mathrm{LDH}$ (Fig. 8(b-d)). The reduction of GO in FeNi-rGO LDH was further confirmed by the XPS spectra and XRD patterns. It is worth mentioning that the as-synthesized FeNi-GO

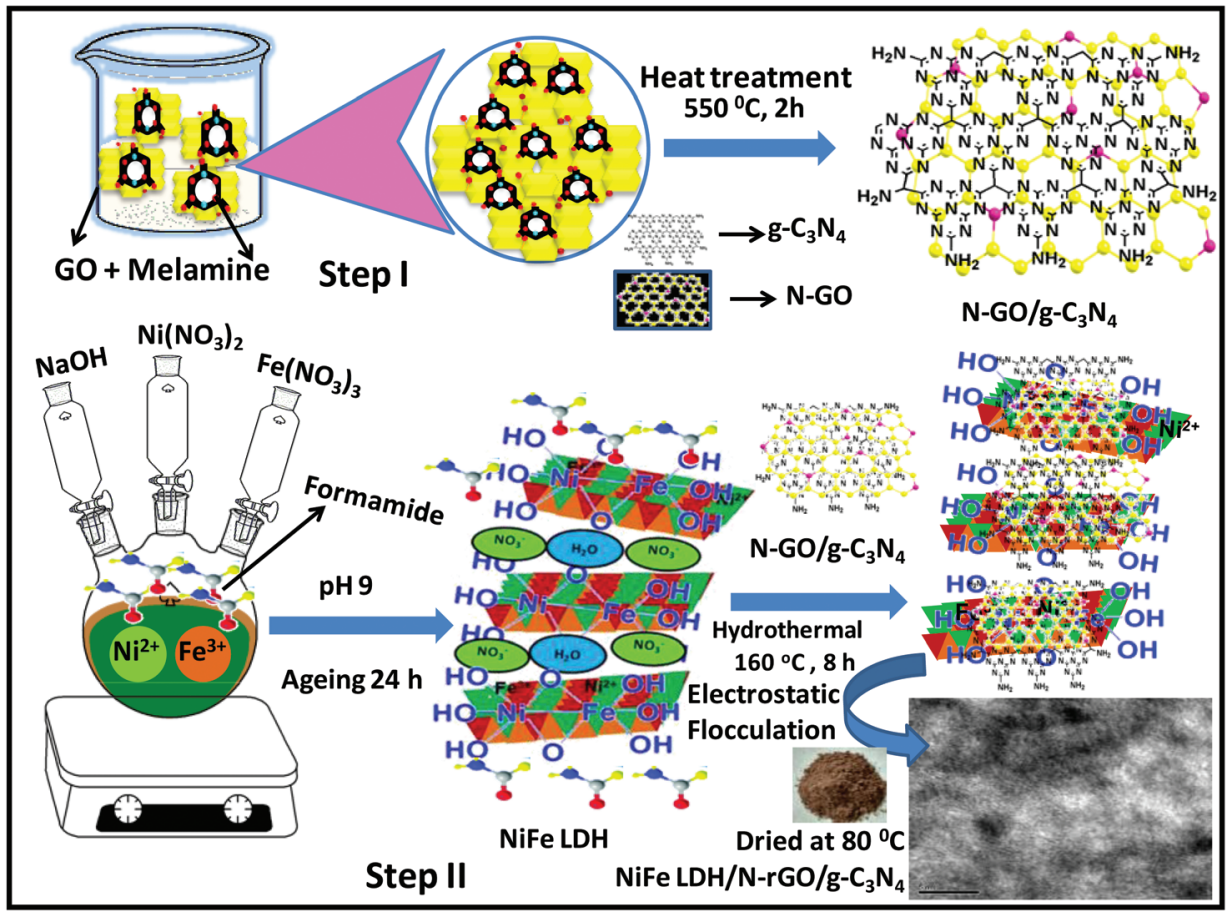

Fig. 7 Synthetic methods of the NiFe-LDH/N-rGO/g- $\mathrm{C}_{3} \mathrm{~N}_{4}$ nanocomposite. Adapted with permission from ref. 33, Copyright 2019, Nature. 

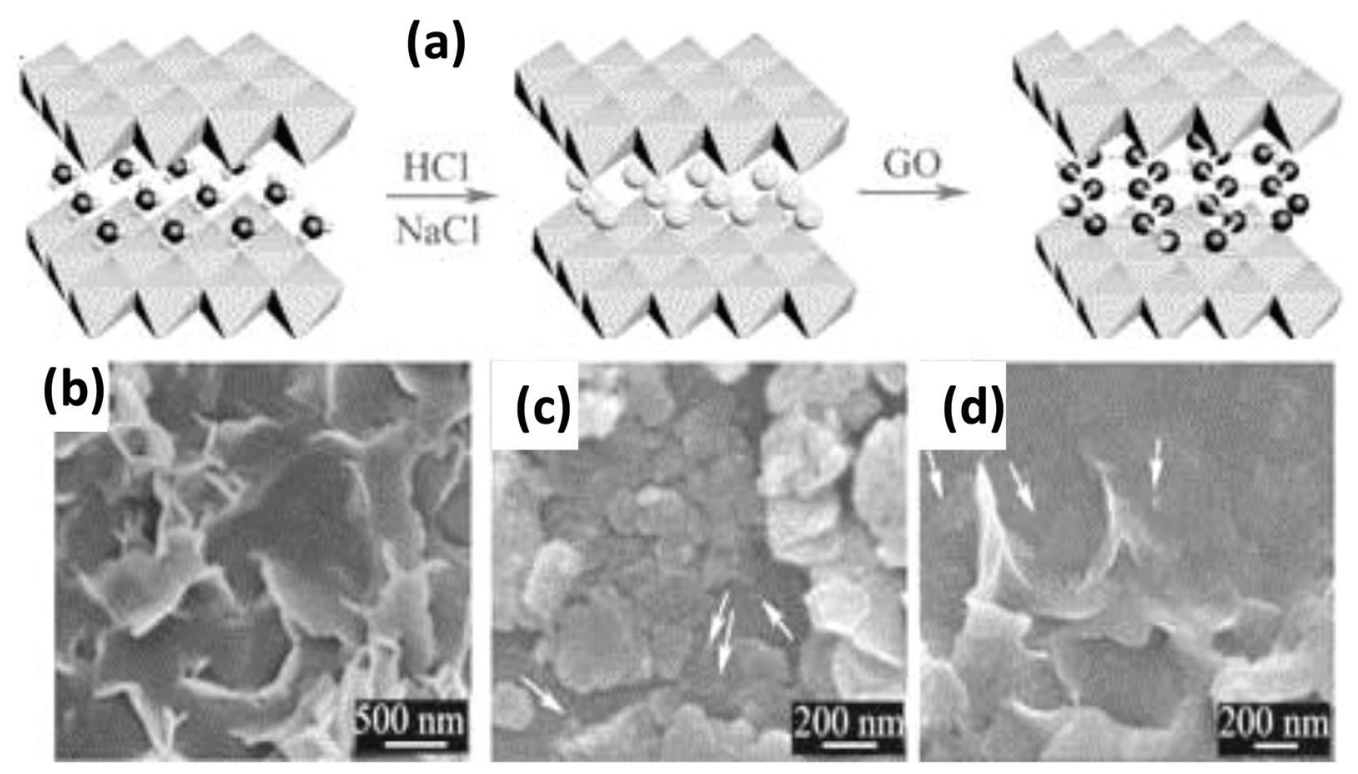

Fig. 8 (a) Fabrication process of the NiFe-LDH/GO hybrids. (b-d) SEM images of $\mathrm{NiFe}-\mathrm{CO}_{3} \mathrm{LDHs}, \mathrm{NiFe}-\mathrm{Cl} \mathrm{LDHs}$, and NiFe-LDH/GO hybrids. Reproduced and modified with permission from ref. 112. Copyright 2014, Wiley VCH.

LDH showed an incremental basal spacing of $1.1 \mathrm{~nm}$, more than twice that of $\mathrm{FeNi}^{-\mathrm{CO}_{3}} \mathrm{LDH}(0.75 \mathrm{~nm})$, which shows the intercalation of GO into the FeNi-LDH. The extended basal spacings of FeNi-LDH permit the diffusion and association of the electrolyte and reactants. The synergistic strong interaction between the stacked rGO layers hybridized with the interlayer of FeNi-LDH by the layer-by-layer assembly facilitated charge transport through the exposed active sites of FeNi-LDH on the conductive rGO layers and thereby led to the favorable OER performance.

Hybrid structures consisting of catalytically active species and conductive supports are the best strategy for improving the electrocatalytic performance. By this approach, constructing a 2D superlattice structure for achieving targeted activity could be far better than preparing simple composites in which lower intimate contact is possible between the active edges and the conductive surfaces, which lead to less migration of electrons or less ion transfer. Sasaki et al. fabricated the NiFeLDH/GO superlattice by a facile delamination strategy (Fig. 9(a)). ${ }^{83}$ In this approach, positively charged NiFe-LDH NS were produced by exfoliating the bulk counterpart in formamide. In the meantime, negatively charged GO NS were synthesized by exfoliating bulk graphite via a modified Hummers' method. ${ }^{113}$ Afterwards, the superlattice NiFe-LDH/rGO nanostructure was realized by the electrostatic flocculation between the electrocatalytic active sites of NiFe-LDH and the conductive $\mathrm{GO} / \mathrm{rGO}$ surface through electrostatic interaction forces. In this process, a synergistic effect from the layer-by-layer assembly between NiFe-LDH and GO with interfacial hybridization is responsible for the formation of the superlattice structure. Accordingly, the NiFe-LDH/rGO superlattice has the advantages of the redox potential of NiFe-LDH and the conductive nature of graphene, which facilitate the achievement of a small Tafel slope of $40 \mathrm{mV} \mathrm{dec}{ }^{-1}$ at the overpotential of $0.217 \mathrm{~V}$, with a current density of $10 \mathrm{~mA} \mathrm{~cm} \mathrm{~cm}^{-2}$, and allow this material to function as a NiFe-based electrocatalyst. Similarly, the CoAl-LDH/GO superlattice was prepared by the layer-by-layer assembly and was used as a potential electrode material for supercapacitor devices. ${ }^{114}$ In contrast to other superlattice structures, $\mathrm{MoS}_{2}$ /graphene was prepared by utilizing the advantages of both the metallic $1 \mathrm{~T}$ phase $\mathrm{MoS}_{2}$ NS and graphene NS, which showed excellent properties in the HER and sodium storage. ${ }^{115}$

In addition to the aforementioned superlattice heterostructure, the $3 \mathrm{D}$ array-like heterostructure is considered to be an emerging material that fully exposes all active sites, which is most effective for energy conversion. However, the stability of the unilamellar NiFe-LDH NS in the 3D array-like heterostructure is still challenging. The stability and directional growth evolution of monolayer LDH NS on GO (horizontal spreading or vertical alignment) can be well controlled by the solid-state exfoliation strategy. By the use of a solid-phase exfoliating material (SPEM) such as a polyhydroxy material, Shen et al. exfoliated NiFe-LDH and GO by using the solid-phase exfoliation strategy to form the 3D array-like NiFe-LDH/GO heterostructure (Fig. 9(b)). ${ }^{73}$ This process revealed the dynamic growth evolution of NiFe-LDH NS on GO NS (horizontal spreading or vertical alignment or both) and provided information about the fully exposed active sites for interfacial interactions. The NiFe-LDH/RGO heterostructure with the vertical growth of NiFe-LDH NS displayed superior electrocatalytic OER activity with a small Tafel slope of $49 \mathrm{mV} \mathrm{dec}^{-1}$ and overpotential of $273 \mathrm{mV}$ at exchange current density of $30 \mathrm{~mA} \mathrm{~cm}{ }^{-2}$ under basic medium. The superior electrocatalytic OER activity of NiFe-LDH/RGO could be accredited to the strong interfacial coupling interactions between NiFe-LDH and RGO NS, as well as the synergistic effect. All these strategies mentioned in this 


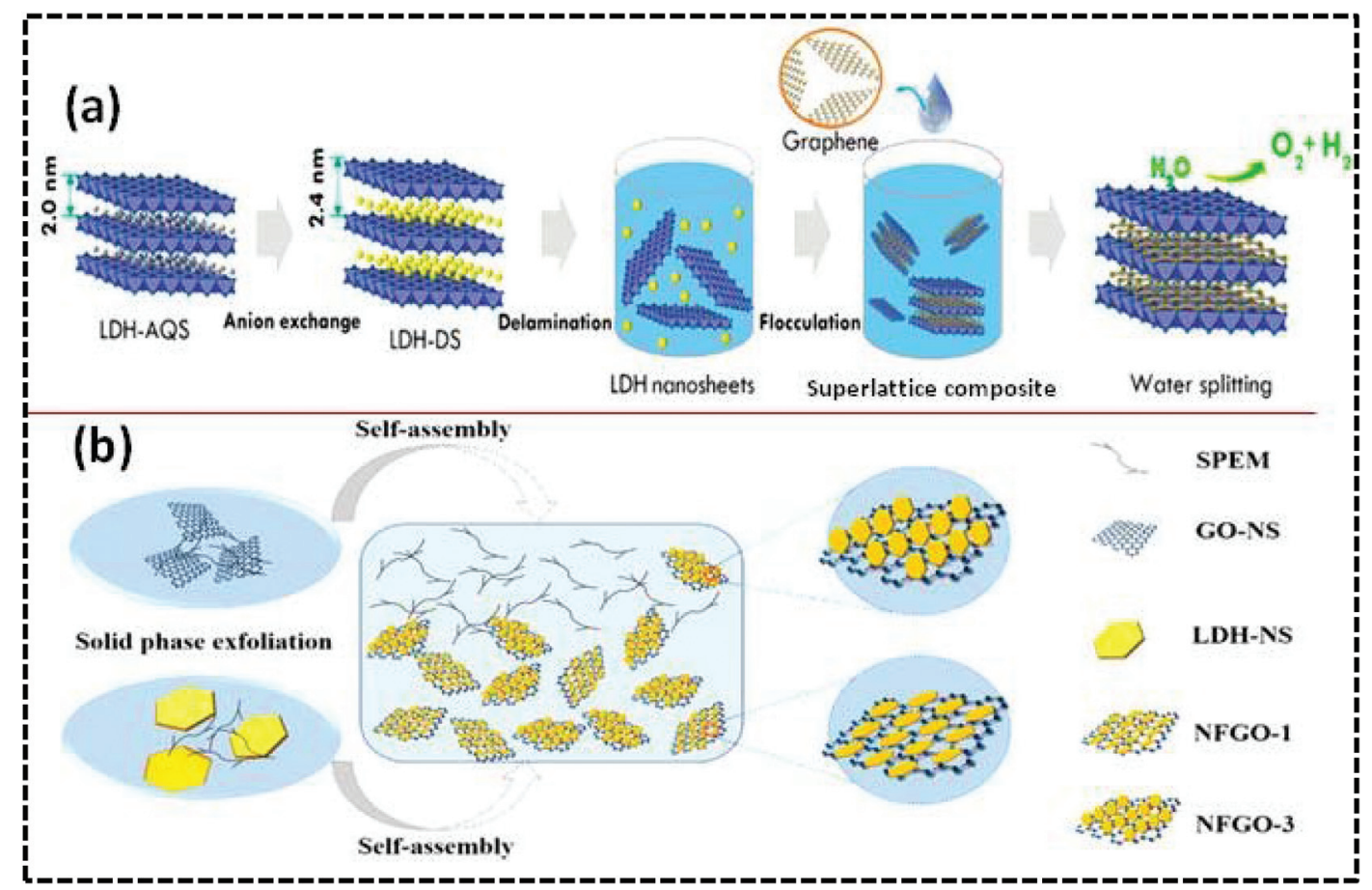

Fig. 9 (a) Illustration of the heterostructure formation of NiFe-LDH NS and graphene for water splitting. Reproduced with permission from ref. 83, Copyright 2015, American Chemical Society. (b) Synthetic steps of NiFe-LDH/GO nanohybrids by using the solid-phase exfoliation-liquid-phase assembly strategy. Reproduced with permission from ref. 73, Copyright 2019, American Chemical Society.

section, including the growth of highly intrinsic LDHs on conductive graphene or the heteroassembly, provide effective methods with morphological tuning, directional growth, contact sites, reaction conditions, and reaction parameters, which are important parameters that determine the formation of NiFe-LDH/graphene hybrid for diverse performances.

\section{Applications of NiFe-LDH/graphene}

The ordered structure and tunable compositions of NiFe-LDHs furnished innovative ideas for the design of extremely competent catalysts for applications in EC, PEC, and PC. ${ }^{27,87,116,117}$ GO is a carbonaceous material responsible for enhancing electron conductivity, as well as providing an enormous surface area for the homogeneous growth of NiFe-LDHs. In this section, we mainly emphasize the applications of NiFe-LDHs/ graphene in the electrocatalytic OER, and the photoelectrochemical and photocatalytic $\mathrm{H}_{2}$ and $\mathrm{O}_{2}$ evolution reactions.

\section{OER catalysis}

Water electrolysis is the most exciting scientific achievement due to the simplistic path for electrochemically breaking of $\mathrm{H}_{2} \mathrm{O}$ into clean $\mathrm{H}_{2}$ and $\mathrm{O}_{2}$. The only starting material is $\mathrm{H}_{2} \mathrm{O}$ and low-cost electricity from solar, wind and hydropower or photovoltaics. ${ }^{4-11}$ Generally, water electrolysis is carried out in a quartz cell consisting of a cathode and anode in which the
HER and OER take place. ${ }^{118}$ The HER is a simple electrochemical reaction that requires a lower overpotential to drive the kinetics of the reduction reactions at the surfaces of many metals. The OER is the most important half-cell reaction for water electrolysis. It involves four proton-coupled electron transfer pathways $\left(4 \mathrm{e}^{-}\right.$transfer and $4 \mathrm{H}^{+}$removal from $\left.\mathrm{H}_{2} \mathrm{O}\right)$, with the sluggish kinetics and high overpotential that decrease the efficiency of the overall water splitting. It is preferred that molecular $\mathrm{O}_{2}$ be generated from the metal oxide surface rather than neat metal, and therefore its mechanism is quite elusive and confined to specific systems. The majority of metals are transformed into hydroxides or oxides throughout an OER process; however, stability in a harsh acidic medium remains challenging, excluding $\mathrm{IrO}_{2}$ and $\mathrm{RuO}_{2}$. LDHs systems are very sensitive to acidic media and hence, the OER of LDHs is normally conducted in alkaline media. Usually, the protons couple in four steps and the electron transfer routes in heterogeneous electrocatalysis in alkaline media occur as follows:

$$
\begin{gathered}
{[\mathrm{M}]+\mathrm{OH}^{-} \leftrightarrow[\mathrm{M}-\mathrm{OH}]+\mathrm{e}^{-}} \\
{[\mathrm{M}-\mathrm{OH}]_{\mathrm{ads}}+\mathrm{OH}^{-} \leftrightarrow[\mathrm{M}-\mathrm{O}]+\mathrm{H}_{2} \mathrm{O}+\mathrm{e}^{-}} \\
{[\mathrm{M}-\mathrm{O}]_{\mathrm{ads}}+\mathrm{OH}^{-} \leftrightarrow[\mathrm{M}-\mathrm{OOH}]+\mathrm{e}^{-}} \\
{[\mathrm{M}-\mathrm{OOH}]_{\mathrm{ads}}+\mathrm{OH}^{-} \leftrightarrow[\mathrm{M}-\mathrm{OO}]+\mathrm{H}_{2} \mathrm{O}} \\
{[\mathrm{M}-\mathrm{OO}]_{\mathrm{ads}}+\mathrm{OH}^{-} \leftrightarrow \mathrm{M}+\mathrm{O}_{2}+\mathrm{e}^{-}}
\end{gathered}
$$

Unlike the HER, a low overpotential of $10 \mathrm{~mA} \mathrm{~cm}{ }^{-2}$, a small Tafel slope, higher reaction dynamics to facilitate the multi- 
step electron transfer, and easily available metal-containing active sites are the main criteria that determine the potential of an electrocatalyst to satisfy the OER. ${ }^{119}$ The Tafel slope is very much dependent on the applied potentials. At a higher positive potential and higher Tafel slope, the electrode becomes positive due to the availability of $\mathrm{OH}^{-}$. Consequently, the rate-determining steps of the OER involve the formation of $\mathrm{M}-\mathrm{O}$ bonds or the adsorption of $\mathrm{OH}^{-}$. Similarly, at a smaller Tafel slope, the rate-determining step involves the formation of $\mathrm{M}-\mathrm{O}$ and $\mathrm{M}-\mathrm{O}-\mathrm{O}$ bonds, respectively. However, the OER suffers from intrinsically sluggish kinetics and larger overpotentials. To date, noble metal catalysts such as $\mathrm{RuO}_{2}$ and $\mathrm{IrO}_{2}$ have been used in the OER, but they are costly and suffer from instability issues. Current research has explored the potential of earth-abundant transition metals and their derived compounds to replace these noble metals for the OER. ${ }^{120}$ Since $\mathrm{Ni}^{2+}$ and $\mathrm{Fe}^{3+}$ are the most suitable transition metal cations for the OER, NiFe alloys have been reported to exhibit the electrocatalytic OER. ${ }^{52-54}$ Alternatively, the NiFe-LDHs catalysts are the most appropriate candidates for the electrocatalytic OER due to their tunable structure, composition and stability in the electrochemical OER. Candelaria et al. explored the impact of $\mathrm{Fe}$ in controlling the electrocatalytic activities of NiFe nanoparticles (NP). ${ }^{121}$ Bimetallic FeNi NP evolved with a core-shelllike structure, consisting of $\mathrm{Fe}(\mathrm{OH})_{3}$ or $\alpha-\mathrm{Fe}_{2} \mathrm{O}_{3}$ as the core and $\mathrm{Ni}(\mathrm{OH})_{2}$ integrated with metallic $\mathrm{Fe}$ as the shell. Monometallic Ni NP contains both $\beta-\mathrm{Ni}(\mathrm{OH})_{2}$ and $\alpha-\mathrm{Ni}(\mathrm{OH})_{2}$ with a consider- able quantity of metallic Ni NP. Interestingly, bimetallic FeNi NP was found to have a significantly higher OER activity than the monometallic $\mathrm{Ni}$ and Fe due to the Fe doping in $\mathrm{Ni}(\mathrm{OH})_{2} /$ $\mathrm{NiOOH}$. Zhang et al. subsequently reported a highly competent NiFe film that exhibited excellent OER activities; ${ }^{122}$ specifically, $\mathrm{Fe}^{3+}$ was the main modulator for controlling the valence states of $\mathrm{Ni}$ in the electrocatalytic processes. The surface defects created by the doping of $\mathrm{Fe}^{3+}$ helped in the charge transfer and migration to coordinate the catalyst. Further, Swierk et al. systematically increased the presence of active sites in $\mathrm{Fe}_{x} \mathrm{Ni}_{1-x} \mathrm{OOH}$, by incorporating $\mathrm{Fe}^{3+}$ into $\mathrm{NiOOH} .{ }^{123}$ They found that the OER activity of $\mathrm{Fe}_{x} \mathrm{Ni}_{1-x} \mathrm{OOH}$ was faster as compared to $\mathrm{FeOOH}$ or $\mathrm{NiOOH}$ by using the impedance and activation energy measurement techniques. Also, the faradaic resistance of the monometallic catalyst was found to be 2-fold higher as compared to $\mathrm{Fe}_{x} \mathrm{Ni}_{1-x} \mathrm{OOH}$, whereas the activation energy was 3-fold higher. Besides, NiFe-LDH was found to contain $\mathrm{Ni}^{2+}$ and $\mathrm{Fe}^{3+}$ cations at low potential. ${ }^{124}$ At an elevated potential, $\mathrm{Ni}^{2+}$ is oxidized to $\mathrm{Ni}^{3+}$ with the formation of the $\gamma$ phase of $\mathrm{Ni}_{1-x} \mathrm{Fe}_{x} \mathrm{OOH}$, and $\mathrm{Fe}^{3+}$ remains unaltered (Fig. 10(a)). ${ }^{124} \mathrm{DFT}+\mathrm{U}$ studies also verified the phase transformation of NiFe-LDH to Fe-doped $\gamma$-NiOOH during the OER, which caused the shifting of the adsorption energies of the oxygen intermediates. Mostly, $\mathrm{Fe}^{3+}$ content increases the OER activity of NiFe-LDH by lowering the overpotential of $\gamma-\mathrm{Ni}_{1-x} \mathrm{Fe}_{x} \mathrm{OOH}$ as compared to $\mathrm{Ni}^{3+}$ in $\gamma-\mathrm{Ni}_{1-x} \mathrm{Fe}_{x} \mathrm{OOH}$ or $\gamma$-NiOOH (Fig. 10(b)). ${ }^{124}$

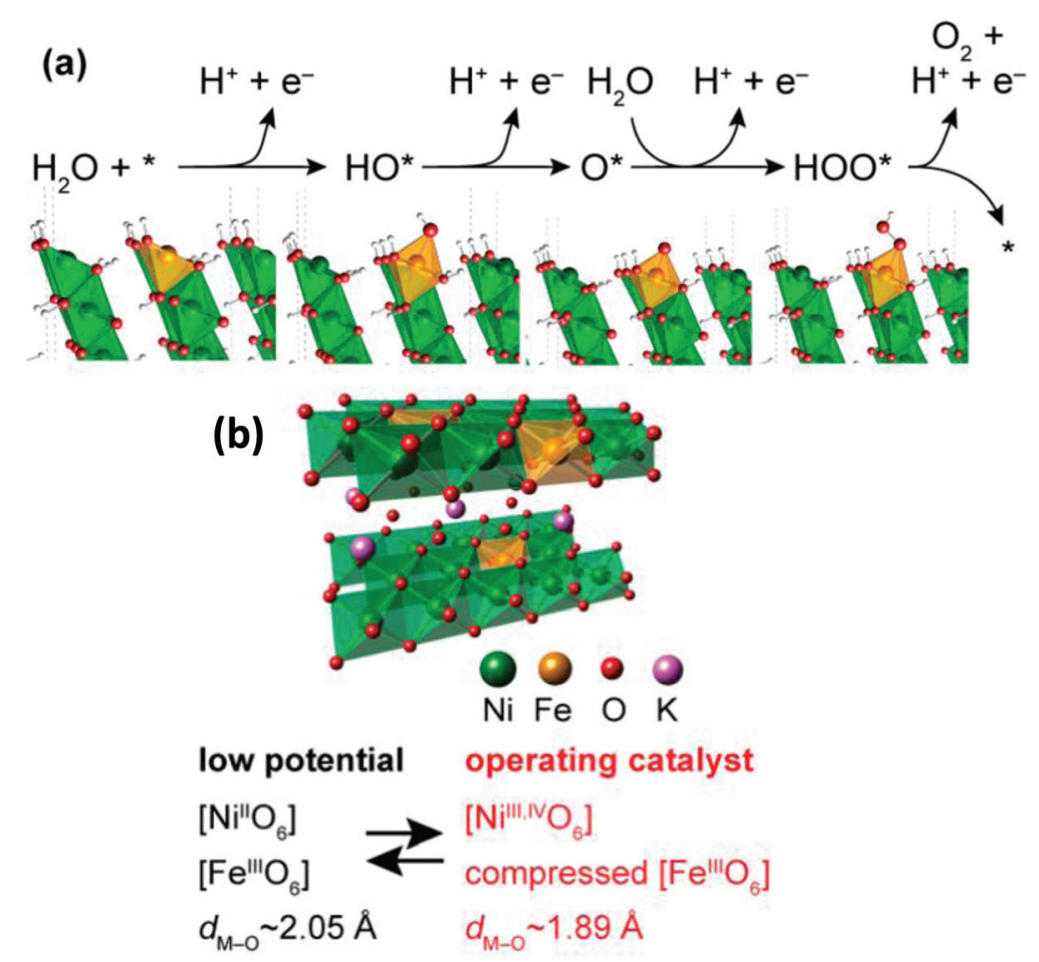

Fig. 10 (a) Representative OER route for $\mathrm{NiFe}-\mathrm{LDHs}$ and schematic models of the structural transformation of $\mathrm{NiFe}-\mathrm{LDH}$ into $\gamma$ - $\mathrm{Ni}_{1-x} \mathrm{Fe}_{x} \mathrm{OOH}$ through the OER potential, and the corresponding OER path for NiFe $\mathrm{LDHs}$ explains the involvement of $\mathrm{HO}^{*}, \mathrm{O}^{*}$, $\mathrm{OOH}^{*}$ intermediates and Fe-doped $\gamma$ - $\mathrm{NiOOH}$ as active sites. (b) Structure model of Fe-doped $\gamma$ - NiOOH. Reproduced with permission from ref. 124, Copyright 2015, American Chemical Society. 
As mentioned above, NiFe-based materials possess characteristic properties that drive the OER. Most importantly, the exposure of more active sites of NiFe-LDH with enhanced surface area is another approach to increasing the OER performance. Accordingly, Zhang et al. reported the morphological variation from the bulk NiFe-LDH to hollow microsphere (HMS) for better OER activities. ${ }^{84}$ Bulk LDH possesses the intrinsic characteristics of metal hydroxides, with low electrical conductivity that affects their catalytic properties. Song et al. exfoliated bulk NiFe-LDH to single layer NS by a liquid-phase exfoliation technique for superior OER activity. ${ }^{125}$ However, the complexity of the liquid phase exfoliation has drawn more attention towards the exfoliation free approach. Han et al. designed a single-layer NiFe-LDH NS by intercalating $\mathrm{MoO}_{4}{ }^{2-}$ ions through the hydrothermal reaction and achieved satisfactory OER performances. ${ }^{126}$ Nevertheless, the poor electrical conductivity of NiFe-LDH and the slow movement of the metal hydroxide layers $\left(\mathrm{Ni}(\mathrm{OH})_{2}, \mathrm{Fe}(\mathrm{OH})_{3}\right)$, drastically affected the mass transfer in the OER. Therefore, NiFe-LDHs are more prone to combining with highly conductive graphene as a support material for the enhancement of the electrical conductivity and the dispersion ability to promote accessibility towards electrochemically active metal centers for catalytic reactions. Following this approach, Long et al. reported the heterostructured $\mathrm{NiFe}-\mathrm{LDH} / \mathrm{rGO}$ hybrid by the electrostatic coupling of the positively charged NiFe-LDH NS with the nega- tively charged GO layers (Fig. 8(a)). ${ }^{112}$ As a result, this catalyst displayed outstanding OER activity in alkaline media, with the lowest overpotential of $0.195 \mathrm{~V}$ among the noble-metal-free catalysts, and a Tafel slope of $39 \mathrm{mV} \mathrm{dec}{ }^{-1}$, which is close to that of the $\mathrm{Ir} / \mathrm{C}$ catalyst. These outcomes are due to the homogeneous carbon network of $\mathrm{GO} / \mathrm{rGO}$ with large surface areas and the uniformly distributed active sites of NiFe-LDH, respectively.

Intercalated graphene has a tremendous role in enhancing the electrocatalysis of NiFe-LDHs. By utilizing the concept of intercalated graphene, Sasaki et al. designed the NiFe/GO superlattice heterostructure via the heteroassembly of NiFeLDH NS with GO NS by a coprecipitation method using anthraquinone-2-sulfonate (AQS) and hexamethylenetetramine (HMT) as the oxidizing and hydrolyzing agents, respectively, as described in the synthesis section (Fig. 9(a)). ${ }^{83}$ The morphology of the NiFe/rGO superlattice shows the periodic stacking of NiFe-LDH with GO/rGO, as revealed by the high-resolution TEM/HRTEM image (Fig. 11(a and b)). The graphene, especially for the more conductive rGO layers intercalated into the NiFe-LDHs, is useful for the improvement of electrocatalytic activities of the NiFe-LDHs/rGO superlattice (Fig. 11(c and $\mathrm{d}$ )). It displayed a low overpotential of $0.21 \mathrm{~V}$ with a Tafel slope of $40 \mathrm{mV}$ per decade, a bit higher than that reported by Long et al., i.e., NiFe-LDH/rGO (0.195 V, $\left.40 \mathrm{mV} \mathrm{dec}^{-1}\right) .{ }^{112}$ Overall, the improved OER performances of NiFe-LDH/rGO
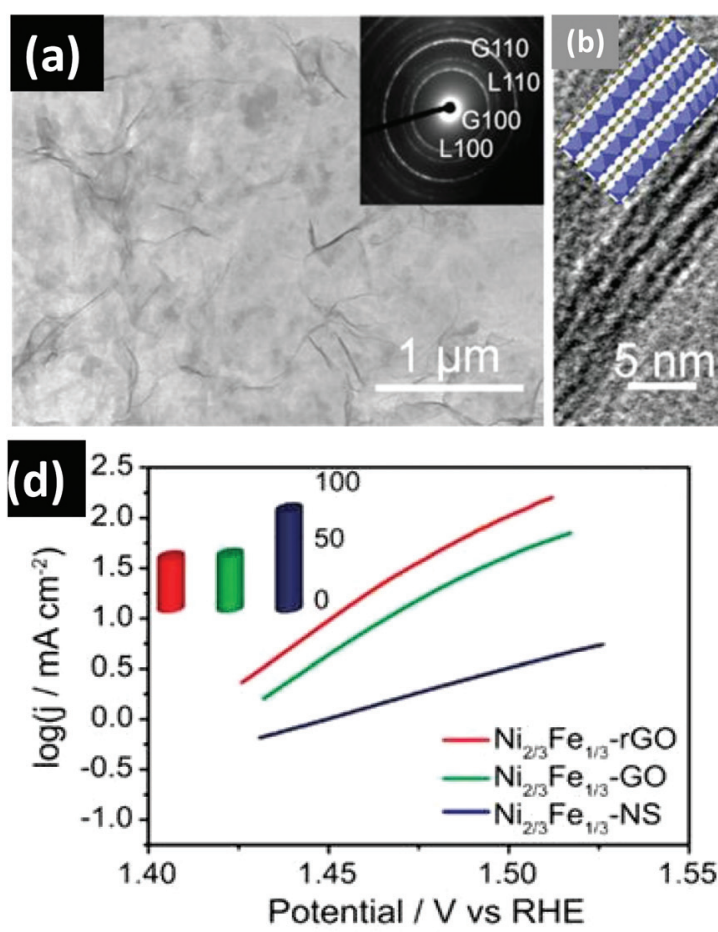
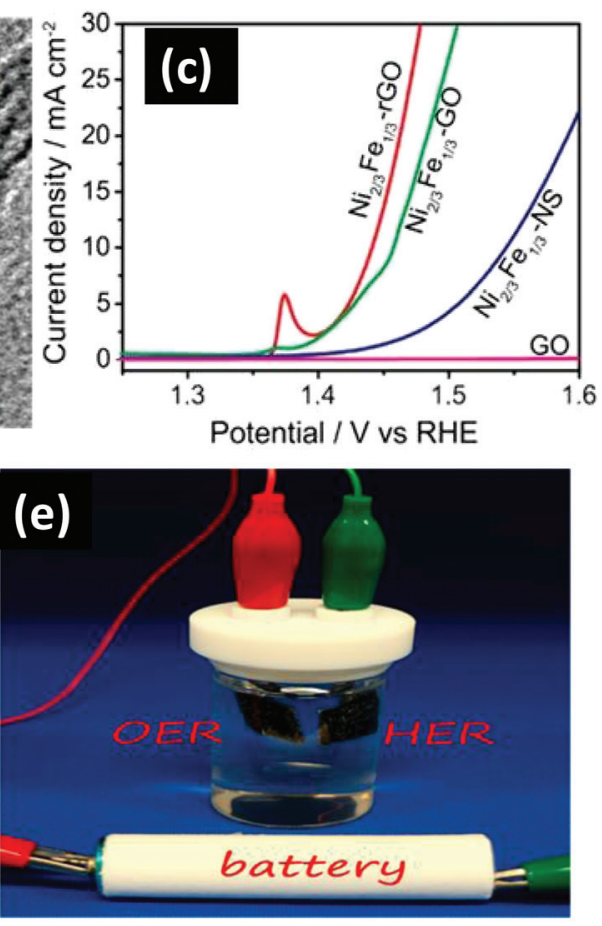

Fig. 11 (a) TEM image of $\mathrm{Ni}_{2 / 3} \mathrm{Fe}_{1 / 3}-\mathrm{NS} / \mathrm{GO}$ composites. (b) HRTEM image of $\mathrm{Ni}_{2 / 3} \mathrm{Fe}_{1 / 3}-\mathrm{NS}$ and GO composites. (c) OER activity of $\mathrm{Ni}_{2 / 3} \mathrm{Fe} \mathrm{I}_{1 / 3}-\mathrm{NS}$, $\mathrm{Ni}_{2 / 3} \mathrm{Fe}_{1 / 3}-\mathrm{GO}$, and $\mathrm{Ni}_{2 / 3} \mathrm{Fe}_{1 / 3}-\mathrm{rGO}$ with IR-compensation. (d) Tafel plot of $\mathrm{Ni}_{2 / 3} \mathrm{Fe}_{1 / 3}-\mathrm{NS}, \mathrm{Ni}_{2 / 3} \mathrm{Fe}_{1 / 3}-\mathrm{GO}$, and $\mathrm{Ni}_{2 / 3} \mathrm{Fe}_{1 / 3}-\mathrm{rGO}$. (e) Water-splitting electrolytic cell power-driven by 1.5 V AA battery. Adapted with permission. ${ }^{83}$ Copyright 2015, American Chemical Society. 
have been attributed to the intercalation of $\mathrm{GO} / \mathrm{rGO}$, which restricts the self-aggregation of $\mathrm{NiFe}-\mathrm{LDH} \mathrm{NS}$ and further enhances the exposure of more electrochemical active sites of $\mathrm{NiFe}-\mathrm{LDH}$ NS. Also, the face-to-face contact between NiFe-LDH and GO exerts stronger interfacial interactions for the electrocatalytic OER process. However, the authors also established an electrolyzer cell fueled by a battery of $1.5 \mathrm{~V}$ AA for overall water splitting (Fig. 11(e)). The strategy established by Sasaki's group was further applied to NiMn-LDHs NS for superior electrochemical performance. ${ }^{127}$

More importantly, the incorporation of topological defects by the removal of heteroatoms on the graphene surface is of great importance in accelerating the electrocatalytic water splitting reactions. This defective graphene (named DG) has a greater affinity for the anchoring of transition metal atoms through strong $\pi-\pi$ bonding interactions and acts as the active sites for the overall water splitting and oxygen reduction reactions. $^{128}$ Jia et al. designed a heterostructured NiFe-LDH NS/ DG by the electrostatic coupling of NiFe-LDH NS onto defective graphene and used it as a bifunctional electrocatalyst for overall water splitting (Fig. 12(a)). ${ }^{92}$ In particular, the OER catalytic activity of NiFe-LDH NS/DG in alkaline solution was enhanced with a small Tafel slope of $52 \mathrm{mV} \mathrm{dec}^{-1}$ and the overpotential was reduced to $210 \mathrm{mV}$ at the current density $J=$ $10 \mathrm{~mA} \mathrm{~cm} \mathrm{~cm}^{-2}$ (Fig. 12(b-d)). NiFe-LDH-NS/DG also performed well in the HER with a Tafel slope of $110 \mathrm{mV} \mathrm{dec}^{-1}$ and a reduced overpotential of $115 \mathrm{mV}$ at the current density of $20 \mathrm{~mA} \mathrm{~cm}^{-2}$ in $1 \mathrm{M} \mathrm{KOH}$ (Fig. 12(e and f)). Moreover, NiFeLDH-NS/DG showed excellent stability towards the OER (Fig. 12(d)) and HER (Fig. 12(g)) in $1 \mathrm{M} \mathrm{KOH}$ electrolyte. The advantages of coupling NiFe-LDH-NS/DG for outstanding overall water splitting performances are as follows: (i) DG with
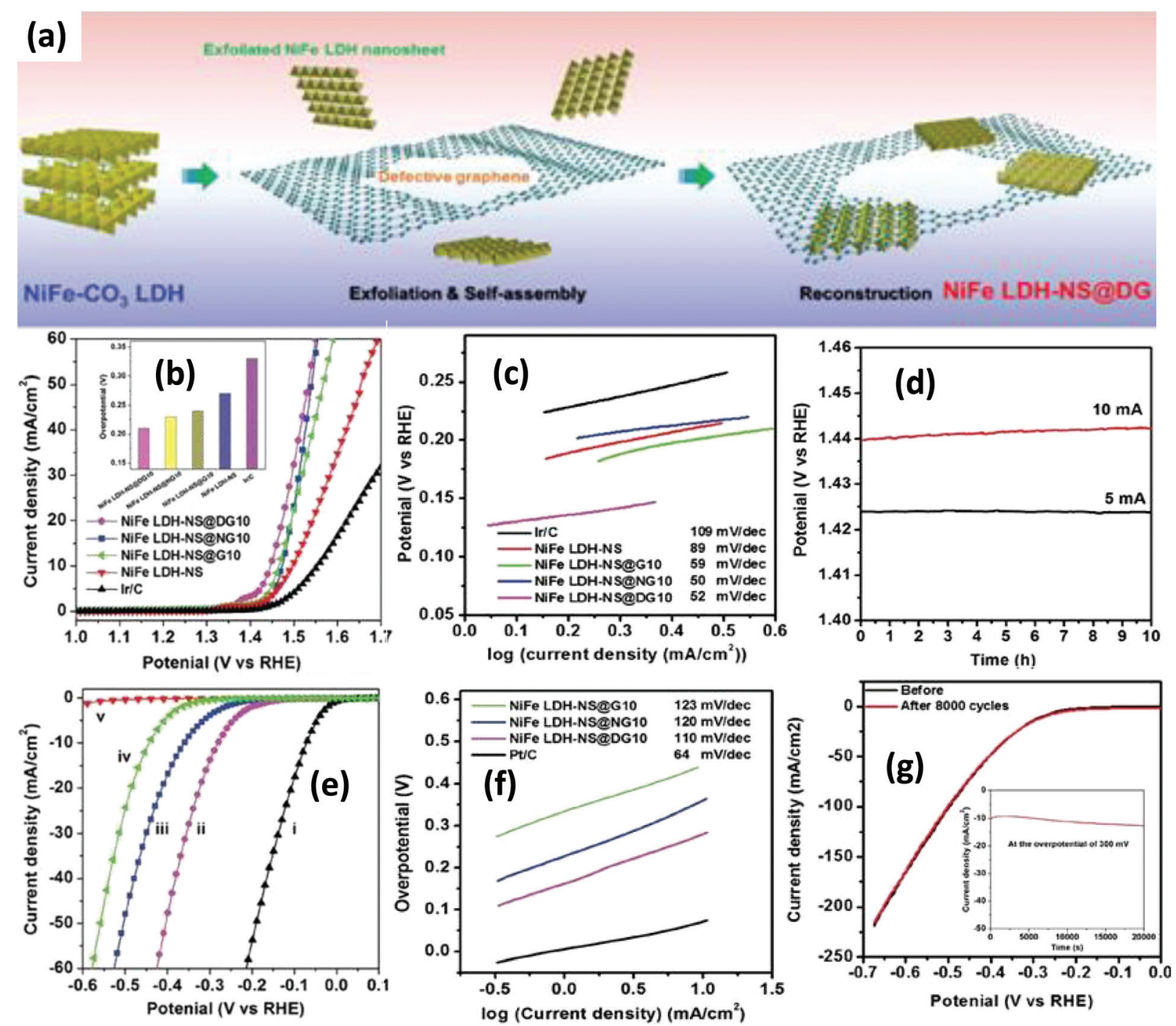

Fig. 12 (a) Illustration of the synthetic route to the NiFe-LDH-NS/DG hybrid. (b) OER LSV curves of the NiFe-LDH-NS/DG series and related materials in $1 \mathrm{M} \mathrm{KOH}$. (c) OER Tafel plots. (d) Stability testing of the NiFe LDH-NS@DG10. (e) HER LSV plots (f) HER Tafel plots. (g) Polarization versus chronoamperometric curves of the NiFe LDH-NS@DG10. Reproduced and modified from ref. 92, Copyright 2017, Wiley VCH. 
defective sites provides superior conductivity and high surface area that facilitates the formation of a heterostructure with NiFe-LDH NS for maximum direct interfacial interaction. (ii) The heterostructure formation causes interfacial contractions between Ni/Fe atoms and DG, which speed up the electronic transportation and shorten the diffusion length. (iii) The good dispersion of NiFe LDH NS on DG exposes all active sites of NiFe-LDH for electrocatalytic advantages.

The NiFe-LDH/graphene heterostructures discussed above are promising materials for electrocatalytic water oxidation. Nevertheless, the hydroxides reportedly show inferior HER activity as compared to phosphides and chalcogenide materials. In contrast, the $\mathrm{LDH} / \mathrm{MoS}_{2}$ superlattice has received much attention because the 1T-phase $\mathrm{MoS}_{2}$ not only behaves as a conductive framework imparting conductivity to the heterostructure, it also shows HER activity similar to commercial Pt/C. Motivated by this, Xiong et al. prepared the $\mathrm{MoS}_{2} /$ NiFe-LDH superlattice via a solution-phase assembly by restacking the two oppositely charged NS (Fig. 13(a)). ${ }^{110}$ When $\mathrm{MoS}_{2}$ and LDH NS were restacked in a layer-by-layer assembly, significant radical charge distribution by electron accumulation at the interfacial area and electron transfer among adjacent monolayers occurred, which synergistically promoted the adsorption of intermediates both in the OER and HER (Fig. 13(b)). The transfer of electrons from the LDH to $\mathrm{MoS}_{2}$ was $0.45 \AA^{-2}$, which revealed the strong electronic coupling between metallic $\mathrm{MoS}_{2}$ and NiFe-LDH NS as compared to that for $\mathrm{LDH} /$ graphene (named $\mathrm{LDH} / \mathrm{G}$ ) and $\mathrm{MoS}_{2} /$ graphene $\left(\mathrm{MoS}_{2} /\right.$ $\mathrm{G})$ superlattices. The modulated electronic environment of $\mathrm{MoS}_{2} / \mathrm{LDH}$ increased the adsorption between the intermediates and electrocatalyst. Specifically, the localized electron or hole accumulation at the basal edges of $1 \mathrm{~T} \mathrm{MoS}_{2}$ or the LDH NS counterparts resulted in the improved affinity for $\mathrm{H}^{+}$or $\mathrm{OH}^{-}$, respectively, which are favorable for efficient OER and HER. This $\mathrm{MoS}_{2} / \mathrm{LDH}$ superlattice acts as a bifunctional electrocatalyst, displaying enhanced activity as compared to the other two superlattices (Fig. 13(c and d)). In particular, $\mathrm{MoS}_{2} /$ $\mathrm{LDH}$ exhibited superior HER performance to the $\mathrm{MoS}_{2} /$ graphene composite and unilamellar $\mathrm{MoS}_{2}$ NS. Most importantly, the rating of $\mathrm{MoS}_{2} / \mathrm{LDH}$ as a bifunctional catalyst for the overall water splitting even surpasses the $\mathrm{RuO}_{2}(+)|| \mathrm{Pt} / \mathrm{C}(-)$ couple as shown in Fig. 13(e). The molecular-level modulation of the heterointerface between the $2 \mathrm{D} \mathrm{LDH}$ and other unilamellar NS could be of great promise in advanced electrocatalysis.

As mentioned previously, the superlattice structure can effectively modulate the electronic environment of a material. To further optimize the electronic coupling as well as the adsorption energy, the presence of 3D porosity in NiFe-LDH/ rGO could be another strategy for enhancing the catalytic activities of nanomaterials. Porosity increases the active sites on the catalyst surface as well as mass transfer. ${ }^{129}$ Zhan et al. reported a porous nanostructure of NiFe-LDH/rGO via a facile two-step process involving the reverse micelle solvothermal method and chemical reduction (Fig. 14(a)). The porosity of the NiFe-LDH/rGO nanospheres is due to the aggregation of the $\mathrm{LDH}$ hexagonal plates to produce numerous defective microspheres owing to the reverse micelle effect caused by the role of surfactants as templates..$^{93}$ Broken GO nanoplates are then attached to the LDH surface, cross-linking it with their oxygen-containing functional groups to generate a rough porous surface as evident from TEM and HRTEM images in Fig. 14(b and c). ${ }^{93}$ The NiFe-LDH/rGO are highly active electrocatalysts for the OER and ORR, providing excellent OER catalytic activity with a low onset overpotential of $250 \mathrm{mV}$ and a Tafel slope of $91 \mathrm{mV}$ per decade (Fig. 14(d and e)). These enhanced dual catalytic tendencies may have arisen from the surfactant-induced porous microstructure and the sturdy bonding effect of rGO with NiFe-LDH, which are favorable for improved conductivity, more exposure of active sites, and both mass and charge transport of oxygen towards electrocatalytic reactions. Alternatively, the conductive graphene support for the FeNi-LDH generates a 3D framework that is interconnected with the catalytically exposed sites and adequate micro/macroporous channels for fast electron transportation, which facilitate the gas molecule and electrolyte diffusion for the OER. Zhang et al. used a single-step hydrothermal method to introduce NiFe-LDHs into the 3D rGO framework, with conductive CNT (FeNi-GO/rGO LDH) as an additive for further application in the OER. ${ }^{117}$ The most outstanding OER performance of NiFe-LDH/rGO had a Tafel slope of $39 \mathrm{mV} \mathrm{dec}^{-1}$ and overpotential of $206 \mathrm{mV}$. In this illustration, FeNi-rGO/LDH demonstrated greater OER performance, possibly due to the nucleation and growth strategy of NiFe-LDH on GO/rGO and further hybridization through the interlayer of NiFe- $\mathrm{LDH}$, which facilitated the disclosure of the active sites in the catalyst, thus increasing the charge transfer kinetics through the rGO layers. By forming $\mathrm{NiFe}-\mathrm{LDH} / \mathrm{rGO}$, tuning the intrinsic properties of the NiFe-LDH layers triggered the incrementally exposed surface of NiFe hydroxide, resulting in an inimitable layered structure that could effectively encourage the OER performance.

For a better deposition of NiFe-LDH on the graphene surface, the open porous structures of the 3D graphene frameworks having higher conductivity, surface defects, flexibility, and lightweight are desirable factors. As discussed in the growth strategy, Shi et al. described a double-step approach to designing a freestanding NiFe-LDH/3DErGO electrode (Fig. 5(c)). ${ }^{78}$ The co-operative result was that NiFe-LDHs and 3D-ErGO substrates formed an exceptional OER catalyst with a

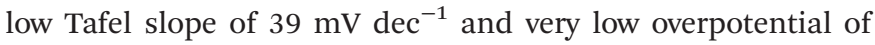
$259 \mathrm{mV}$ at $10.0 \mathrm{~mA} \mathrm{~cm}{ }^{-2}$. The crucial role of Fe in the NiFe LDHs phase was observed with a drastic reduction of the Tafel slope and overpotential with the doping content of $\mathrm{Fe}$ (Fig. 14(f-i)).

Recently, Feng et al. assembled a strong system of 3D ternary electrodes by the in situ growth of vertically aligned $\mathrm{Co}_{0.85} \mathrm{Se}$ NS on delaminated graphene foil, followed by the growth of NiFe-LDHs by a simple hydrothermal reaction, which displayed excellent performance in the OER with a $250 \mathrm{~mA} \mathrm{~cm}{ }^{-2}$ current density at a small potential of $1.51 \mathrm{~V}^{130}$ Heterogeneous atom doping is a significant strategy for enhan- 
(a)

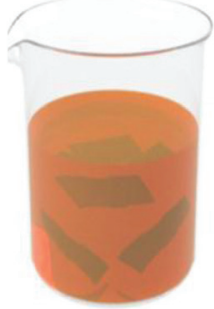

Negatively charged A nanosheets

(b)

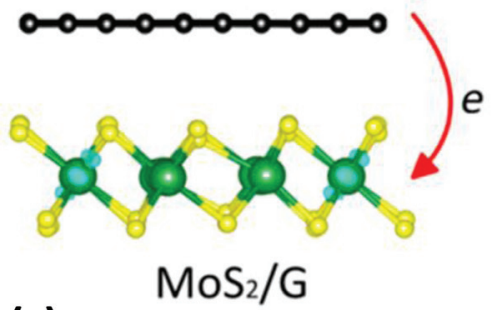

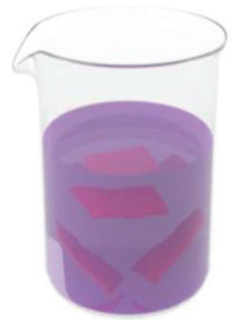

Positively charged $B$ nanosheets

$0.15 / \AA^{-2}$

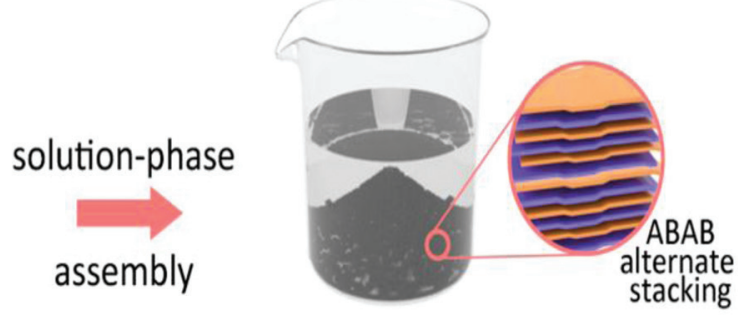

Flocculated superlattice-like structure

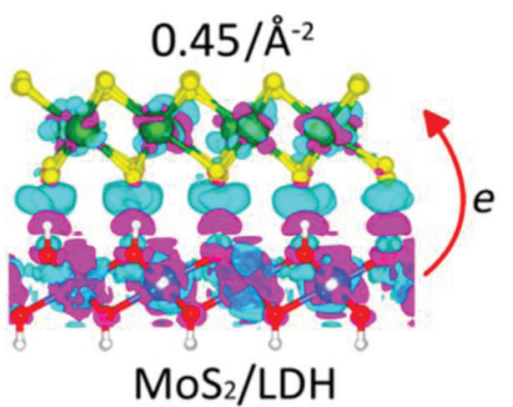

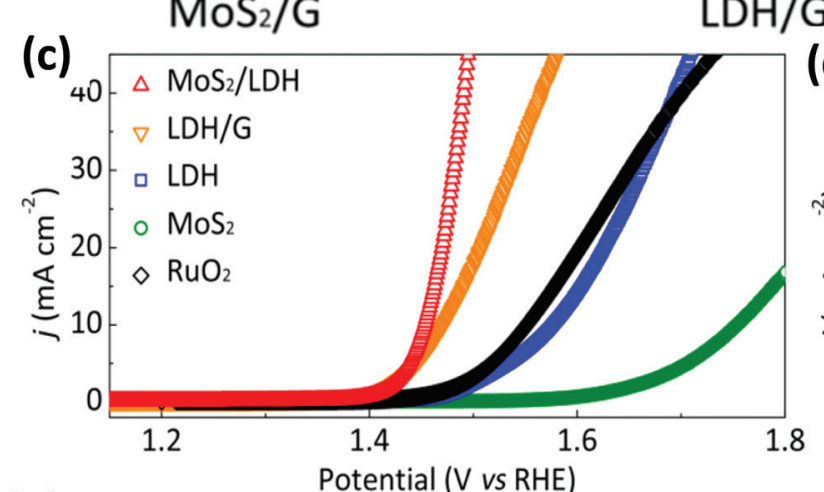

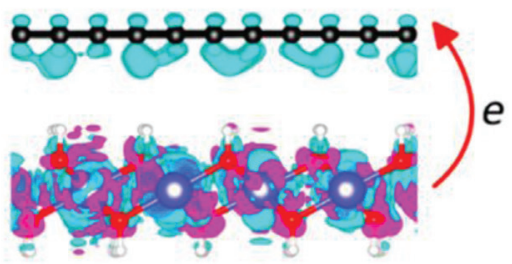

$\mathrm{LDH} / \mathrm{G}$

(e)
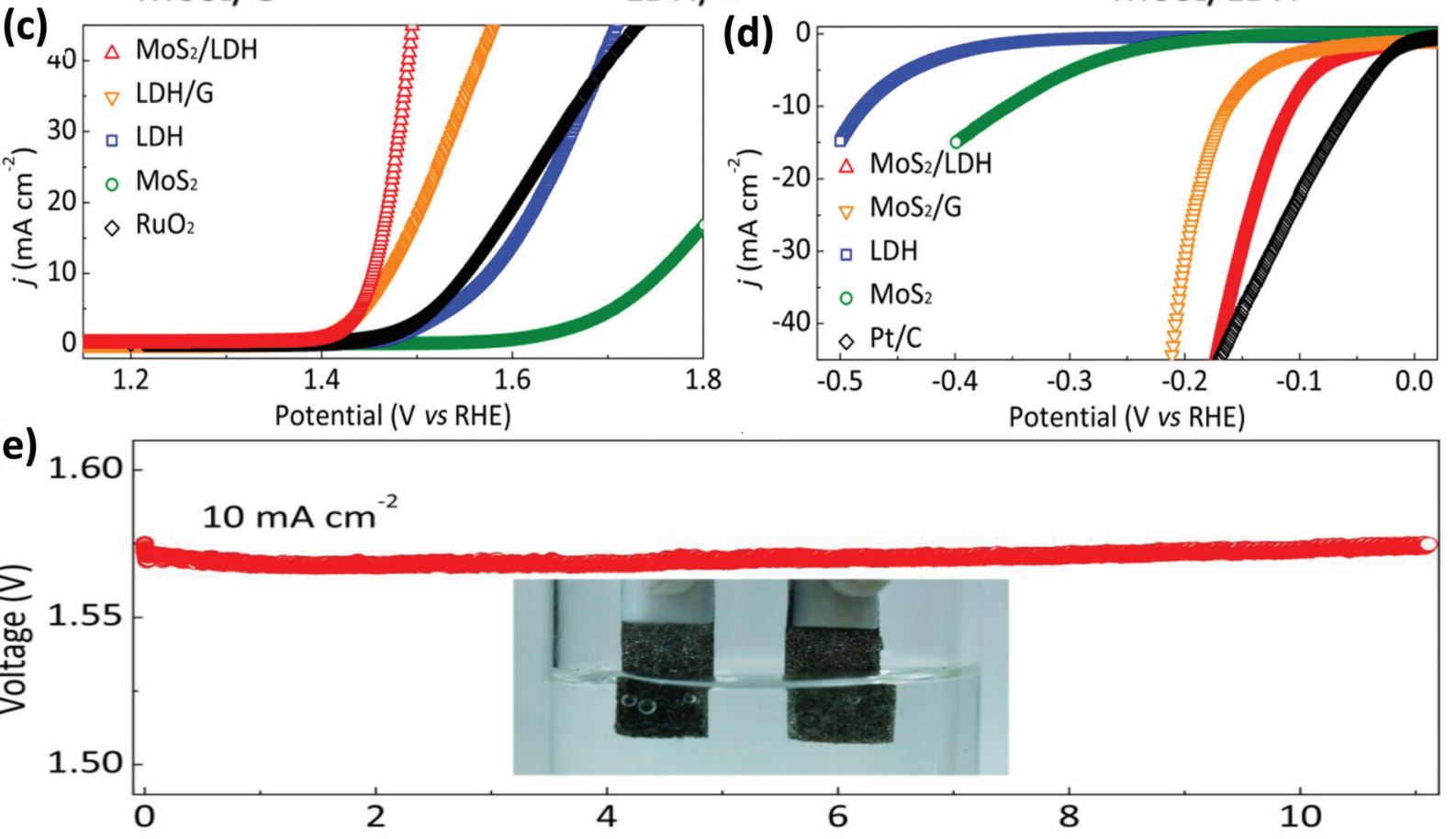

Potential (V vs RHE)

\section{$0.0042 / \AA^{-2}$}

HE)

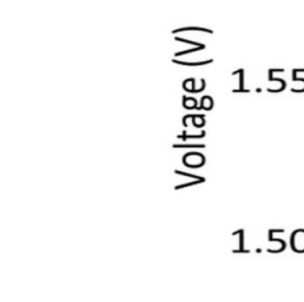

4 Time (h)

Fig. 13 (a) Schematic of the fabrication of the superlattice by the solution-phase assembly. (b) Bilayer charge distribution by the Bader charge analysis of the $\mathrm{MoS}_{2} / \mathrm{G}, \mathrm{LDH} / \mathrm{G}$ and $\mathrm{MoS}_{2} / \mathrm{LDH}$ superlattices. Electrocatalytic performance of $\mathrm{MoS}_{2} / \mathrm{LDH}$ and related material for (c) the OER and (d) HER. (e) Evaluation of the stability and overall water splitting activity by the $\mathrm{MoS}_{2} / \mathrm{LDH}$ superlattice as the bifunctional electrocatalyst. Adapted with permission. ${ }^{110}$ Copyright 2019, American Chemical Society.

cing the properties of graphene-related nanomaterials, owing to the positive co-operative effect among heteroatom dopants, carbon atoms and transition metals. ${ }^{131,132}$ Zhang and Wei et al. designed $\mathrm{N}$-doped graphene frameworks with spatially confined hybridization with nano NiFe-LDHs (nNiFe-LDH/ NGF). ${ }^{106}$ In this work, the tremendous catalytic activities in the OER were proved by the remarkable lowering of the overpotential $\left.(\sim 337 \mathrm{mV} \text { at } 10 \mathrm{~mA} \mathrm{~cm})^{-2}\right)$ and a calculated Tafel slope of $45 \mathrm{mV} \mathrm{dec}{ }^{-1}$ with prolonged durability, which indicate the significance of the work for better electrocatalysis. This work is of importance because of the electron donor properties of $\mathrm{N}$-doping and $\mathrm{N}$-possessing active sites to trigger 

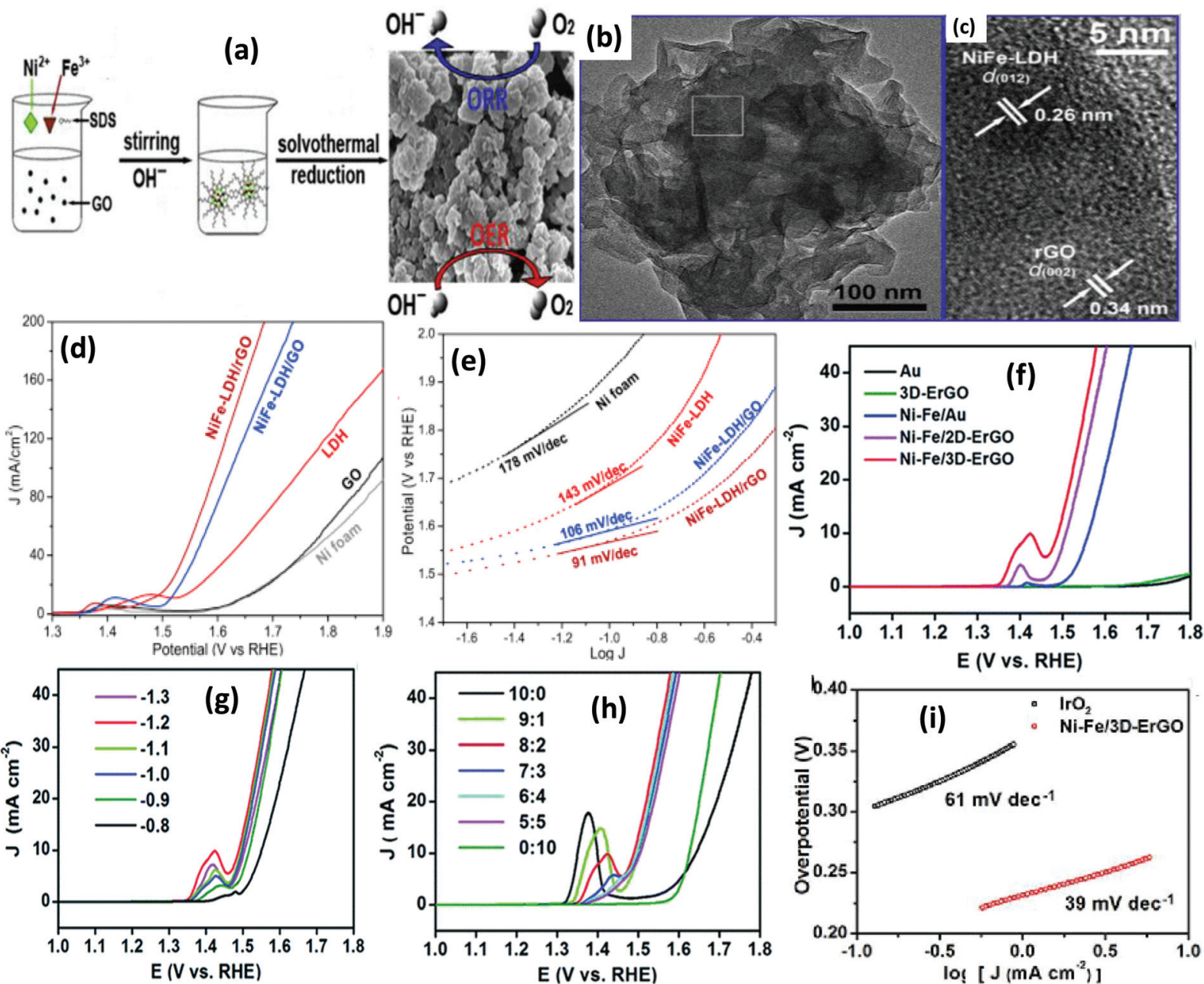

Fig. 14 (a) Synthesis of NiFe-LDH/rGO composites. (b) TEM images of the NiFe-LDH/rGO composites. (c) HRTEM image of the NiFe-LDH/rGO composite. (d) OER activity on Ni foam, GO, LDH, NiFe-LDH/GO, NiFe-LDH/rGO. (e) Tafel plots of Ni foam, GO, LDH, NiFe-LDH/GO and NiFe-LDH/ rGO electrodes. Adapted with permission. ${ }^{93}$ Copyright 2016, Elsevier. (f) LSV curves of Au, 3D-ErGO, NiFe/Au, NiFe/2D-ErGO, and NiFe/3DErGO electrodes. (g) LSV curves of Ni-Fe/3D-ErGO electrodes prepared at the NiFe deposition potential ranging between -0.8 and $-1.3 \mathrm{~V}$. (h) LSV plot of the NiFe/3D-ErGO from the variation of Ni/Fe ratios in the precursor solution. (i) Tafel plots of NiFe/3D-ErGO and IrO ${ }_{2}{ }^{78}$ Adapted with permission from the Royal Society of Chemistry.

carbon atoms in graphene material for the creation of a large number of defects or active sites to augment the electrocatalytic activities.

The 3D porous structured graphene, with high surface area and porosity and the homogeneous distribution of the porosity in support materials, created a contact surface between the reactant molecules and electrochemically active centers for faster mass transfer. Along with the in-depth investigation into the oxygen reduction reaction (ORR) catalysts, layered structures with pores and interconnectivity would certainly increase the density and contact of extremely sensitive sites. ${ }^{133}$ Therefore, the design of 3D graphene aerogels is of great importance to the optimization of the mass transfer as well as the exposure of the extremely sensitive active sites of a catalyst. Very recently, Manna et al. designed nanostructured NiFe-LDH decorated over the N-doped entangled graphene framework (NEGF), by an in situ solvothermal and freeze-drying method at low temperature and high vacuum pressure. ${ }^{76}$ The freeze-drying method significantly contributes to the formation of a stable entangled graphene network as shown in Fig. 15(a). The synergistic effect of NEGF in the strong coupling with NiFe-LDH facilitated a drastic reduction in the electrocatalytic OER overpotential of NiFe-LDH/NEGF to $290 \mathrm{mV}$, with a small Tafel slope of

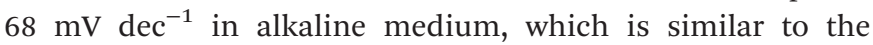
recent record (Fig. 15(b and c)). ${ }^{76}$ In EC water splitting, the role of graphene in the NiFe-LDH/graphene system is to optimize the mass transfer process by participating as a support material through the 2D-conductive substrate, the open porous structure of the $3 \mathrm{D}$ graphene frameworks, the $3 \mathrm{D}$ porous entangled structure or free-standing structure, and the $3 \mathrm{D}$ defective graphene support, respectively. 

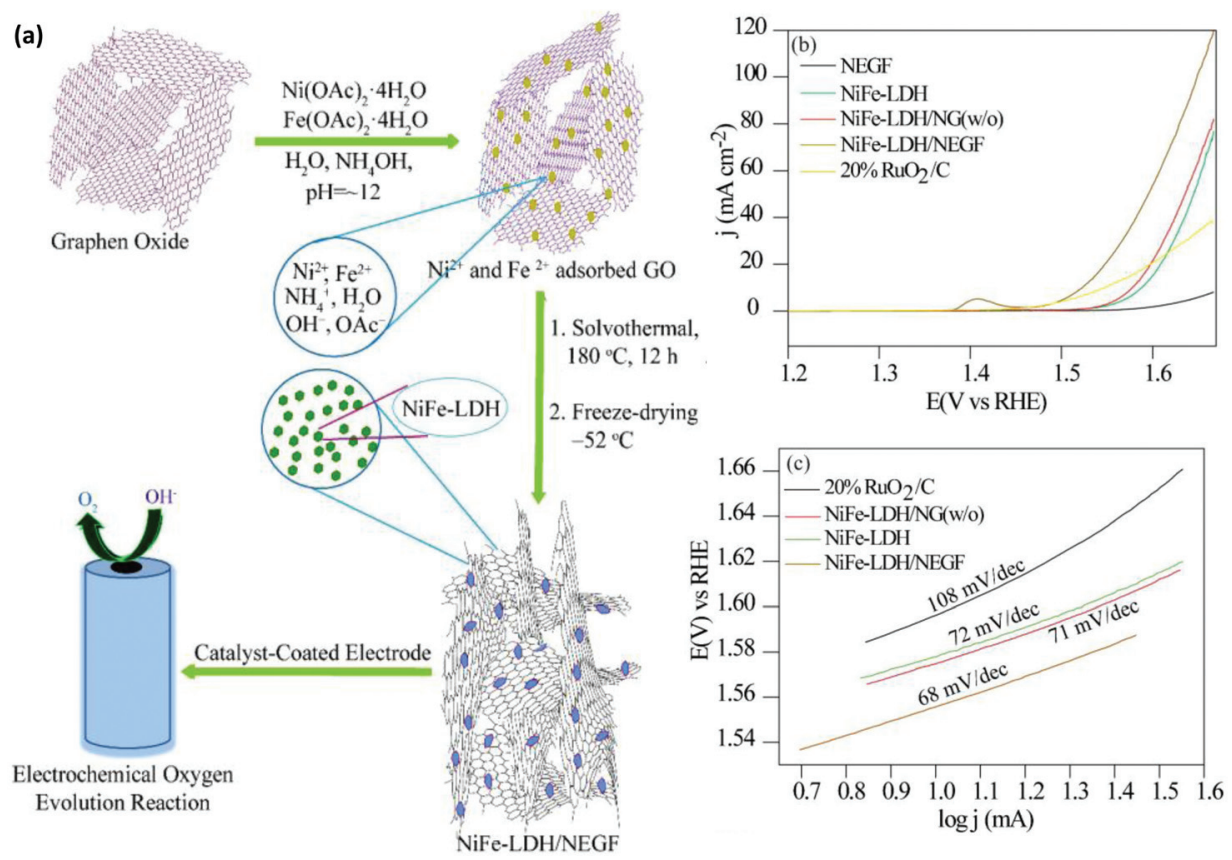

Fig. 15 (a) A pictorial depiction of the synthetic steps involved in NiFe-LDH/NEGF and the corresponding OER activity. (b) Polarization activity of NEGF, NiFe-LDH, NiFe-LDH/NG (w/o), NiFe-LDH/NEGF, and 20\%RuO $/$ C. (c) Tafel plots of NiFe-LDH/NEGF, NiFe-LDH, NiFe-LDH/NG (w/o), and 20\% $\mathrm{RuO}_{2} / \mathrm{C}^{76}$ Adapted with permission from the Royal Society of Chemistry.

\section{Photoelectrochemical (PEC) water splitting}

PEC cells are capable of converting direct energy from sunlight into chemical energy. Inside the water splitting reaction, $\mathrm{H}_{2} \mathrm{O}$ is oxidized by minority charge carrier holes at the photoanode, releasing electrons and protons into the solution, with the reduction reaction occurring at the cathode, liberating $\mathrm{H}_{2}$. PEC water splitting into $\mathrm{H}_{2}$ and $\mathrm{O}_{2}$ by using solar energy combined with a solar energy collector and water electrolysis in a solitary photoelectrode, which could perform both light absorption and the water splitting reaction. Semiconductors are generally used as photoelectrodes in PEC water splitting reactions. When an n-type semiconductor having a higher chemical potential (calculated by Fermi level alignment) is in contact with the electrolyte, the flow of electrons takes place from the semiconductor to the solution unless the chemical equilibrium is maintained in the solution (Fig. 16(a and b)). In this method, charge transport generates positive depletion or space-charge-region correlating interfacial electric fields that stabilize the difference in potentials among the electrodes and electrolyte solution. However, an unsymmetrical population of carrier charge is established between the VB and $\mathrm{CB}$ edge of semiconductors with the irradiation of quantum of energy greater than the bandgap energy of the semiconductor (Fig. 16(c)). This causes the formation of a quasi-Fermi zone between the electrons of the $\mathrm{CB}\left(E_{\mathrm{F}}, \mathrm{n}\right)$ and the holes of the $\mathrm{VB}$ $\left(E_{\mathrm{F}}, \mathrm{p}\right)$. This potential difference across the Fermi levels of the junction, the inbuilt photovoltage $\left(E_{\mathrm{pv}}\right)$, is the supreme force for the water oxidation reaction (Fig. 16(c)). Nevertheless, an external potential bias $\left(E_{\text {app }}\right)$ is still needed for superior charge separation by varying the inner electric field and mass transport by an external circuit through the transportation of electrons formed in the anodic reaction to the cathode. However, the external potential bias required in PEC is much smaller than that of the EC cell.

The NiFe-LDH/graphene hybrid was employed to facilitate the separation of exciton pairs and thus advance the photocurrent density of PEC electrodes with improved kinetics of the $\mathrm{O}_{2}$ evolution reactions. The synergistic effect of $\mathrm{N}$-doping and oxygen vacancies can contribute to the superior catalytic activity and stability towards PEC water oxidation. ${ }^{60}$ As discussed in the electrocatalytic OER section, the combination of the $\mathrm{N}$-doping and $3 \mathrm{D}$ porous structure could be another weapon for effective charge separations in the NiFe-LDH/graphene nanohybrid. Accordingly, Hou et al. designed 3D hybrid aerogels by mixing $\mathrm{N}$-deficient porous g- $\mathrm{C}_{3} \mathrm{~N}_{4}$ NS (DPCN), $\mathrm{N}$-doped GO/rGO (NRGO), and NiFe-LDH NS via the hydrothermal and freeze-drying methods (Fig. 17(a-d)). ${ }^{60}$ The morphology of DPCN/NRGO/NiFe-LDH revealed the interconnected $3 \mathrm{D}$ hierarchical structure and the elemental composition was verified by energy-dispersive X-ray (EDX) spectroscopy. The spatial separation of photogenerated exciton pairs and the unique $3 \mathrm{D}$ porous structures with micro-pores favoring mass transfer are the reason this ternary DPCN/NRGO/NiFe-LDH catalyst showed a higher photocurrent density of $162.3 \mu \mathrm{A}$ $\mathrm{cm}^{-2}$ at $1.4 \mathrm{~V}$ with unusual photostability after more than $10 \mathrm{~h}$ under AM 1.5 G exposure (Fig. 17(e and f)). ${ }^{60}$ Moreover, the charge transfer mechanism revealed that the photogenerated 


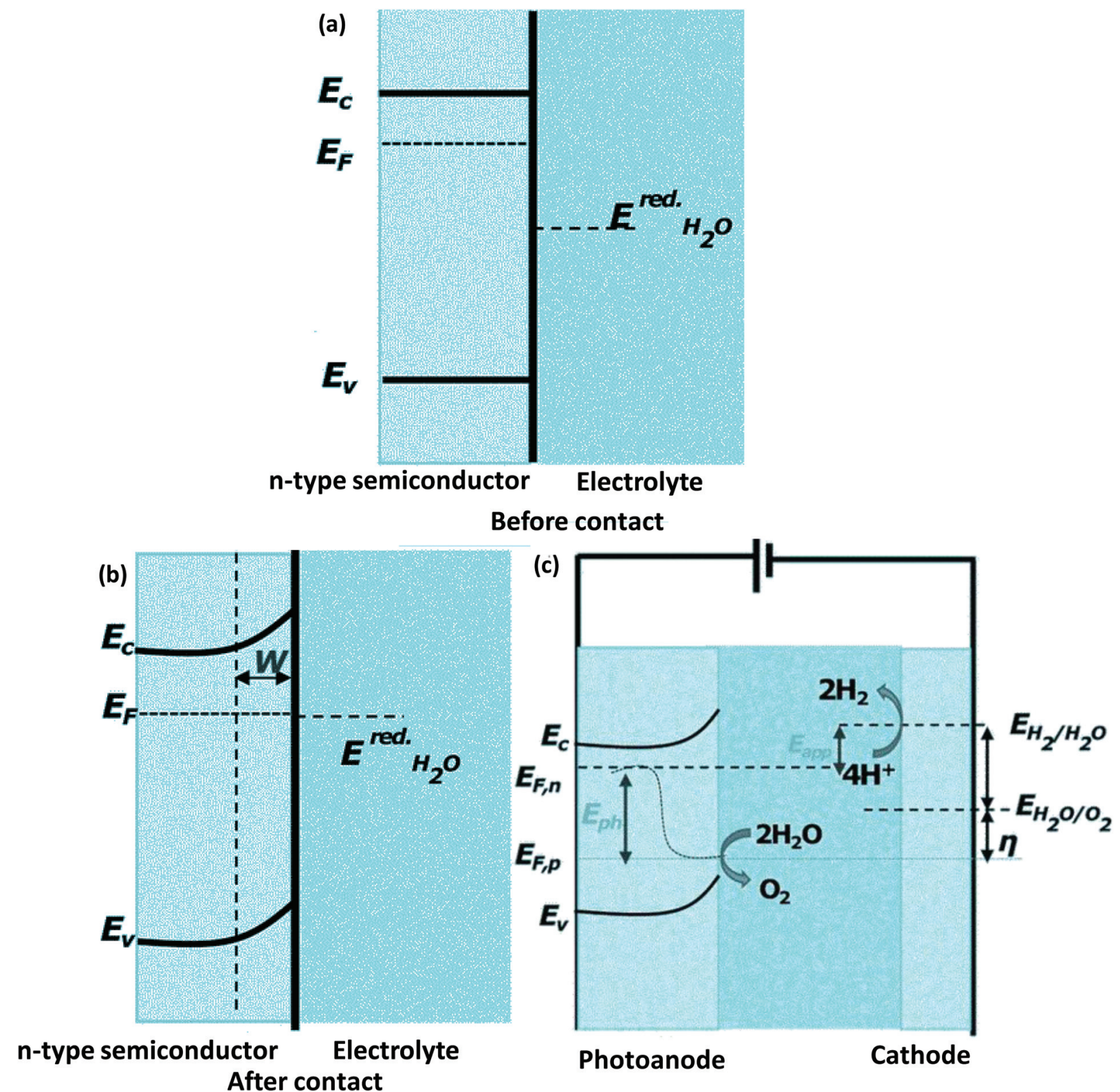

Fig. 16 Schematic representation of (a) the interface of the semiconductor and electrolyte before contact. (b) Equilibration of the interface of the semiconductor-electrolyte after contact. (c) $\mathrm{PEC}$ water splitting mechanism under irradiation; $E_{\mathrm{c}}=\mathrm{CB}$ edge potential, $E_{\mathrm{V}}=\mathrm{VB}$ edge potential, $E_{\mathrm{F}}=$ Fermi edge, $E_{\mathrm{app}}=$ applied potential, $E_{\mathrm{pv}}=$ photovoltage, $W=$ depletion area, $E^{\mathrm{red}} \mathrm{H}_{2} \mathrm{O}=$ redox potential of $\mathrm{H}_{2} \mathrm{O}$ or the electrolytic solution.

CB electrons of DPCN easily migrated towards the 3D NRGO network. Consequently, the 3D NRGO behaved as an electronconducting transport "highway" that further drifted the electrons toward the current collector. Meanwhile, hole transfer takes place from the VB of DPCN to the NiFe-LDH surface of the NRGO interlayer, which facilitates water oxidation reactions. This process justified the synergistic catalytic effect by constituent semiconductors towards the high-performance PEC water oxidation activity.

Generally, the physicochemical properties of LDHs can be exploited by constructing different hierarchical structures as inspired by nature. The rational design of hierarchical nano- structures such as core-shell, NAs, and nano-pyramids could be an efficient approach to enhancing the PEC water splitting. Hierarchical nanostructures of NiFe-LDH/GO could form $3 \mathrm{D}$ channels for rapid electron and hole separations. Han et al. designed the $\mathrm{BiVO}_{4} / \mathrm{rGO} / \mathrm{NiFe}$ hydroxide photoanode that encompasses hierarchical nanostructures by using a facile annelation-photoreduction-electrophoretic deposition method. ${ }^{88}$ The morphological features of this ternary nanocomposite reveal a nanopyramid pattern with the NAs of $\mathrm{BiVO}_{4}$ wrapped in the interlayer mixing of graphene NS and amorphous NiFe hydroxides, achieving the highest current density of $1.30 \mathrm{~mA} \mathrm{~cm}{ }^{-2}$ at $1.23 \mathrm{~V}$. The boost in current 


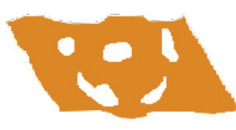

DPCN

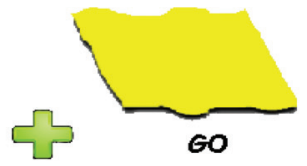

(a)

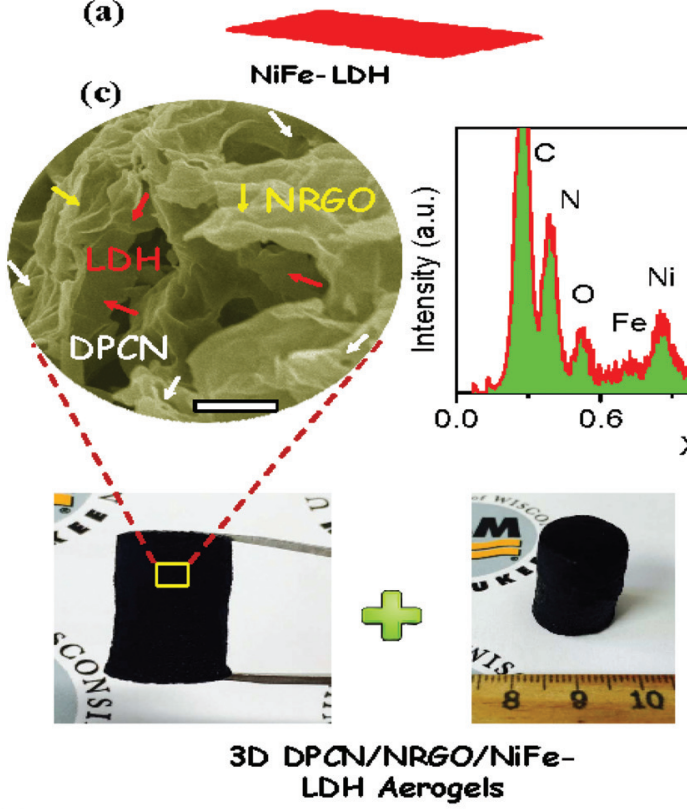

(e)

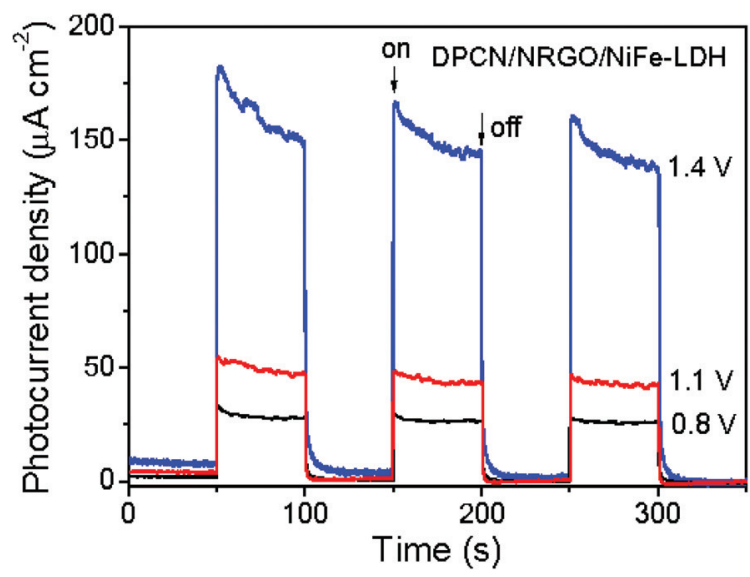

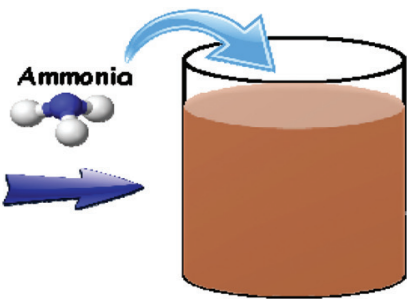

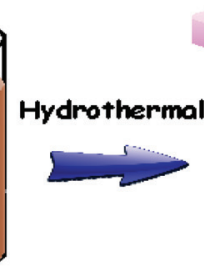

(d)

$\mathrm{Si}$

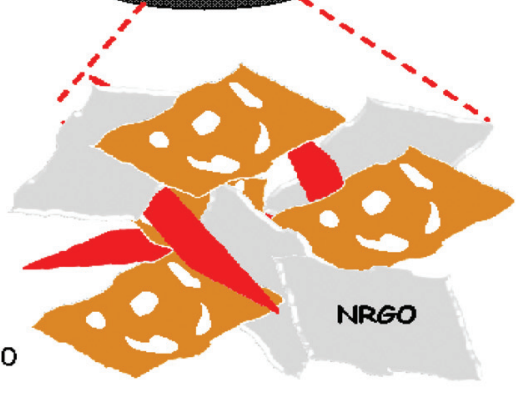

(b)

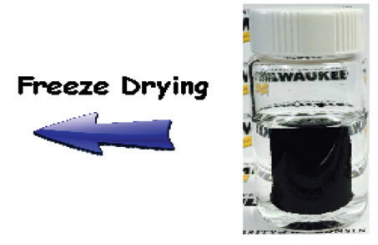

DPCN/NRGO/NiFeLDH Hydrogels

(f)

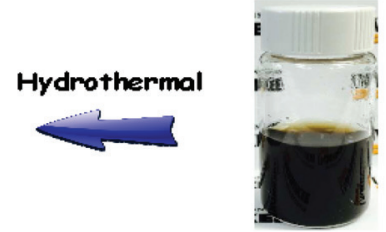

DPCN/GO/NiFe-

LDH Suspension

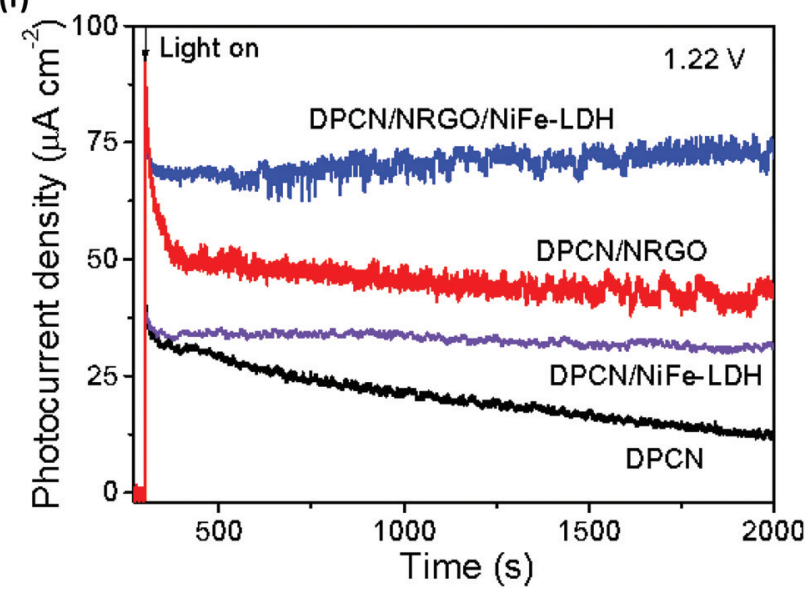

Fig. 17 (a) Schematic representation of the synthetic steps of DPCN/NRGO/NiFe-LDH. (b) Snapshots of the aerogel. (c) FESEM images of DPCN/ NRGO/NiFe-LDH. (d) EDX spectrum of DPCN/NRGO/NiFe-LDH. (e) Photocurrent vs. time plot of DPCN/NRGO/NiFe-LDH at different potentials under AM 1.5G irradiation. (f) Stability plots of DPCN, DPCN/NiFe-LDH, DPCN/NRGO, and DPCN/NRGO/NiFe-LDH under AM 1.5G exposure at 1.22 V. Adapted with permission. ${ }^{60}$ Copyright 2016, American Chemical Society.

density can be achieved only due to the synergistic effects among the shuttling of the graphene interlayer as the midlayer in promoting the separation and transfer of photoinduced exciton pairs with the co-catalytic effect of the NiFehydroxide NA as an outer layer, which enhances the stability and PEC activity of the photoanode.

Interestingly, the incorporation of $\mathrm{rGO}$ as a solid-state mediator in a $\mathrm{p}-\mathrm{n}$ heterostructure catalyst plays a great role in the efficient charge separation and the enhancement of the PEC activity of the photoanode. The role of rGO as a mediator in the NiFe-LDH/GO coupled system provides efficient charge transfer among rGO and NiFe-LDH. In this concept, Sun et al. designed a triad $\mathrm{BiVO}_{4} / \mathrm{rGO} / \mathrm{NiFe}-\mathrm{LDH}$ photoanode via a twostep facile electrodeposition method as shown in Fig. 18(a). ${ }^{134}$ The morphology of the $\mathrm{BiVO}_{4} / \mathrm{rGO} / \mathrm{NiFe}-\mathrm{LDH}$ photoanode revealed the good contact of the NiFe-LDH NS on the $\mathrm{BiVO}_{4} /$ rGO heterojunction (Fig. 18(b-d)), which facilitated the hole transfer in the PEC water oxidation. More importantly, the triad photoanode exhibited a current density of $1.13 \mathrm{~mA} \mathrm{~cm}{ }^{-2}$ at $1.23 \mathrm{~V}$ with a broad absorption in the visible zone 

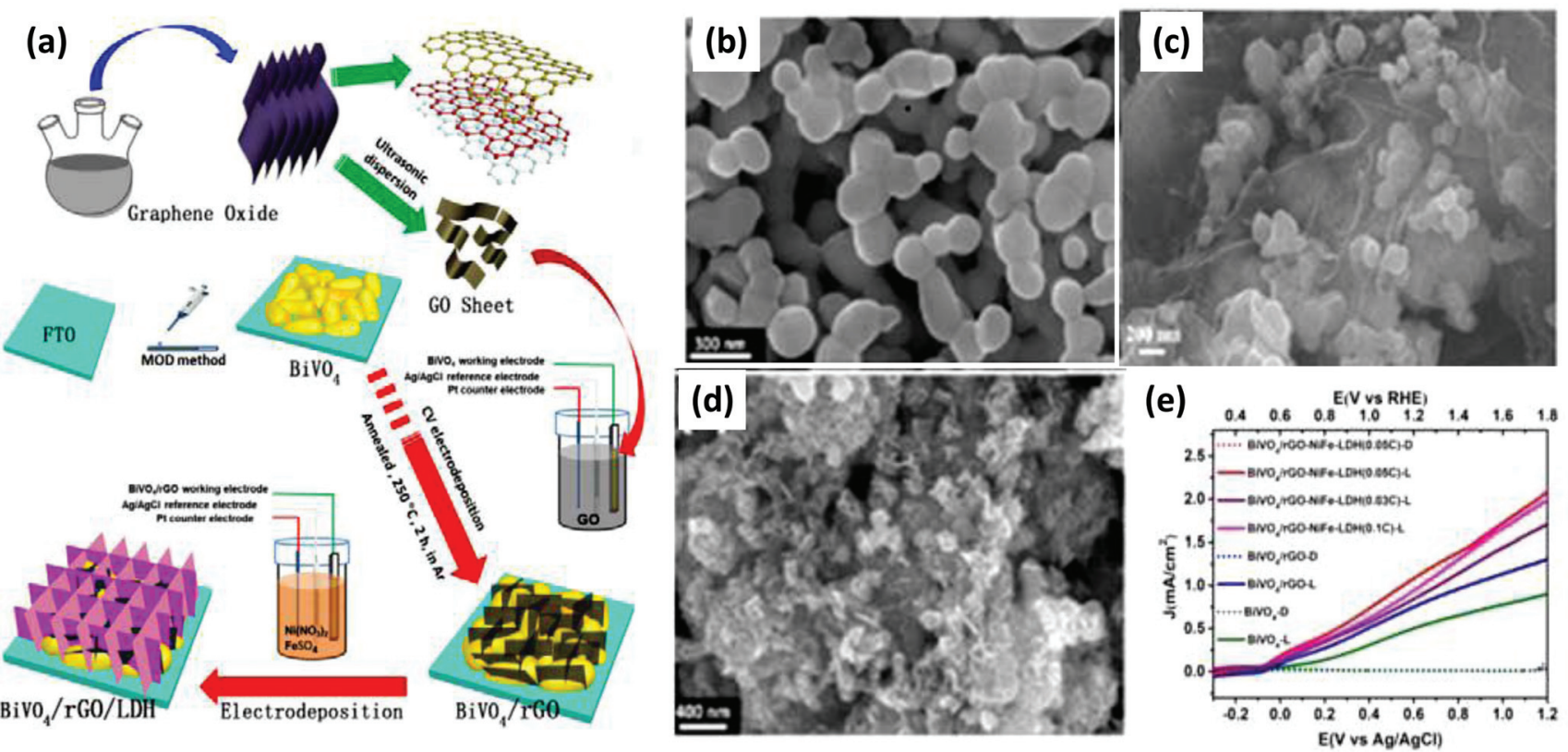

Fig. 18 (a) Schematic representation of the synthetic steps involved in $\mathrm{BiVO}_{4} / \mathrm{rGO} / \mathrm{NiFe}-\mathrm{LDH}$. SEM images of the as-synthesized (b) BiVO ${ }_{4}$ (c) $\mathrm{BiVO}_{4} / \mathrm{rGO}$ and (d) $\mathrm{BiVO}_{4} / \mathrm{rGO} / \mathrm{NiFe}-\mathrm{LDH}$. (e) Photocurrent density of $\mathrm{BiVO}_{4} / \mathrm{rGO} / \mathrm{NiFe}-\mathrm{LDH}$ with the variation in the loading quantity of LDH. ${ }^{134}$ Adapted with permission from the Royal Society of Chemistry.

(Fig. 18(e)). The supreme PEC properties of the triad photoanode were due to the following factors: (i) the creation of the $\mathrm{p}-\mathrm{n} \mathrm{rGO} / \mathrm{BiVO}_{4}$ heterojunction, (ii) the role of rGO NS as an effective mediator in the shuttling of electrons, and (iii) the role of NiFe-LDH NS as a cocatalyst in speeding up the transfer of holes during the PEC water oxidation.

Following the above major factors, Fig. 19 explains the detailed mechanism of the charge transfer in the $\mathrm{BiVO}_{4} / \mathrm{rGO} /$
NiFe-LDH triad photoanode. $\mathrm{BiVO}_{4}$, a narrow bandgap semiconductor, drastically suffers from bulk recombination. In $\mathrm{BiVO}_{4} / \mathrm{rGO}$, the fast photoinduced electrons of $\mathrm{BiVO}_{4}$ migrate towards rGO NS as a "mediator with electron shuttling" power that radiates from its delocalized electrons in the $\mathrm{sp}^{2}$ bonded carbon network, which then increases the charge carrier lifetime in the $\mathrm{BiVO}_{4} / \mathrm{rGO}$ photoanode. When NiFe-LDH as a cocatalyst couples with $\mathrm{BiVO}_{4} / \mathrm{rGO}$ then accumulates holes at the

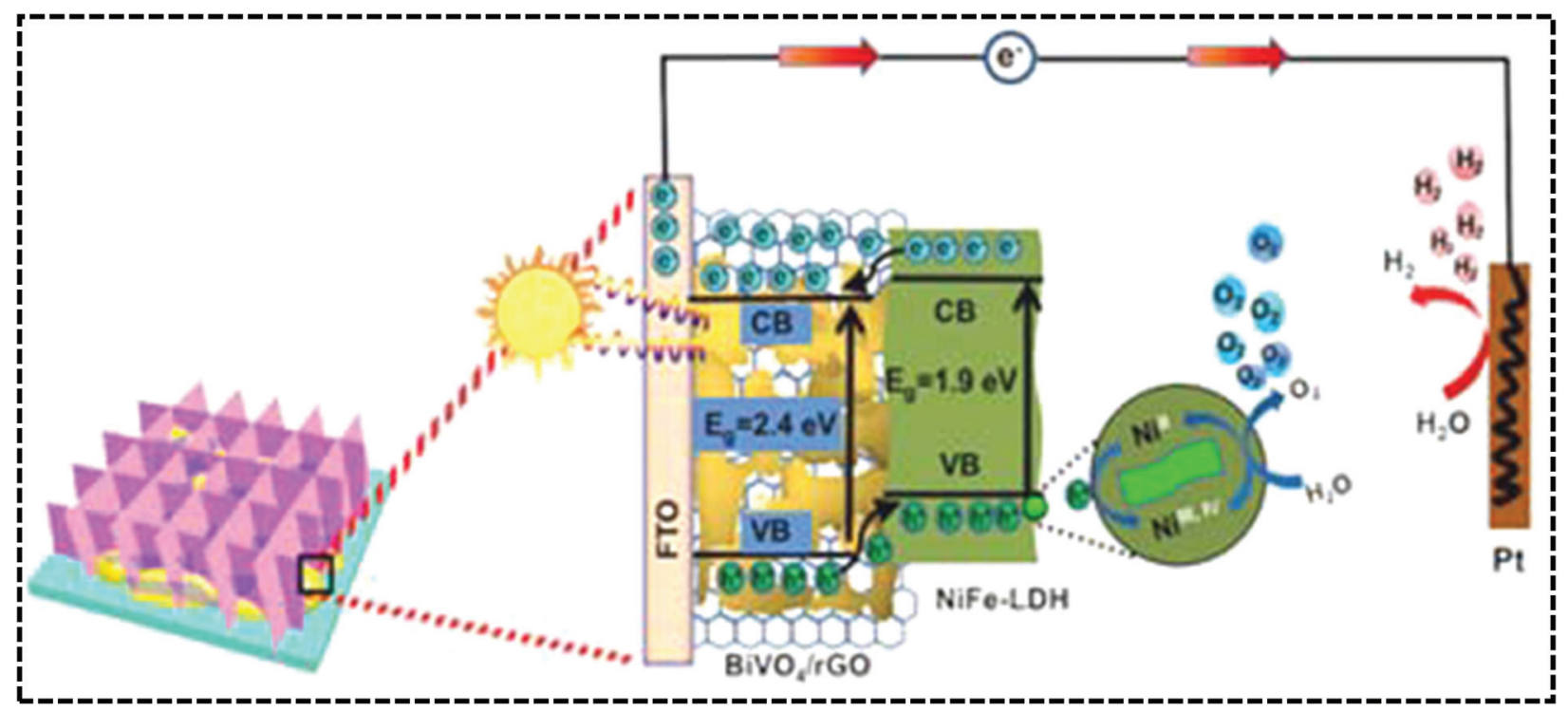

Fig. 19 Illustration of the PEC water oxidation mechanism by the $\mathrm{BiVO}_{4} / \mathrm{rGO} / \mathrm{NiFe}-\mathrm{LDH}$ ternary photoanode. ${ }^{134}$ Adapted with permission from the Royal Society of Chemistry. 
surface of NiFe-LDH, it is known as a hole collector that can facilitate water oxidation reactions. It is actually the holes of $\mathrm{BiVO}_{4}$ that participate in the redox cycle of the Ni cations of $\mathrm{NiFe}-\mathrm{LDH}$ to release oxygen. Thus, a low-cost photoanode with high PEC activity and robustness is observed in $\mathrm{BiVO}_{4} / \mathrm{rGO} /$ $\mathrm{NiFe}-\mathrm{LDH}$ as compared to the hierarchal nanopyramid $\mathrm{BiVO}_{4} /$ $\mathrm{rGO} / \mathrm{NiFe}$ hydroxide photoanode. Based on the PEC water splitting section, the different roles played by graphene in the NiFe-LDH/graphene system for enhancing the PEC activities could include an electron sink, conductive electron highway, and mediator for electron shuttling.

\section{Photocatalytic water splitting}

In 1972, Honda and Fujishima performed pioneering work on PEC water splitting using a $\mathrm{TiO}_{2}$ electrode immersed in an aqueous electrolyte solution and irradiated with UV light source. The photogenerated holes were used to oxidize $\mathrm{H}_{2} \mathrm{O}$ to form $\mathrm{O}_{2}$ and electrons were used to reduce $\mathrm{H}_{2} \mathrm{O}$ to form $\mathrm{H}_{2}$ on a Pt counter electrode via the application of an external potential by a chemical bias or power supply. ${ }^{135}$ This effort has motivated researchers regarding overall water splitting using powder particle photocatalysts for the stoichiometric evolution of $\mathrm{H}_{2}$ and $\mathrm{O}_{2}$ as perceived in 1980 by Lehn et al., ${ }^{136}$ Sato and White et al., ${ }^{137}$ and Domen et al. ${ }^{138}$ However, the UV active nature of the $\mathrm{TiO}_{2}$ photocatalyst has its limitations. Researchers have directed their interest towards water splitting using visible light-active semiconductor photocatalysts to harvest a wide portion of visible light since $4 \%$ of sunlight is UV light and the rest is visible light. Many groups have reported visible light-active photocatalysts such as $\mathrm{Bi}_{2} \mathrm{O}_{3}, \mathrm{WO}_{3}$, $\mathrm{BiVO}_{4}, \mathrm{CdS}$, etc. Although these materials have established promising performances through intensive studies, there are some limitations such as short lifetimes, toxicity, instability due to photocorrosion or self-oxidation, and low efficiency towards visible light absorption, which restrict their practical applications. 5,139,140 Regarding photocatalytic performance, efficiency and stability, the development of more advanced, robust and extremely competent semiconductor photocatalysts for photocatalytic water splitting is still a challenge and more efforts are needed for the advancement of robust photocatalysts. ${ }^{141-143}$ Heterogeneous photocatalytic water splitting is a green technology that introduces dispersed powdered photocatalysts and is more beneficial than the PEC technique because of its facile approach, sustainability, cost-effectiveness and external potential. ${ }^{33-37,44}$ Generally, semiconductor photocatalysts used in photocatalytic water splitting depend on several necessities concerning the bandgap energy and electronic properties as follows. (i) The photoactive semiconductor must possess a band structure, inside which the differences in the position of the $\mathrm{CB}$ and VB create a bandgap and should harvest the visible light of the solar spectrum; (ii) it should be capable of separating photoinduced $\mathrm{e}^{-}$from reactive $\mathrm{h}^{+}$; (iii) a minimum amount of energy should be dissipated during charge transportation and recombination by photoinduced charge carriers; (iv) there should be chemical stability in aqueous media; (v) it should be kinetically active for electron transportation from the surface of a photocatalyst to the water interface, and (vi) possess appropriate band edge potentials for overall water splitting e.g., the $\mathrm{CB}$ of a photocatalyst should be situated at a higher negative potential than the $\mathrm{H}_{2} \mathrm{O}$ reduction potential $\left(\mathrm{H}^{+} / \mathrm{H}_{2}=0 \mathrm{~V}\right.$ vs. NHE, $\left.\mathrm{pH}=0\right)$. Similarly, the VB of a photocatalyst must surpass the $\mathrm{H}_{2} \mathrm{O}$ oxidation potential to facilitate the oxidation reaction $(+1.23 \mathrm{~V} v s$. NHE, $\mathrm{pH}$ 0). Consequently, a theoretical semiconductor bandgap energy of $\Delta G=+237 \mathrm{~kJ} \mathrm{~mol}^{-1}$ or $1.23 \mathrm{eV}$ is required to force the overall water-splitting (eqn (1.6)-(1.8)). This amount of energy is comparable to a photon energy of $\lambda=1010 \mathrm{~nm}$, with bandgap $(\mathrm{eV})=1240 / \lambda(\mathrm{nm})$. Hence, theoretically, ca. $70 \%$ of each solar photon is accessible for water splitting. ${ }^{136}$ All these steps are critical for photocatalytic water splitting reactions. Thus, a robust photocatalyst must possess the appropriate electronic band structure, surface characteristics and bulk properties to contribute to water splitting. The reduction and oxidation reactions of water splitting are as shown in the following equations:

$$
\begin{gathered}
\text { Oxidation } \mathrm{H}_{2} \mathrm{O}+2 \mathrm{~h}^{+} \rightarrow 2 \mathrm{H}^{+}+\frac{1}{2} \mathrm{O}_{2} \\
\text { Reduction } \mathrm{H}^{+}+2 \mathrm{e}^{-} \rightarrow \mathrm{H}_{2} \\
\text { Overall water splitting } \mathrm{H}_{2} \mathrm{O} \rightarrow \mathrm{H}_{2}+\frac{1}{2} \mathrm{O}_{2}
\end{gathered}
$$

Half-reactions of water splitting proceed by using sacrificial agents and are mainly suitable for deciding whether or not a specified semiconductor satisfies the thermodynamic and kinetic requirements for the liberation of $\mathrm{H}_{2}$ and $\mathrm{O}_{2}$. Reducing agents such as alcohol (methanol, ethanol), triethylamine (TEA), triethyloxysulphides (TEOS), sulphites and ethylenediaminetetraacetate (EDTA) have been used as sacrificial agents for water reduction, and oxidizing reagents such as persulphate, $\mathrm{AgNO}_{3}, \mathrm{Fe}^{3+}$ have been used as sacrificial agents to facilitate water oxidation. However, overall water splitting by using only pure $\mathrm{H}_{2} \mathrm{O}$ in the absence of sacrificial reagents fails to give good results. Alternatively, photoinduced electrons at the $\mathrm{CB}$ edge of a semiconductor photocatalyst are attracted by oxidizing agents (electron acceptors or electron scavengers) such as $\mathrm{AgNO}_{3}$, and irreversibly reduce the oxidizing agent as an alternative to $\mathrm{H}_{2} \mathrm{O}$. They augment holes in the VB of a photocatalyst and enhance the liberation of $\mathrm{O}_{2}$ gas. The following equations are involved in photocatalytic $\mathrm{H}_{2}$ production using methanol as a sacrificial reagent. The product formaldehyde (eqn (1.15)) is then oxidized to methanoic acid ( $\mathrm{HCOOH})$ and then to $\mathrm{CO}_{2}$ and $\mathrm{H}_{2}$ as described by eqn (1.16) and (1.17).

$$
\begin{gathered}
\text { Semiconductor Photocatalyst } \stackrel{h \nu}{\longrightarrow} \mathrm{e}^{-}+\mathrm{h}^{+} \\
\qquad \begin{array}{c}
\mathrm{h}^{+}+\mathrm{H}_{2} \mathrm{O} \rightarrow \cdot \mathrm{OH}+\mathrm{H}^{+} \\
2 \mathrm{H}^{+}+2 \mathrm{e}^{-} \rightarrow \mathrm{H}_{2}
\end{array}
\end{gathered}
$$




$$
\begin{gathered}
\mathrm{CH}_{3} \mathrm{OH}+{ }^{\cdot} \mathrm{OH} \rightarrow{ }^{\cdot} \mathrm{CH}_{2} \mathrm{OH}+\mathrm{H}_{2} \mathrm{O} \\
\mathrm{CH}_{2} \mathrm{OH} \rightarrow \mathrm{HCHO}+\mathrm{H}^{+}+\mathrm{e}^{-} \\
2 \mathrm{H}_{2} \mathrm{O}+2 \mathrm{e}^{-} \rightarrow \mathrm{H}_{2}+2 \mathrm{OH}^{-} \\
\mathrm{CH}_{3} \mathrm{OH} \stackrel{h \nu, \text { Catalyst }}{\longrightarrow} \mathrm{HCHO}+\mathrm{H}_{2} \\
\mathrm{HCHO}+\mathrm{H}_{2} \mathrm{O} \stackrel{h \nu, \text { Catalyst }}{\longrightarrow} \mathrm{HCOOH}+\mathrm{H}_{2} \\
\mathrm{HCOOH} \stackrel{h \nu, \text { Catalyst }}{\longrightarrow} \mathrm{CO}_{2}+\mathrm{H}_{2}
\end{gathered}
$$

Photoinduced excitonic charge recombination is a major obstacle for photocatalytic water splitting reactions. The concept of heterostructure formation is an effective approach to inhibit the charge recombination and the assembly of the $\mathrm{NiFe}-\mathrm{LDH} /$ graphene-coupled system is a topic of interest. NiFe$\mathrm{LDH}$ with its strong optical response and unique structure has become a hot material for photocatalytic applications. The metal centers in NiFe-LDH are connected by 'oxo-bridged' linkages, ${ }^{144-146}$ which serve as potential sites for charge separation and trigger the optical response of these materials. Also, the presence of the hydroxyl group oxidizes holes in a homogeneous reaction. ${ }^{62}$ Alternatively, graphene is recognized as a substrate material, carrier, mediator and a cocatalyst for photocatalytic water splitting reactions owing to its higher specific surface area and easier carrier charge mobility. ${ }^{33}$ We have also reported different NiFe-LDH-based heterostructure materials for various light-driven photocatalytic reactions such as in g- $\mathrm{C}_{3} \mathrm{~N}_{4} / \mathrm{NiFe}-\mathrm{LDH},{ }^{27} \mathrm{Ag} @ \mathrm{Ag}_{3} \mathrm{PO}_{4} / \mathrm{g}-\mathrm{C}_{3} \mathrm{~N}_{4} / \mathrm{NiFe}-\mathrm{LDH},{ }^{62} \mathrm{NiFe}-$ $\mathrm{LDH} / \mathrm{N}-\mathrm{rGO} / \mathrm{g}-\mathrm{C}_{3} \mathrm{~N}_{4},{ }^{33}$ and $\mathrm{MoS}_{2} / \mathrm{NiFe}-\mathrm{LDH}{ }^{34}$ For the first time, in our work, g- $\mathrm{C}_{3} \mathrm{~N}_{4} / \mathrm{NiFe}-\mathrm{LDH}$ has provided deep insight into the heterojunction/nanocomposite effect of $\mathrm{g}-\mathrm{C}_{3} \mathrm{~N}_{4} / \mathrm{LDH}$ for targeted photocatalytic $\mathrm{H}_{2}$ and $\mathrm{O}_{2}$ production. In this regard, the coupling of $\mathrm{g}-\mathrm{C}_{3} \mathrm{~N}_{4}$ with $\mathrm{LDH}$ semiconductors could be a remarkable approach to improving the photocatalytic performance owing to the 2D-2D layered structure, which is of great advantage for utilizing full electronic coupling for effective charge separation. The morphology and chemical composition of LDHs can be delicately tailored to achieve the targeted charge separation efficiency as well as electrical conductivity. ${ }^{27}$

Researchers and scientists are quite interested in developing robust catalysts with strong redox and effective charge separation ability that could strike the most productive results in water splitting. As discussed in previous sections, heterostructure formation can only meet these entire criteria. NiFe$\mathrm{LDH} /$ graphene in combination with other semiconductors can form heterojunctions in which graphene can act as a mediator for the spatial separation of exciton pairs while preserving higher redox potentials. However, there are no reports of NiFe$\mathrm{LDH} /$ graphene for photocatalytic water splitting except for our breakthrough work, i.e., NiFe-LDH/N-rGO/g- $\mathrm{C}_{3} \mathrm{~N}_{4} \cdot{ }^{33}$ In this work, the heterostructured NiFe-LDH/N-rGO/g- $\mathrm{C}_{3} \mathrm{~N}_{4}$ was constructed by a facile calcination-electrostatic self-assemblyhydrothermal method as shown in Fig. 7. N-rGO exhibits immense advancement in electrical conductivity linked with the incorporation of the nitrogen heteroatom and also assumes the qualities of graphene such as the outstanding physicochemical properties and large theoretical surface area. In NiFe-LDH/N-rGO/g- $\mathrm{C}_{3} \mathrm{~N}_{4}, \mathrm{~N}-\mathrm{rGO}$ acts as a solid-state electronic mediator, integrated between NiFe-LDH and $\mathrm{g}-\mathrm{C}_{3} \mathrm{~N}_{4}$ for superior PC reactions. This catalyst displayed outstanding PC $\mathrm{H}_{2}$ and $\mathrm{O}_{2}$ evolution rates of $2508 \mu \mathrm{mol} \mathrm{g} \mathrm{g}^{-1} 2 \mathrm{~h}^{-1}$ and $1280 \mu \mathrm{mol} \mathrm{g}{ }^{-1} 2 \mathrm{~h}^{-1}$, respectively (Fig. 20(a and b)), and also displayed exceptional catalytic performances in degrading 95\% of Rhodamine B (RhB) dye and 72\% of phenol (Fig. 20(c-e)). The role of graphene in the ternary NiFe-LDH/N-rGO/g- $\mathrm{C}_{3} \mathrm{~N}_{4}$ heterostructure for photocatalytic water splitting can be clarified by analyzing the mechanism of charge separation. The coupling of $\mathrm{N}$-doped rGO with catalytic active sites on NiFeLDH triggered the dynamics of the charge migration phenomenon at the interfacial area of $\mathrm{LDH} / \mathrm{g}-\mathrm{C}_{3} \mathrm{~N}_{4}$. In the mechanistic approach, NiFe-LDH NS and $\mathrm{g}-\mathrm{C}_{3} \mathrm{~N}_{4} \mathrm{NS}$ form strong electrostatic bonds with the N-rGO surface, where the $\mathrm{CB}$ electrons of $\mathrm{LDH}$ recombine with the $\mathrm{VB}$ holes of $\mathrm{g}-\mathrm{C}_{3} \mathrm{~N}_{4}$. The $\mathrm{N}$-rGO mediator assists in decreasing the charge transportation time, lengthens the lifetime of charge carriers, and endorses the separation of photoinduced carriers charge pairs. In this way, $\mathrm{N}$-rGO near the flat band potential $\left(E_{\mathrm{fb}}\right)$ of NiFe-LDH and $\mathrm{g}-\mathrm{C}_{3} \mathrm{~N}_{4}$ can increase the kinetics of water reduction and oxidation potential of the ternary heterostructure. Such attempts indicate the importance of rGO in the charge transport dynamics next to the interfacial area of $\mathrm{LDH} / \mathrm{g}-\mathrm{C}_{3} \mathrm{~N}_{4}$, as well as the visible-light-harvesting ability of the heterostructure. As realized from the PC water splitting study, graphene mostly plays the role of mediator in NiFe-LDH/graphene-based systems for superior PC performance (Table 1).

\section{Conclusions and outlook}

We have summarized an updated strategy for the design of the high-performance NiFe-LDH/graphene nanocomposites for promising energy conversion applications. NiFe-LDH/graphene nanocomposites possess many advantages as follows: (1) they incorporate the advantages and diminish the shortcomings of the solitary materials. (2) The low electrical conductivity of $\mathrm{NiFe}-\mathrm{LDH}$ is corrected by the support of the conductive graphene matrix. (3) Intercalated graphene acts as a protective layer, effectively restricting the self-aggregation of NiFe-LDH NS and thereby improving the stability. (4) They expose more catalytically active sites due to the good contact between Ni/Fe atoms and doped graphene. (5) The spatially confined growth of nanosized NiFe-LDH in the mesopores of 3D-graphene opens up many coordinate sites for the catalytic process. In the synthesis strategy, the recent advancement in new synthesis protocols for the NiFe-LDH/graphene hybrid with precise structure, morphology and functions are summarized. The in situ growth strategy summarizes the controlled hybridization of the active phases of NiFe-LDH into the conductive graphene substrate, mostly controlling the composition and morphology under different reaction parameters and the effect on 
(a)

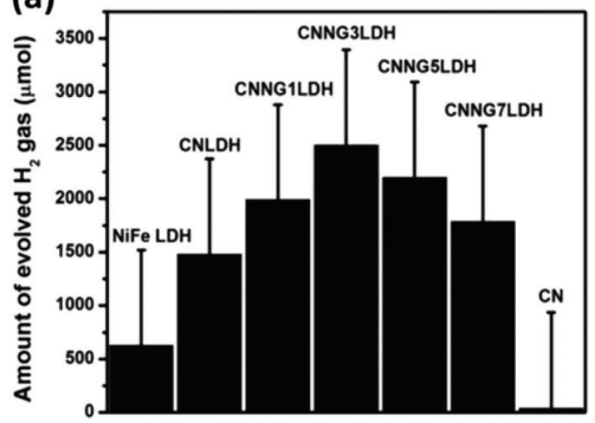

(d) 2.5

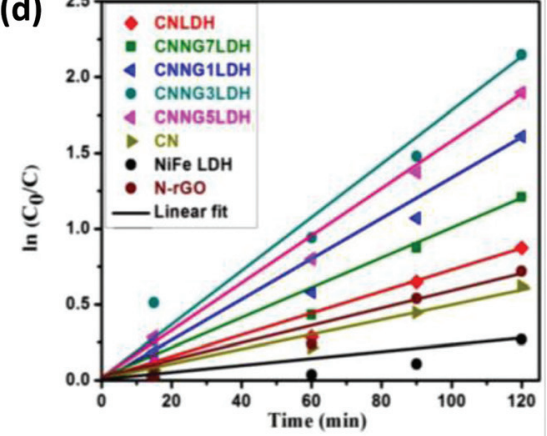

(b)

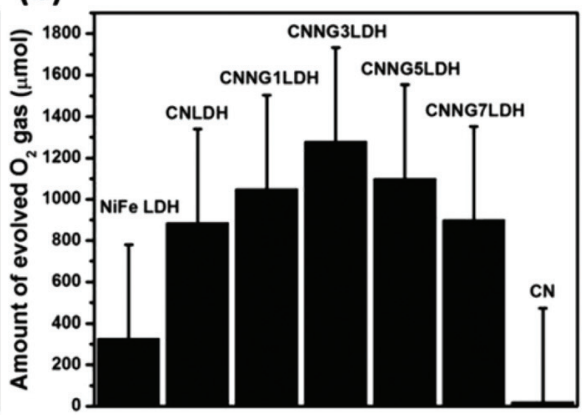

(e)

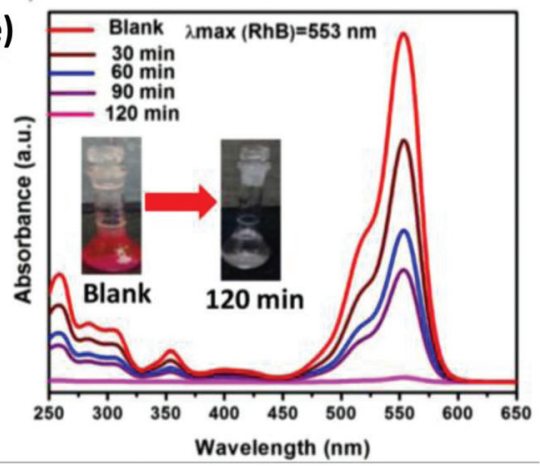

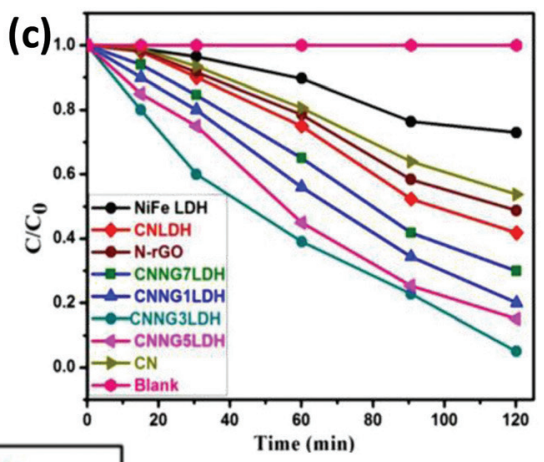

Fig. 20 (a) Plots of the quantity of $\mathrm{H}_{2}$ evolved ( $\mu \mathrm{mol} \mathrm{g} \mathrm{g}^{-1}$ ). (b) Plots of the quantity of $\mathrm{O}_{2}$ released $\left(\mu \mathrm{mol} \mathrm{g}^{-1}\right)$ on NiFe LDH (LDH), g- $\mathrm{C}_{3} \mathrm{~N}_{4}(\mathrm{CN})$, CNLDH (CNNGx, $x=1,3,5$ and 7 wt\% of GO w.r.t. the quantities of NiFe-LDH $(1 \mathrm{~g})$ ) and CNNGxLDH. Variation of N-rGO on CNLDH, i.e., $x=1,3,5$ and 7 wt\% of GO against NiFe LDH in CNNGx. Adapted with permission from ref. 33, Copyright 2019 Nature.

the water splitting reactions. The in situ growth also forms strong bonding interactions between NiFe-LDH and graphene, providing the materials with the desired morphologies. Alternatively, the heteroassembly of NiFe-LDH/graphene summarizes the favorable modes of interaction that form a stable and active structure for efficient water splitting; for example, face-to-face contact between NiFe-LDH NS and GO NS through the layer-by-layer approach allows strong interfacial interactions that expose more catalytic active sites for water splitting. It is worth noting that a synergistic effect is realized in the resultant heterostructure and the performances of the NiFe-LDH/graphene nanohybrid, which are fully dependent on the dimensions of graphene materials, the pre-treatment of $\mathrm{NiFe}-\mathrm{LDH}$ and the reaction conditions. Firstly, the NiFe-LDH/ 2D-graphene composites possess larger specific surface areas, superior electrical conductivity and dimensional orientation, which expose a huge population of active sites of metal centers and boost the charge particles for superior water splitting in EC/PEC/PC. Secondly, the NiFe-LDH/3D-graphene structure exhibits a more axial orientation than the 2D-graphene based nanohybrids and offers a potential substrate for the dispersion of a huge population of catalytically active sites. It also has properties like hierarchical porosity, entangled structure, multidirectional electron mobility and enhanced conductivity, which are responsible for the superior EC/PEC performances.

For an effective energy conversion process, many systematic improvements are needed regarding the fabrication and synthesis of NiFe-LDH/graphene nanocomposites. Accordingly, the research aspects and future prospectives of NiFe-LDH/graphene nanocomposites are as follows:

(i) It is important to directly form 3D freestanding electrodes or grow spatially confined $\mathrm{LDH}$ on a conductive 3D graphene matrix due to the creation of more defect sites and enhanced properties that are different from their precursor material, which will facilitate superior electrocatalytic activities with long-term stability; however, there is a long way to go to meet the requirements of the industry. Hence, in-depth studies on the design of free-standing electrodes and coupled catalytic systems using $\mathrm{NiFe-LDH/graphene} \mathrm{are} \mathrm{required} \mathrm{for}$ potential applications in electrocatalytic devices.

(ii) The advantages of $\mathrm{LDH}$ materials can be partly due to their derivatives such as metal oxides, oxyhydroxides, hydroxides, phosphides, sulfides, bimetal nitrides, and selenides. These LDH derivatives exhibit outstanding EC activities owing to their modulated local electronic environments, enhanced electrical conductivity and improved corrosion resistance properties. However, less work has been conducted on the morphological and topological control in LDH derived mixed metal oxides and nitrides towards water splitting. Moreover, $\mathrm{NiFe}-\mathrm{LDH}$ and their derivatives are complicated owing to the overlapping of the $\mathrm{Ni}^{2+} / \mathrm{Ni}^{3+}$ redox cycle, the OER current density, the onset potential and the Tafel slope. Therefore, there is plenty of room for the optimization of the electronic structure of NiFe-LDH derivatives by computational methods and morphological control by a simple experimental set-up for advanced catalytic properties. 


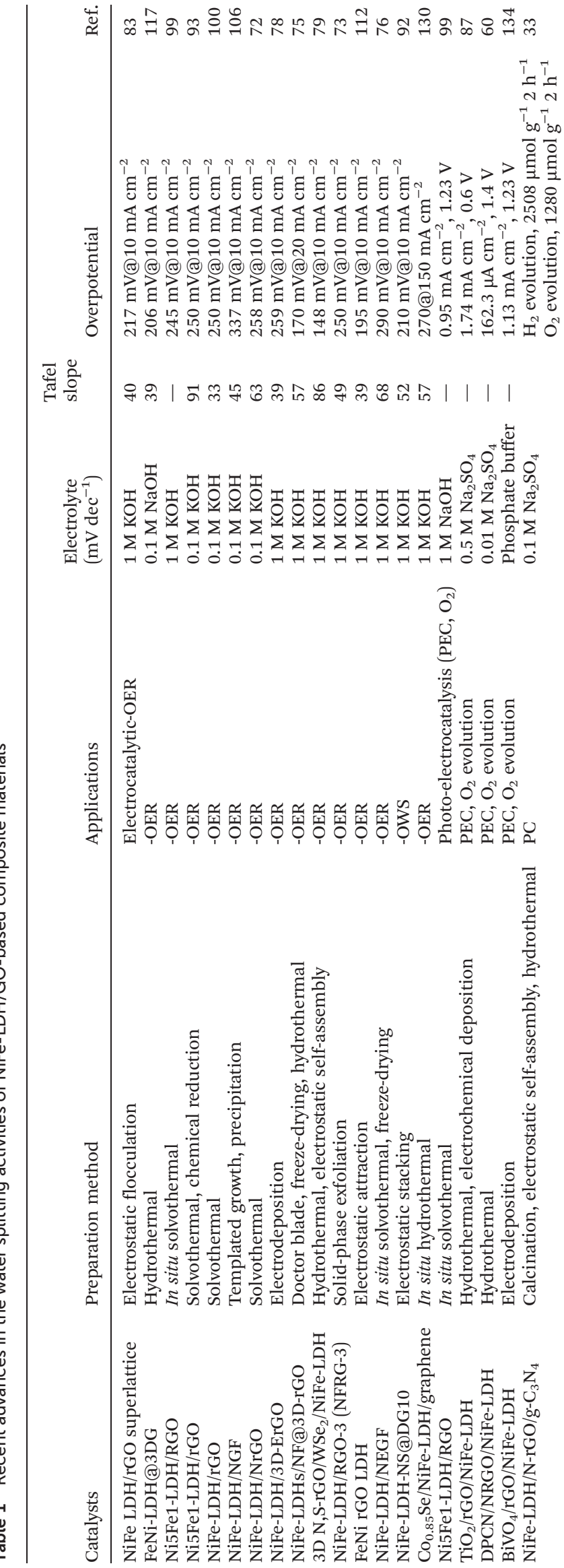

뜐 은 (iii) Understanding the structure and activity in terms of interfacial coupling interactions or interface modulation between NiFe-LDHs and graphene for the formation of a superlattice structure can provide information on NiFe-LDH/ graphene nanocomposites, which is advantageous for catalytic performance and stability. Therefore, in-depth comparisons with other superlattice structures and insight into the catalytic mechanism of NiFe-LDH/graphene are highly recommended. Further advances of NiFe-LDH/graphene toward promising energy storage are needed, and more exploration of NiFe-LDH/ graphene towards photocatalytic and photoelectrochemical water splitting is desired.

(iv) Identification of the active sites in NiFe-LDH/graphene under operando conditions and the mechanism of catalyst action can provide a depth understanding of the structure and activity correlation of the associated catalysts and the design of novel materials with enhanced activities. The in situ characterization of the local electronic structure by using fast-growing powerful instrumental techniques under operando conditions provides fundamental principles for the working catalysts. More specifically, the identification of the most active $\gamma$-NiOOH phase from the anhydrous $\beta$-NiOOH phase in NiFeLDH during the OER has long been a debate. Although some studies have made great efforts by combining operando experiments and DFT computational studies to justify the elevated OER performance of the NiFe-LDHs on the atomic level, it is still unclear. Therefore, the systematic identification of the catalytic route and operational active phases of NiFe-LDHs in the NiFe-LDH/graphene model with variations in the atomic ratio, composition and physical structure by using sophisticated online, computational and operando conditions are strongly expected.

\section{Conflicts of interest}

There are no conflicts to declare.

\section{Acknowledgements}

This research work is graciously financed by the management of Siksha 'O' Anusandhan Deemed to be University.

\section{References}

1 C. J. Quarton, O. Tlili, L. Welder, C. Mansilla, H. Blanco, H. Heinrichs, J. Leaver, N. J. Samsatli, P. Lucchese, M. Robinius and S. Samsatli, The curious case of the conflicting roles of hydrogen in global energy scenarios, Sustainable Energy Fuels, 2020, 4, 80-95.

$2 \mathrm{H}$. Ibrahim, A. Ilinca and J. Perron, Energy storage systems-characteristics and comparisons, Renewable Sustainable Energy Rev., 2008, 12, 1221-1250.

3 A. B. Gallo, J. R. Simoes-Moreira, H. K. M. Costa, M. M. Santos and E. Moutinho dos Santos, Energy storage 
in the energy transition context: A technology review, Renewable Sustainable Energy Rev., 2016, 65, 800-822.

4 N. S. Lewis, Toward cost-effective solar energy use, Science, 2007, 315, 798-801.

5 A. Kudo and Y. Miseki, Heterogeneous photocatalyst materials for water splitting, Chem. Soc. Rev., 2009, 38, 253-278.

6 M. Shao, R. Zhang, Z. Li, M. Wei, D. G. Evans and $\mathrm{X}$. Duan, Layered double hydroxides toward electrochemical energy storage and conversion: design, synthesis and applications, Chem. Commun., 2015, 51, 1588015893.

7 C. Chen, L. Tao, S. Du, W. Chen, Y. Wang, Y. Zou and S. Wang, Advanced exfoliation strategies for layered double hydroxides and applications in energy conversion and storage, Adv. Funct. Mater., 2020, 30, 19098321909849.

8 Y. Zhao, X. Jia, G. I. N. Waterhouse, L. Z. Wu, C. H. Tung, D. O'Hare and T. Zhang, Layered double hydroxide nanostructured photocatalysts for renewable energy production, Adv. Energy Mater., 2016, 6, 1501974-1501994.

9 Y. Xu, M. Kraft and R. Xu, Metal-free carbonaceous electrocatalysts and photocatalysts for water splitting, Chem. Soc. Rev., 2016, 45, 3039-3052.

10 T. Li, T. Kasahara, J. He, K. E. Dettelbach, G. M. Sammis and C. P. Berlinguette, Photoelectrochemical oxidation of organic substrates in organic media, Nat. Commun., 2017, 8, 390-394.

11 Y. X. Chen, A. Lavacchi, H. A. Miller, M. Bevilacqua, J. Filippi, M. Innocenti, A. Marchionni, W. Oberhauser, L. Wang and F. Vizza, Nanotechnology makes biomass electrolysis more energy efficient than water electrolysis, Nat. Commun., 2014, 5, 4036-4035.

12 M. Carmo, D. L. Fritz, J. Mergel and D. Stolten, A comprehensive review on PEM water electrolysis, Int. J. Hydrogen Energy, 2013, 38, 4901-4934.

13 L. Lv, Z. Yang, K. Chen, C. Wang and Y. Xiong, 2D layered double hydroxides for oxygen evolution reaction: from fundamental design to application, Adv. Energy Mater., 2019, 9, 1803358-1803386.

14 M. Z. Iqbal and S. Siddique, Recent progress in efficiency of hydrogen evolution process based photoelectrochemical cell, Int. J. Hydrogen Energy, 2018, 43, 21502-21523.

$15 \mathrm{R} . \mathrm{Li}$, Latest progress in hydrogen production from solar water splitting via photocatalysis, photoelectrochemical, and photovoltaic-photoelectrochemical solutions, Chin. J. Catal., 2017, 38, 5-12.

16 R. Abe, Recent progress on photocatalytic and photoelectrochemical water splitting under visible light irradiation, J. Photochem. Photobiol., C, 2010, 11, 179-209.

17 K. Maeda and K. Domen, Photocatalytic water splitting: recent progress and future challenges, J. Phys. Chem. Lett., 2010, 1, 2655-2661.

18 X. Chen, S. Shen, L. Guo and S. S. Mao, Semiconductorbased photocatalytic hydrogen generation, Chem. Rev., 2010, 110, 6503-6570.
19 H. Zhao and Z. Y. Yuan, Transition metal-phosphorusbased materials for electrocatalytic energy conversion reactions, Catal. Sci. Technol., 2017, 7, 330-347.

20 A. K. Tareen, G. S. Priyanga, K. Khan, E. Pervaiz, T. Thomas and M. Yang, Nickel-based transition metal nitride electrocatalysts for the oxygen evolution reaction, ChemSusChem, 2019, 12, 3941-3954.

21 G. Fu and J. M. Lee, Ternary metal sulfides for electrocatalytic energy conversion, J. Mater. Chem. A, 2019, 7, 9386-9405.

22 H. Wan, X. Liu, H. Wang, R. Ma and T. Sasaki, Recent advances in developing high-performance nanostructured electrocatalysts based on 3d transition metal elements, Nanoscale Horiz., 2019, 4, 789-808.

23 V. Rives and M. A. Ulibarri, Layered double hydroxides (LDH) intercalated with metal coordination compounds and oxometalates, Coord. Chem. Rev., 1999, 181, 61-120.

24 E. Manasse and A. S. Toscana, The group 13 metals Aluminum, Gallium, Indium and Thallium chemical pattern and peculiarities, Sc. Nat. Proc. Verb., 1915, 24, 9295.

25 R. Allmann, The crystal structure of pyroaurite, Acta Crystallogr., Sect. B: Struct. Crystallogr. Cryst. Chem., 1968, 24, 972-977.

26 H. F. W. Taylor, Crystal structures of some double hydroxide Minerals, Mineralogical Magazine, Mineral. Mag., 1969, 37, 338-342.

27 S. Nayak, L. Mohapatra and K. M. Parida, Visible lightdriven novel g- $\mathrm{C}_{3} \mathrm{~N}_{4} / \mathrm{NiFe}-\mathrm{LDH}$ composite photocatalyst with enhanced photocatalytic activity towards water oxidation and reduction reaction, J. Mater. Chem. A, 2015, 3, 18622-18635.

28 S. Nayak and K. M. Parida, Nanostructured $\mathrm{CeO}_{2} / \mathrm{MgAl}-$ LDH composite for visible light induced water reduction reaction, Int. J. Hydrogen Energy, 2016, 41, 21166-21180.

29 Y. Zhao, X. Jia, G. I. N. Waterhouse, L. Z. Wu, C. H. Tung, D. O'Hare and T. Zhang, Layered double hydroxide nanostructured photocatalysts for renewable energy production, Adv. Energy Mater., 2016, 6, 1501974-1501994.

30 S. Das, S. K. Dash and K. M. Parida, Kinetics, isotherm, and thermodynamic study for ultrafast adsorption of Azo dye by an efficient sorbent: ternary $\mathrm{Mg} /(\mathrm{Al}+\mathrm{Fe})$ Layered double hydroxides, ACS Omega, 2018, 3, 25322545.

31 R. Chen, S. F. Hung, D. Zhou, J. Gao, C. Yang, H. Tao, H. B. Yang, L. Zhang, L. Zhang, Q. Xiong, H. M. Chen and B. Liu, Layered structure causes bulk NiFe layered double hydroxide unstable in alkaline oxygen evolution reaction, Adv. Mater., 2019, 31, 1903909-1903915.

32 M. Gong, Y. Li, H. Wang, Y. Liang, J. Z. Wu, J. Zhou, J. Wang, T. Regier, F. Wei and H. Dai, An advanced Ni-Fe layered double hydroxide electrocatalyst for water oxidation, J. Am. Chem. Soc., 2013, 135, 8452-8455.

33 S. Nayak and K. M. Parida, Deciphering Z-scheme charge transfer dynamics in heterostructure NiFe-LDH/N-rGO/ $\mathrm{g}^{-} \mathrm{C}_{3} \mathrm{~N}_{4}$ nanocomposite for photocatalytic pollutant 
removal and water splitting reactions, Sci. Rep., 2019, 9, 2458-2481.

34 S. Nayak, G. Swain and K. Parida, Enhanced photocatalytic activities of $\mathrm{RhB}$ degradation and $\mathrm{H}_{2}$ evolution from in situ formation of the electrostatic heterostructure $\mathrm{MoS}_{2} / \mathrm{NiFe} \mathrm{LDH}$ nanocomposite through the Z-scheme mechanism via $\mathrm{p}-\mathrm{n}$ heterojunctions, ACS Appl. Mater. Interfaces, 2019, 11, 20923-20942.

35 S. Nayak, A. C. Pradhan and K. M. Parida, Topotactic transformation of solvated $\mathrm{MgCr}-\mathrm{LDH}$ nanosheets to highly efficient porous $\mathrm{MgO} / \mathrm{MgCr}_{2} \mathrm{O}_{4}$ nanocomposite for photocatalytic $\mathrm{H}_{2}$ evolution, Inorg. Chem., 2018, 57, 86468661.

36 D. P. Sahoo, S. Patnaik, D. Rath and K. M. Parida, Synergistic effects of plasmon induced $\mathrm{Ag}$ @ $\mathrm{Ag}_{3} \mathrm{VO}_{4} / \mathrm{ZnCr}$ LDH ternary heterostructures towards visible light responsive $\mathrm{O}_{2}$ evolution and phenol oxidation reactions, Inorg. Chem. Front., 2018, 5, 879-896.

37 D. P. Sahoo, S. Nayak, K. H. Reddy, S. Martha and K. Parida, Fabrication of a $\mathrm{Co}(\mathrm{OH})_{2} / \mathrm{ZnCr} \mathrm{LDH}$ "p-n" heterojunction photocatalyst with enhanced separation of charge carriers for efficient visible-light-driven $\mathrm{H}_{2}$ and $\mathrm{O}_{2}$ evolution, Inorg. Chem., 2018, 57, 3840-3854.

38 S. Acharya and K. Parida, A visible light-driven $\mathrm{Zn} / \mathrm{Cr}-$ $\mathrm{LaFeO}_{3}$ nanocomposite with enhanced photocatalytic activity towards $\mathrm{H}_{2}$ production and $\mathrm{RhB}$ degradation, ChemistrySelect, 2017, 2, 10239-10248.

39 F. Dionigi, Z. Zeng, I. Sinev, T. Merzdorf, S. Deshpande, M. B. Lopez, S. Kunze, I. Zegkinoglou, H. Sarodnik, D. Fan, A. Bergmann, J. Drnec, J. F. de Araujo, M. Gliech, D. Teschner, J. Zhu, W. X. Li, J. Greeley, B. R. Cuenya and P. Strasser, In situ structure and catalytic mechanism of $\mathrm{NiFe}$ and CoFe layered double hydroxides during oxygen evolution, Nat. Commun., 2020, 11, 2522-2531.

40 S. Das, S. Patnaik and K. M. Parida, Fabrication of a Auloaded $\mathrm{CaFe}_{2} \mathrm{O}_{4} / \mathrm{CoAl}$ LDH p-n junction based architecture with stoichiometric $\mathrm{H}_{2} \& \mathrm{O}_{2}$ generation and $\mathrm{Cr}$ (vi) reduction under visible light, Inorg. Chem. Front., 2019, 6, 94-109.

41 S. Jiang, Y. Liu, W. Xie and M. Shao, Electrosynthesis of hierarchical NiLa-layered double hydroxide electrode for efficient oxygen evolution reaction, J. Energy Chem., 2019, 33, 125-129.

42 J. Chen, X. Wang, J. Wang and P. S. Lee, Sulfidation of NiMn-layered double hydroxides/graphene oxide composites toward supercapacitor electrodes with enhanced performance, Adv. Energy Mater., 2016, 6, 1501745-1501752.

43 Y. Zhao, J. He, M. Dai, D. Zhao, X. Wu and B. Liu, Emerging CoMn-LDH@ $\mathrm{MnO}_{2}$ electrode materials assembled using nanosheets for flexible and foldable energy storage devices, J. Energy Chem., 2020, 45, 67-73.

44 L. M. Cao, J. W. Wang, D. C. Zhong and T. B. Lu, Template-directed synthesis of sulphur doped NiCoFe layered double hydroxide porous nanosheets with enhanced electrocatalytic activity for oxygen evolution reaction, J. Mater. Chem. A, 2018, 6, 3224-3230.
45 N. Baliarsingh, L. Mohapatra and K. Parida, Design and development of a visible light harvesting $\mathrm{Ni}-\mathrm{Zn} / \mathrm{Cr}-\mathrm{CO}_{3}{ }^{2-}$ LDH system for hydrogen evolution, J. Mater. Chem. A, 2013, 1, 4236-4243.

46 K. Parida, M. Satpathy and L. Mohapatra, Incorporation of $\mathrm{Fe}^{3+}$ into $\mathrm{Mg} / \mathrm{Al}$ layered double hydroxide framework: effects on textural properties and photocatalytic activity for $\mathrm{H}_{2}$ generation, J. Mater. Chem. A, 2012, 22, 73507357.

47 F. Foerster and A. Piguet, Knowing the anodic oxygen evolution, Z. Elektrochem. Angew. Phys. Chem., 1904, 10, 714721.

48 H. N. Seiger and R. C. Shair, Oxygen evolution from heavily doped nickel oxide electrodes, J. Electrochem. Soc., 1961, 108, C163-C163.

49 R. Fayad, J. Dhainy, H. Ghandour and L. Halaoui, Electrochemical study of the promoting effect of $\mathrm{Fe}$ on oxygen evolution at thin 'NiFe-Bi'films and the inhibiting effect of Al in borate electrolyte, Catal. Sci. Technol., 2017, 7, 3876-3891.

50 M. Z. A. Munshi, A. C. C. Tseung and J. Parker, The dissolution of iron from the negative material in pocket plate nickel-cadmium batteries, J. Appl. Electrochem., 1985, 15, 711-717.

51 R. L. Tichenor, Nickel oxides-relation between electrochemical and foreign ion content, Ind. Eng. Chem., 1952, 44, 973-977.

52 N. Chandrasekaran and S. Muthusamy, Binderless, freestanding porous interconnects of $\mathrm{Ni}-\mathrm{Fe}$ alloy decorated reduced graphene oxide for oxygen evolution reaction, Langmuir, 2017, 33, 2-10.

53 S. Ci, S. Mao, Y. Hou, S. Cui, H. Kim, R. Ren, Z. Wen and J. Chen, Rational design of mesoporous NiFe-alloy-based hybrids for oxygen conversion electrocatalysis, J. Mater. Chem. A, 2015, 3, 7986-7993.

54 V. Vij, S. Sultan, A. M. Harzandi, A. Meena, J. N. Tiwari, W. G. Lee, T. Yoon and K. S. Kim, Nickel-based electrocatalysts for energy-related applications: oxygen reduction, oxygen evolution, and hydrogen evolution reactions, ACS Catal., 2017, 7, 7196-7225.

55 C. Liang, P. Zou, A. Nairan, Y. Zhang, J. Liu, K. Liu, S. Hu, F. Kang, H. J. Fan and C. Yang, Exceptional performance of hierarchical Ni-Fe oxyhydroxide@NiFe alloy nanowire array electrocatalysts for large current density water splitting, Energy Environ. Sci., 2020, 13, 86-95.

56 Y. Chen, K. Rui, J. Zhu, S. X. Dou and W. Sun, Recent progress on nickel-based oxide/(oxy) hydroxide electrocatalysts for the oxygen evolution reaction, Chem. - Eur. J., 2019, 25, 703-713.

57 D. Huang, J. Maa, L. Yu, D. Wu, K. Wang, M. Yang, D. Papoulis and S. Komarneni, $\mathrm{AgCl}$ and BiOCl composited with NiFe-LDH for enhanced photo-degradation of Rhodamine B, Sep. Purif. Technol., 2015, 156, 789-794.

58 R. Subbaraman, D. Tripkovic, K.-C. Chang, D. Strmcnik, A. P. Paulikas, P. Hirunsit, M. Chan, J. Greeley, V. Stamenkovic and N. M. Markovic, Trends in activity for 
the water electrolyser reactions on $3 \mathrm{~d} \mathrm{M}(\mathrm{Ni}, \mathrm{Co}, \mathrm{Fe}, \mathrm{Mn})$ hydr(oxy)oxide catalysts, Nat. Mater., 2012, 11, 550-557.

$59 \mathrm{~J}$. Mohammed-Ibrahim, A review on NiFe-based electrocatalysts for efficient alkaline oxygen evolution reaction, J. Power Sources, 2020, 448, 227375-227424.

60 Y. Hou, Z. Wen, S. Cui, X. Feng and J. Chen, Strongly coupled ternary hybrid aerogels of $\mathrm{N}$-deficient porous graphitic- $\mathrm{C}_{3} \mathrm{~N}_{4}$ nanosheets/N-doped graphene/NiFe-layered double hydroxide for solar-driven photoelectrochemical water oxidation, Nano Lett., 2016, 16, 2268-2277.

61 Z. Xue, X. Zhang, J. Qin and R. Liu, Revealing Ni-based layered double hydroxides as high-efficiency electrocatalysts for the oxygen evolution reaction: a DFT study, J. Mater. Chem. A, 2019, 7, 23091-23097.

62 S. Nayak and K. M. Parida, Dynamics of charge-transfer behavior in a plasmon-induced quasi-type-II p-n/n-n dual heterojunction in $\mathrm{Ag} @ \mathrm{Ag}_{3} \mathrm{PO}_{4} / \mathrm{g}-\mathrm{C}_{3} \mathrm{~N}_{4} / \mathrm{NiFe} \mathrm{LDH}$ nanocomposites for photocatalytic $\mathrm{Cr}(\mathrm{VI})$ reduction and phenol oxidation, ACS Omega, 2018, 3, 7324-7343.

63 S. Sirisomboonchai, S. Li, A. Yoshida, X. Li, C. Samart, A. Abudula and G. Guan, Fabrication of NiO microflake@ NiFe-LDH nanosheet heterostructure electrocatalysts for oxygen evolution reaction, ACS Sustainable Chem. Eng., 2018, 7, 2327-2334.

64 Z. Cai, X. Bu, P. Wang, J. C. Ho, J. Yang and X. Wang, Recent advances in layered double hydroxide electrocatalysts for the oxygen evolution reaction, J. Mater. Chem. A, 2019, 7, 5069-5089.

65 S. Yin, W. Tu, Y. Sheng, Y. Du, M. Kraft, A. Borgna and R. Xu, A highly efficient oxygen evolution catalyst consisting of interconnected Nickel-Iron-layered double hydroxide and carbon nanodomains, Adv. Mater., 2017, 30, 1705106-1705114.

66 X. Zhu, C. Tang, H.-F. Wang, Q. Zhang, C. Yang and F. Wei, Dual-sized NiFe layered double hydroxides in situ grown on oxygen-decorated self-dispersal nanocarbon as enhanced water oxidation catalysts, J. Mater. Chem. A, 2015, 3, 24540-24546.

67 C. Tang, H. F. Wang, X. L. Zhu, B. Q. Li and Q. Zhang, Advances in hybrid electrocatalysts for oxygen evolution reactions: rational integration of NiFe layered double hydroxides and nanocarbon, Part. Part. Syst. Charact., 2016, 33, 473-486.

68 X. Wang, J. Zhou, W. Xing, B. Liu, J. Zhang, H. Lin, H. Cui and S. Zhuo, Resorcinol-formaldehyde resin-based porous carbon spheres with high $\mathrm{CO}_{2}$ capture capacities, J. Energy Chem., 2017, 26, 1007-1013.

69 D.-C. Liu, L.-M. Cao, Z.-M. Luo, D.-C. Zhong, J.-B. Tan and T.-B. Lu, An in situ generated amorphous CoFePi and crystalline $\mathrm{Ni}\left(\mathrm{PO}_{3}\right)_{2}$ heterojunction as an efficient electrocatalyst for oxygen evolution, J. Mater. Chem. A, 2018, 6, 24920-24927.

70 M. Daud, M. S. Kamal, F. Shehzad and M. A. Al-Harthi, Advances in hybrid electrocatalysts for oxygen evolution reactions: rational integration of $\mathrm{NiFe}$ layered double hydroxides and nanocarbon, Carbon, 2016, 104, 241-252.
71 C. Li, M. Wei, D. G. Evans and X. Duan, Layered double hydroxide-based nanomaterials as highly efficient catalysts and adsorbents, Small, 2014, 26, 4469-4486.

72 T. Zhan, X. Liu, S. Lu and W. Hou, Nitrogen doped NiFe layered double hydroxide/reduced graphene oxide mesoporous nanosphere as an effective bifunctional electrocatalyst for oxygen reduction and evolution reactions, Appl. Catal., B, 2017, 205, 551-558.

73 J. Shen, P. Zhang, R. Xie, L. Chen, M. Li, J. Li, B. Ji, Z. Hu, J. Li, L. Song, Y. Wu and X. Zhao, Controlled selfassembled NiFe layered double hydroxides/reduced graphene oxide nanohybrids based on the solid-phase exfoliation strategy as an excellent electrocatalyst for the oxygen evolution reaction, ACS Appl. Mater. Interfaces, 2019, 11, 13545-13556.

74 J.-L. Shi, C. Tang, J.-Q. Huang, W. Zhu and Q. Zhang, Effective exposure of nitrogen heteroatoms in $3 \mathrm{D}$ porous graphene framework for oxygen reduction reaction and lithium-sulfur batteries, J. Energy Chem., 2018, 27, 167175.

75 Y. Gu, Y. Wang, W. An, Y. Men, Y. Rui, X. Fan and B. Li, A novel strategy to boost the oxygen evolution reaction activity of NiFe-LDHs with in situ synthesized 3D porous reduced graphene oxide matrix as both the substrate and electronic carrier, New J. Chem., 2019, 43, 6555-6562.

76 N. Manna, N. Ayasha, S. K. Singh and S. Kurungot, A NiFe layered double hydroxide-decorated N-doped entangledgraphene framework: a robust water oxidation electrocatalyst, Nanoscale Adv., 2020, 2, 1709-1717.

77 L. Cao, Y. Ma, A. Song, L. Bai, P. Zhang, X. Li and G. Shao, Stable composite of flower-like NiFe-layered double hydroxide nucleated on graphene oxide as an effective catalyst for oxygen reduction reaction, Int. J. Hydrogen Energy, 2019, 44, 5912-5920.

78 X. Yu, M. Zhang, W. Yuan and G. Shi, A high-performance three-dimensional Ni-Fe layered double hydroxide/graphene electrode for water oxidation, J. Mater. Chem. A, 2015, 3, 6921-6928.

79 X. Xu, H. Chu, Z. Zhang, P. Dong, R. Baines, P. M. Ajayan, J. Shen and M. Ye, Integrated energy aerogel of N, S-rGO/ $\mathrm{WSe}_{2} / \mathrm{NiFe}-\mathrm{LDH}$ for both energy conversion and storage, ACS Appl. Mater. Interfaces, 2017, 9, 32756-32766.

80 Y. Zheng, B. Cheng, W. You, J. Yu and W. Ho, 3D hierarchical graphene oxide-NiFe LDH composite with enhanced adsorption affinity to congo red, methyl orange and $\mathrm{Cr}(\mathrm{VI})$ ions, J. Hazard. Mater., 2019, 369, 214-225.

81 Y.-X. Zhu, M.-Y. Jiang, M. Liu, L.-K. Wu, G.-Y. Hou and Y.-P. Tang, Fe-V@NiO heterostructure electrocatalyst towards oxygen evolution reaction, Nanoscale, 2020, 12, 3803-3811.

82 D. Lim, S. Kim, N. Kim, E. Oh, S. E. Shim and S.-H. Baeck, Strongly coupled $\mathrm{Ni} / \mathrm{Ni}(\mathrm{OH})_{2}$ hybrid nanocomposites as highly active bifunctional electrocatalysts for overall water splitting, ACS Sustainable Chem. Eng., 2020, 8, 4431-4439.

83 W. Ma, R. Ma, C. Wang, J. Liang, X. Liu, K. Zhou and T. Sasaki, A superlattice of alternately stacked Ni-Fe 
hydroxide nanosheets and graphene for efficient splitting of water, ACS Nano, 2015, 9, 1977-1984.

84 C. Zhang, M. Shao, L. Zhou, Z. Li, K. Xiao and M. Wei, Hierarchical NiFe layered double hydroxide hollow microspheres with highly-efficient behavior toward oxygen evolution reaction, ACS Appl. Mater. Interfaces, 2016, 8, 3369733703.

85 L. Yu, J. F. Yang, B. Y. Guan, Y. Lu and X. W. D. Lou, Hierarchical hollow nanoprisms based on ultrathin Ni-Fe layered double hydroxide nanosheets with enhanced electrocatalytic activity towards oxygen evolution, Angew. Chem., Int. Ed., 2018, 57, 172-176.

86 Z. Li, M. Shao, H. An, Z. Wang, S. Xu, M. Wei, D. G. Evans and X. Duan, Fast electrosynthesis of Fe-containing layered double hydroxides arrays toward highly efficient electrocatalytic oxidation reactions, Chem. Sci., 2015, 6, 6624-6631.

87 F. Ning, M. Shao, S. Xu, Y. Fu, R. Zhang, M. Wei, D. G. Evans and X. Duan, TiO 2/graphene/NiFe-layered double hydroxide nanorod array photoanodes for efficient photoelectrochemical water splitting, Energy Environ. Sci., 2016, 9, 2633-2643.

88 X. Han, Y. Wei, J. Su and Y. Zhao, Low-cost oriented hierarchical growth of $\mathrm{BiVO}_{4} / \mathrm{rGO} / \mathrm{NiFe}$ nanoarrays photoanode for photoelectrochemical water splitting, ACS Sustainable Chem. Eng., 2018, 6, 14695-14703.

89 X. Dong, J. Huang, H. Wan, F. Chen, Y. Lin, X. Xu, R. Ma and T. Sasaki, Recent progress in functionalized layered double hydroxides and their application in efficient electrocatalytic water oxidation, J. Energy Chem., 2019, 32, 93-104.

90 L.-M. Cao, Y.-W. Hu, D.-C. Zhong and T.-B. Lu, Templatedirected growth of bimetallic prussian blue analogue nanosheet arrays and their derived porous metal oxide for oxygen evolution reaction, ChemSusChem, 2018, 11, 37083713.

91 L.-M. Cao, D. Lu, D.-C. Zhong and T.-B. Lu, Prussian blue analogues and their derived nanomaterials for electrocatalytic water splitting, Coord. Chem. Rev., 2020, 407, 213156-213173.

92 Y. Jia, L. Zhang, G. Gao, H. Chen, B. Wang, J. Zhou, M. T. Soo, M. Hong, X. Yan, G. Qian, J. Zou, A. Du and $\mathrm{X}$. Yao, A heterostructure coupling of exfoliated Ni-Fe hydroxide nanosheet and defective graphene as a bifunctional electrocatalyst for overall water splitting, $A d v$. Mater., 2017, 29, 1700017-1700024.

93 T. Zhan, Y. Zhang, X. Liu, S. Lu and W. Hou, NiFe layered double hydroxide/reduced graphene oxide nanohybrid as an efficient bifunctional electrocatalyst for oxygen evolution and reduction reactions, J. Power Sources, 2016, 333, 53-60.

94 Y. Cao, G. Li and X. Li, Graphene/layered double hydroxide nanocomposite: properties, synthesis, and applications, Chem. Eng. J., 2016, 292, 207-223.

95 X. Long, Z. Wang, S. Xiao, Y. An and S. Yang, Transition metal based layered double hydroxides tailored for energy conversion and storage, Mater. Today, 2016, 19, 213-226.
96 Y. Jiang, Y. Song, Y. Li, W. Tian, Z. Pan, P. Yang, Y. Li, Q. $\mathrm{Gu}$ and $\mathrm{L}$. $\mathrm{Hu}$, Charge transfer in ultrafine $\mathrm{LDH}$ nanosheets/graphene interface with superior capacitive energy storage performance, ACS Appl. Mater. Interfaces, 2017, 9, 37645-37654.

97 Q. Wang and D. O'Hare, Recent advances in the synthesis and application of layered double hydroxide (LDH) nanosheets, Chem. Rev., 2012, 112, 4124-4155.

98 Y. Wang, D. Yan, S. EI Hankari, Y. Zou and S. Wang, Recent progress on layered double hydroxides and their derivatives for electrocatalytic water splitting, Adv. Sci., 2018, 5, 1800064-1800095.

99 D. H. Youn, Y. B. Park, J. Y. Kim, G. Magesh, Y. J. Jang and J. S. Lee, One-pot synthesis of NiFe layered double hydroxide/reduced graphene oxide composite as an efficient electrocatalyst for electrochemical and photoelectrochemical water oxidation, J. Power Sources, 2015, 294, 437-443.

100 D. C. Xia, L. Zhou, S. Qiao, Y. Zhang, D. Tang, J. Liu, H. Huang, Y. Liu and Z. Kang, Graphene/Ni-Fe layered double-hydroxide composite as highly active electrocatalyst for water oxidation, Mater. Res. Bull., 2016, 74, 441446.

101 R. Li, J. Xu, Q. Pan, J. Ba, T. Tang and W. Luo, One-step synthesis of NiFe layered double hydroxide nanosheet array/N-doped graphite foam electrodes for oxygen evolution reactions, ChemistryOpen, 2019, 8, 1027-1032.

102 X. Wang, G. Sun, P. Routh, D. H. Kim, W. Huang and P. Chen, Heteroatom-doped graphene materials: syntheses, properties and applications, Chem. Soc. Rev., 2014, 43, 7067-7098.

103 Y. Zhang, J. Ge, L. Wang, D. Wang, F. Ding, X. Tao and W. Chen, Manageable N-doped graphene for high performance oxygen reduction reaction, Sci. Rep., 2013, 3, 2771-2778.

104 R. Li, J. Xu, Q. Pan, J. Ba, T. Tang and W. Luo, One-step synthesis of NiFe layered double hydroxide nanosheet array/N-doped graphite foam electrodes for oxygen evolution reactions, ChemistryOpen, 2019, 8, 1027-1032.

105 D. Zhou, Z. Cai, X. Lei, W. Tian, Y. Bi, Y. Jia, N. Han, T. Gao, Q. Zhang, Y. Kuang, J. Pan, X. Sun and X. Duan, NiCoFe-layered double hydroxides/N-doped graphene oxide array colloid composite as an efficient bifunctional catalyst for oxygen electrocatalytic reactions, Adv. Energy Mater., 2017, 8, 1701905-1701911.

106 C. Tang, H. S. Wang, H. F. Wang, Q. Zhang, G. L. Tian, J. Q. Nie and F. Wei, Spatially confined hybridization of nanometer-sized NiFe hydroxides into nitrogen-doped graphene frameworks leading to superior oxygen evolution reactivity, Adv. Mater., 2015, 27, 4516-4522.

107 J. Zhu, X. Yang, Z. Fu, J. He, C. Wang, W. Wu and L. Zhang, Three-dimensional macroassembly of sandwich-like, hierarchical, porous carbon/graphene nanosheets towards ultralight, superhigh surface area, multifunctional aerogels, Chem. - Eur. J., 2016, 22, 25152524 . 
108 Y. Yang, R. Zhao, T. Zhang, K. Zhao, P. Xiao, Y. Ma, P. M. Ajayan, G. Shi and Y. Chen, Graphene-based standalone solar energy converter for water desalination and purification, ACS Nano, 2018, 12, 829-835.

109 X. Wang, Y. Yang, L. Diao, Y. Tang, F. He, E. Liu, C. He, C. Shi, J. Li, J. Sha, S. Ji, P. Zhang, L. Ma and N. Zhao, CeOx-decorated NiFe-layered double hydroxide for efficient alkaline hydrogen evolution by oxygen vacancy engineering, ACS Appl. Mater. Interfaces, 2018, 10, 3514535153.

110 P. Xiong, X. Zhang, H. Wan, S. Wang, Y. Zhao, J. Zhang, D. Zhou, W. Gao, R. Ma, T. Sasaki and G. Wang, Interface modulation of two-dimensional superlattices for efficient overall water splitting, Nano Lett., 2019, 19, 4518-4526.

111 Y. Han, P. Li, J. Liu, S. Wu, Y. Ye, Z. Tian and C. Liang, Strong $\mathrm{Fe}^{3+}-\mathrm{O}(\mathrm{H})-\mathrm{Pt}$ interfacial interaction induced excellent stability of $\mathrm{Pt} / \mathrm{NiFe}-\mathrm{LDH} / \mathrm{rGO}$ electrocatalysts, Sci. Rep., 2018, 22, 1-9.

112 X. Long, J. Li, S. Xiao, K. Yan, Z. Wang, H. Chen and S. Yang, A strongly coupled graphene and FeNi double hydroxide hybrid as an excellent electrocatalyst for the oxygen evolution reaction, Angew. Chem., Int. Ed., 2014, 53, 7584-7588.

113 W. S. Hummers and R. E. Offeman, Preparation of graphitic oxide, J. Am. Chem. Soc., 1958, 80, 1339-1339.

114 X. Dong, L. Wang, D. Wang, C. Li and J. Jin, Layer-by-layer engineered Co-Al hydroxide nanosheets/graphene multilayer films as flexible electrode for supercapacitor, Langmuir, 2012, 28, 293-298.

115 P. Xiong, R. Ma, N. Sakai, L. Nurdiwijayanto and T. Sasaki, A unilamellar metallic $\mathrm{MoS}_{2}$ /graphene superlattice for efficient sodium storage and hydrogen evolution, ACS Energy Lett., 2018, 4, 997-1005.

116 D. C. Xia, L. Zhou, S. Qiao, Y. Zhang, D. Tang, J. Liu, H. Huang, Y. Liu and Z. Kang, Graphene/Ni-Fe layered double-hydroxide composite as highly active electrocatalyst for water oxidation, Mater. Res. Bull., 2016, 74, 441446.

117 Y. Li, M. Zhao, Y. Zhao, L. Song and Z. Zhang, FeNi layered double-hydroxide nanosheets on a 3D carbon network as an efficient electrocatalyst for the oxygen evolution reaction, Part. Part. Syst. Charact., 2016, 33, 158-166.

118 I. Vincent and D. Bessarabov, Low cost hydrogen production by anion exchange membrane electrolysis, Renewable Sustainable Energy Rev., 2018, 81, 1690-1704.

119 C. C. McCrory, S. Jung, I. M. Ferrer, S. M. Chatman, J. C. Peters and T. F. Jaramillo, Benchmarking hydrogen evolving reaction and oxygen evolving reaction electrocatalysts for solar water splitting devices, J. Am. Chem. Soc., 2015, 137, 4347-4357.

120 H. Wan, X. Liu, H. Wang, R. Ma and T. Sasaki, Recent advances in developing high-performance nanostructured electrocatalysts based on 3d transition metal elements, Nanoscale Horiz., 2019, 4, 789-808.

121 S. L. Candelaria, N. M. Bedford, T. J. Woehl, N. S. Rentz, A. R. Showalter, S. Pylypenko, B. A. Bunker, S. Lee,
B. Reinhart and Y. Ren, Multi-component Fe-Ni hydroxide nanocatalysts for oxygen evolution and methanol oxidation reactions under alkaline conditions, ACS Catal., 2017, 7, 365-379.

122 W. Zhang, Y. Wu, J. Qi, M. Chen and R. Cao, Electrocatalysis: a thin NiFe hydroxide film formed by stepwise electrodeposition strategy with significantly improved catalytic water oxidation efficiency, Adv. Energy Mater., 2017, 7, 1602547-1602552.

123 J. R. Swierk, S. Klaus, L. Trotochaud, A. T. Bell and T. D. Tilley, Electrochemical study of the energetics of the oxygen evolution reaction at nickel iron (oxy) hydroxide catalysts, J. Phys. Chem. C, 2015, 119, 19022-19029.

124 D. Friebel, M. W. Louie, M. Bajdich, K. E. Sanwald, Y. Cai, A. M. Wise, M. J. Cheng, D. Sokaras, T. C. Weng, R. Alonso-Mori, R. C. Davis, J. R. Bargar, J. K. Nørskov, A. Nilsson and A. T. Bell, Identification of highly active Fe sites in (Ni, Fe) $\mathrm{OOH}$ for electrocatalytic water splitting, J. Am. Chem. Soc., 2015, 137, 1305-1313.

125 F. Song and X. Hu, Exfoliation of layered double hydroxides for enhanced oxygen evolution catalysis, Nat. Commun., 2014, 5, 1-9.

126 N. Han, F. Zhao and Y. Li, Ultrathin nickel-iron layered double hydroxide nanosheets intercalated with molybdate anions for electrocatalytic water oxidation, J. Mater. Chem. A, 2015, 3, 16348-16353.

127 W. Ma, R. Ma, J. Wu, P. Sun, X. Liu, K. Zhou and T. Sasaki, Development of efficient electrocatalysts via molecular hybridization of NiMn layered double hydroxide nanosheets and graphene, Nanoscale, 2016, 8, 1042510432.

128 Y. Jia, L. Zhang, A. Du, G. Gao, J. Chen, X. Yan, C. L. Brown and X. Yao, Defect graphene as a trifunctional catalyst for electrochemical reactions, Adv. Mater., 2016, 28, 9532-9538.

129 T. Liu, Y. F. Guo, Y. M. Yan, F. Wang, C. Deng, D. Rooney and K. N. Sun, CoO nanoparticles embedded in threedimensional nitrogen/sulfur co-doped carbon nanofiber networks as a bifunctional catalyst for oxygen reduction/ evolution reactions, Carbon, 2016, 106, 84-92.

130 Y. Hou, M. R. Lohe, J. Zhang, S. Liu, X. Zhuang and $\mathrm{X}$. Feng, Vertically oriented cobalt selenide/NiFe layereddouble-hydroxide nanosheets supported on exfoliated graphene foil: an efficient 3D electrode for overall water splitting, Energy Environ. Sci., 2016, 9, 478-483.

131 C. H. Choi, M. Kim, H. C. Kwon, S. J. Cho, S. Yun, H.-T. Kim, K. J. Mayrhofer, H. Kim and M. Choi, Tuning selectivity of electrochemical reactions by atomically dispersed platinum catalyst, Nat. Commun., 2016, 7, 1-9.

132 J. Duan, S. Chen, B. A. Chambers, G. G. Andersson and S. Z. Qiao, 3D $\mathrm{WS}_{2}$ nanolayers@ heteroatom-doped graphene films as hydrogen evolution catalyst electrodes, Adv. Mater., 2015, 27, 4234-4241.

133 G. Wu, K. L. More, C. M. Johnston and P. Zelenay, Highperformance electrocatalysts for oxygen reduction derived 
from polyaniline, iron, and cobalt, Science, 2011, 332, 443-447.

134 L. Sun, J. Sun, X. Yang, S. Bai, Y. Feng, R. Luo, D. Li and A. Chen, An integrating photoanode consisting of $\mathrm{BiVO}_{4}$, rGO and LDH for photoelectrochemical water splitting, Dalton Trans., 2019, 48, 16091-16098.

135 A. Fujishima and K. Honda, Photolysis-decomposition of water at the surface of an irradiated semiconductor, Nature, 1972, 238, 37-38.

136 J. M. Lehn and R. Ziessel, Photochemical generation of carbon monoxide and hydrogen by reduction of carbon dioxide and water under visible light irradiation, Proc. Natl. Acad. Sci. U. S. A., 1982, 79, 701-704.

137 S. Sato and J. M. White, Photocatalytic production of hydrogen from water and Texas lignite by use of a platinized titania catalyst, Ind. Eng. Chem. Prod. Res. Dev., 1980, 4, 542-544.

138 K. Domen, S. Naito, M. Soma, T. Onishi and K. Tamaru, Photocatalytic decomposition of water vapour on an NiO$\mathrm{SrTiO}_{3}$ catalyst, J. Chem. Soc., Chem. Commun., 1980, 543544.

139 X. Chen, S. Shen, L. Guo and S. S. Mao, Semiconductorbased photocatalytic hydrogen generation, Chem. Rev., 2010, 110, 6503-6570.

140 L. Mohapatra and K. Parida, A review on the recent progress, challenges and perspective of layered double hydroxides as promising photocatalysts, J. Mater. Chem. A, 2016, 4, 10744-10766.

141 D. P. Sahoo, S. Patnaik and K. Parida, Construction of a $\mathrm{Z}$-scheme dictated $\mathrm{WO}_{3}-\mathrm{X} / \mathrm{Ag} / \mathrm{ZnCr} \mathrm{LDH}$ synergistically visible light-induced photocatalyst towards tetracycline degradation and $\mathrm{H}_{2}$ evolution, ACS Omega, 2019, 4, 14721-14741.

142 D. Kandi, D. P. Sahoo, S. Martha and K. Parida, Rational design of a coupled confronting Z-scheme system toward photocatalytic refractory pollutant degradation and water splitting reaction, Adv. Mater. Interfaces, 2019, 6, 1900370.

143 L. Mohapatra and K. Parida, Recent progress in the development of carbonate-intercalated $\mathrm{Zn} / \mathrm{Cr} \mathrm{LDH}$ as a novel photocatalyst for hydrogen evolution aimed at the utilization of solar light, Dalton Trans., 2012, 41, 1173-1178.

144 L. Mohapatra and K. Parida, Dramatic activities of vanadate intercalated bismuth doped LDH for solar light photocatalysis, Phys. Chem. Chem. Phys., 2014, 16, 1698516996.

145 L. Mohapatra, D. Patra, K. Parida and S. J. Zaidi, Enhanced photocatalytic activity of a molybdate-intercalated iron-based layered double hydroxide, Eur. J. Inorg. Chem., 2017, 3, 723-733.

146 L. Mohapatra and K. Parida, A review of solar and visible light active oxo-bridged materials for energy and environment, Catal. Sci. Technol., 2017, 7, 2153-2164. 